\title{
Magnetic resonance imaging investigations of directed influence in the brain: methodological contributions and application to task switching studies
}

Citation for published version (APA):

Roebroeck, A. F. (2006). Magnetic resonance imaging investigations of directed influence in the brain: methodological contributions and application to task switching studies. [Doctoral Thesis, Maastricht University]. Datawyse / Universitaire Pers Maastricht. https://doi.org/10.26481/dis.20061123ar

Document status and date:

Published: 01/01/2006

DOI:

10.26481/dis.20061123ar

Document Version:

Publisher's PDF, also known as Version of record

Please check the document version of this publication:

- A submitted manuscript is the version of the article upon submission and before peer-review. There can be important differences between the submitted version and the official published version of record. People interested in the research are advised to contact the author for the final version of the publication, or visit the DOI to the publisher's website.

- The final author version and the galley proof are versions of the publication after peer review.

- The final published version features the final layout of the paper including the volume, issue and page numbers.

Link to publication

\footnotetext{
General rights rights.

- You may freely distribute the URL identifying the publication in the public portal. please follow below link for the End User Agreement:

www.umlib.nl/taverne-license

Take down policy

If you believe that this document breaches copyright please contact us at:

repository@maastrichtuniversity.nl

providing details and we will investigate your claim.
}

Copyright and moral rights for the publications made accessible in the public portal are retained by the authors and/or other copyright owners and it is a condition of accessing publications that users recognise and abide by the legal requirements associated with these

- Users may download and print one copy of any publication from the public portal for the purpose of private study or research.

- You may not further distribute the material or use it for any profit-making activity or commercial gain

If the publication is distributed under the terms of Article 25fa of the Dutch Copyright Act, indicated by the "Taverne" license above, 


\section{Magnetic resonance imaging investigations of}

\section{directed influence in the brain}

\section{methodological contributions and} application to task switching studies

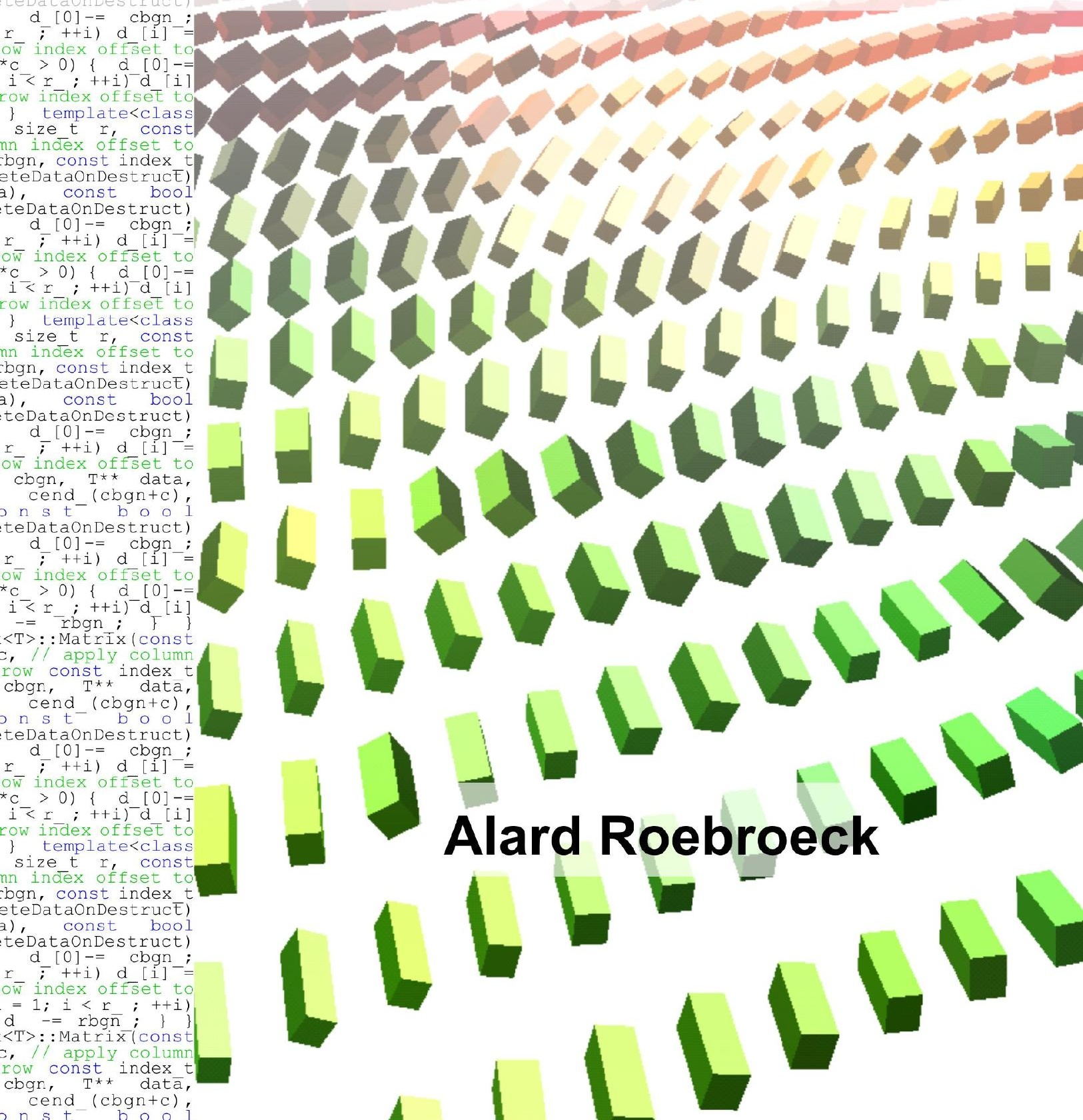

eteDataOnDestrucE) tedataonDestruct) $d_{-}[0]-=$ cbgn eteDataOnDestrucE) const bool r $\bar{i}++i)$ d $[i]-^{\prime}$ cbgn, $T^{\star \star}$ data, $\left.-{ }^{-}+i\right)$ d $[i]-$ a $\{$ [0]-= $-=$ rbon. $\xi^{\text {r }}$ $\langle\mathrm{T}\rangle$ : : Matrix (const row const index $t$ cbgr, $T^{* \star}$ data, $n \mathrm{~s} t$ (c) teDataOnDestruct) $\left.r_{;}{ }^{d}++i\right)$ d $[i]-=$ windex ofiset $\left.{ }^{*} \mathrm{C}<\mathrm{r} ; \quad ;++\mathrm{i}\right)-\mathrm{d}[\mathrm{i}]$ m index offset to etedataOnDestruct) a), const bool edataondestruct) r $\bar{i}++i)$ d $[i]-$ windex oftset $\mathrm{d}-=\operatorname{rbg} \bar{n} ;\}$
$\langle\mathrm{T}\rangle$ i/ apoly column cbgr, $\mathrm{T}^{\star \star}$ datá, cend_ $(\mathrm{cbg} n+\mathrm{c})$ 


\section{Magnetic resonance imaging investigations of directed influence in the brain}

methodological contributions and application

to task switching studies

Alard Roebroeck 
Colophon

Cover design: $\quad$ Alard Roebroeck \& Ellen Dreezens

Layout:

Ellen Dreezens \& Alard Roebroeck

Production: $\quad$ Datawyse | Universitaire pers Maastricht

(C) Alard Roebroeck, Maastricht, 2006

ISBN-10: 90-5278-584-8

ISBN-13: 978-90-5278-584-4

The work in this thesis was supported by the Human Frontier Science Program and was conducted at Maastricht University 


\title{
Magnetic resonance imaging investigations of directed influence in the brain
}

\author{
methodological contributions and application \\ to task switching studies
}

\section{PROEFSCHRIFT}

Ter verkrijging van de graad van doctor aan de Universiteit Maastricht, op gezag van de Rector Magnificus, Prof. Mr. G. P. M. F. Mols volgens het besluit van het College van Decanen, in het openbaar te verdedigen op donderdag 23 november 2006 om 14.00 uur

door

Alard Franc Roebroeck

Geboren op 28 juni 1978 te Reuver 
Promotor:

Prof. Dr. R. W. Goebel

Copromotor:

Dr. E. Formisano

Beoordelingscommissie:

Prof. Dr. E. O. Postma

(Voorzitter)

Dr. B. Horwitz

(National Institutes of Health, Bethesda, USA)

Dr. D-S. Kim

(Boston University School of Medicine, Boston, USA)

Prof Dr. F Di Salle

Dr. A. Sack 


\section{Contents}

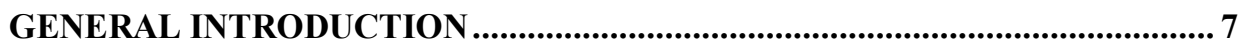

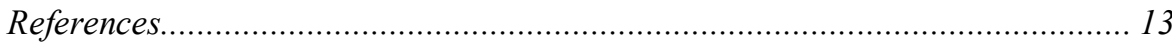

CHAPTER 1: MAPPING DIRECTED INFLUENCE OVER THE BRAIN USING

GRANGER CAUSALITY AND FMRI ................................................................ 15

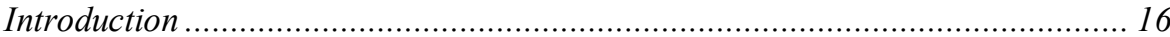

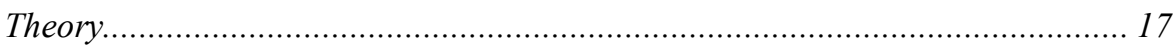

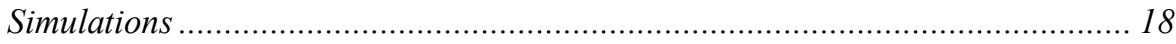

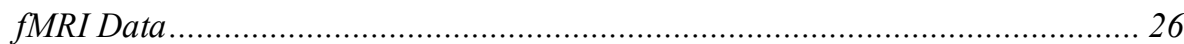

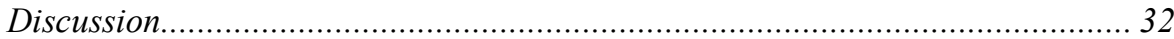

Appendix A: Computation of the influence measures .......................................... 36

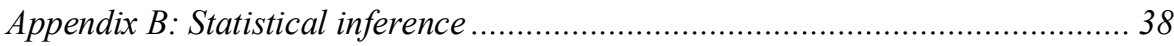

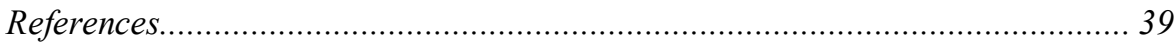

CHAPTER 2: THE INTERACTIONS OF PARIETAL AND PREMOTOR

CORTEX DURING TASK SWITCHING.......................................................43

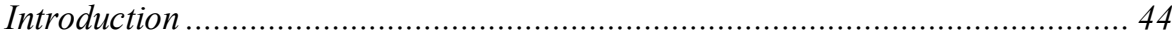

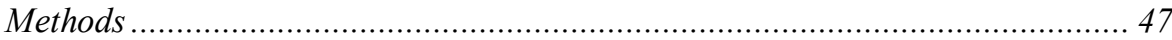

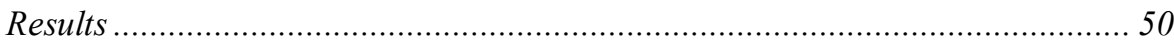

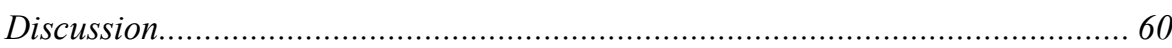

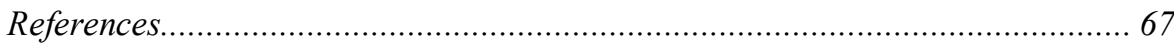

CHAPTER 3: HIGH RESOLUTION DIFFUSION TENSOR IMAGING AND

TRACTOGRAPHY OF THE HUMAN OPTIC CHIASM AT 9.4T........................ 69

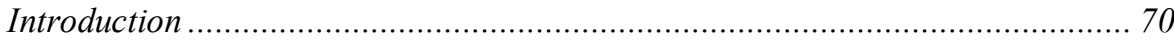

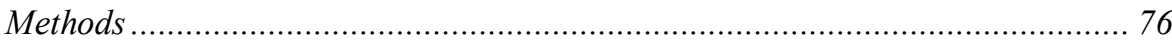

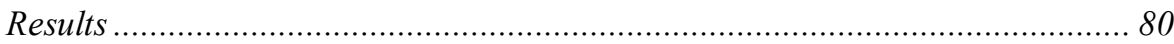

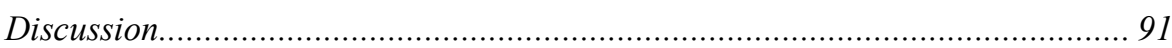

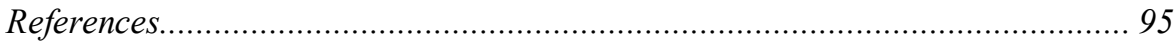

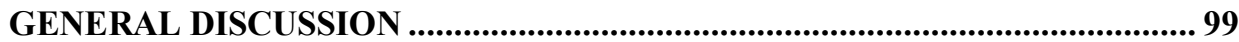

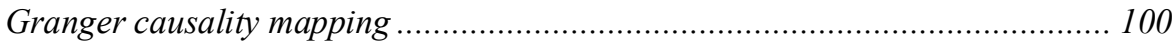

Diffusion tensor imaging and fiber tracking .................................................. 104

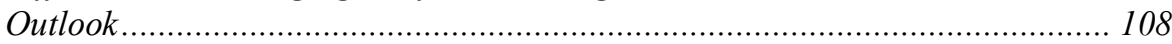

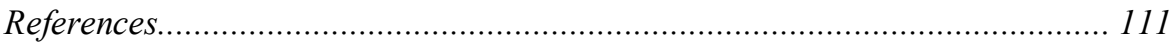

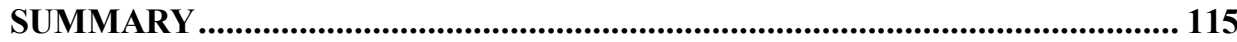

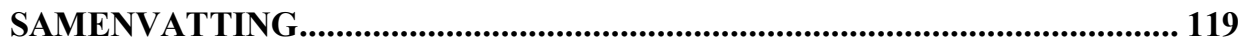

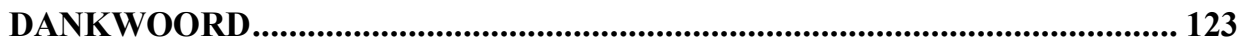

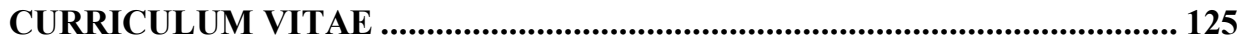


General Introduction 
In the past few decades, the endeavor of the field of cognitive neuroscience has been to unravel the intricate neuronal processes in the brain that underlie perception, cognition and behavior. Its continuing aim is to link the language of cognitive psychology to that of neuroscience, by identifying the neuronal substrates of cognitive component processes such as perception, attention, memory, motor planning and executive functions in their various forms. This study was revolutionized by the advent of techniques capable of measuring whole-brain activity non-invasively, in such a way that it can be recorded at the neuronal population scale during the performance of perceptual, cognitive, language, and motor tasks.

The two main classes of methods or modalities are the electrophysiological methods, electro-encephalography (EEG) and magneto-encephalography (MEG), and the hemodynamic/metabolic methods, positron emission tomography (PET) and functional magnetic resonance imaging (fMRI). The electrophysiological methods measure the small electrical and magnetic fields resulting from synchronous firing of large neuronal populations with sensors at the scalp. Although the temporal resolution of these measurements are very high (in the order of milliseconds), the localization of the activity recorded at the scalp to its source in the cortex is an ill-defined inverse problem, resulting in low spatial resolution (in the order of centimeters). Additionally, since the sensitivity of the sensors falls off rapidly with distance, the electrophysiological methods are virtually incapable of recording activity in deep brain-sources such as the thalamus, basal ganglia, and the brainstem. The hemodynamic/metabolic methods measure localized changes in capillary blood flow, blood volume, and metabolite concentrations resulting from neuronal activity. Although the spatial resolution of these methods is high, particularly in fMRI (in the order of millimeters), the time needed to acquire a high resolution volume with considerable spatial coverage limits the temporal resolution (in the order of seconds). Additionally, the indirect measurement of neuronal activity through hemodynamic and metabolic mechanisms further limits the achievable temporal resolution and makes the exact aspect of neural activity that is measured (synaptic activity, graded post-synaptic potentials, or axonal spikes) less clear than for other methods. fMRI is the technique used in this thesis to study functional brain activity, because of its favorable (and adjustable) trade-off between spatial and temporal resolution.

\section{Functional specialization and functional integration}

Numerous investigations using both classes of methods have led to the localization of neural activity tied to perceptual, cognitive, language and motor processes, both in time (primarily through the use of electrophysiological methods) and in space (primarily through the use of hemodynamic/metabolic methods). This process of (human) brain mapping, where each cortical area or subcortical structure is mapped to a discrete function or sub-process that it performs at a discrete time (and vice versa), has become one of the cornerstones of the field. This methodology of functional localization and functional specialization is more than just a modern version of phrenology. The reliable and repeated localization of certain cognitive sub-processes to discrete cortical areas and subcortical structures, and the specification of the specialized computational processes performed in neuronal populations is of great value to our understanding of the workings of the brain. Nonetheless, functional specialization is only part of the story. 
None of the specialized computational modules in the brain is likely to perform its task in isolation. Rather, it receives inputs from other regions, sends its own outputs to yet other neural structures, and its processing might be modulated by further cortical areas or subcortical structures. Thus, each neuronal population in the brain performs its tasks in continuous interaction with other areas. An important part of what a neural structure does is determined not only by its own local processing, but also in how it interacts with other systems. In this respect, the fundamental unit in the functioning brain might not be the discrete cortical area or sub-cortical structure, but the large-scale network of neural structures performing a fundamental computational task together in close interaction. This is the functional integration view on brain function (Friston, 1994, 2002).

\section{Connectivity}

The interaction of spatially discrete neuronal system requires connectivity. A large-scale network of neuronal structures can form an integrated network of interacting components only if those components are connected by physical communication channels and if those channels are used for interaction in the computational task under investigation. In the context of such large-scale interaction between discrete brain systems, three kinds of connectivity can be operationally distinguished: anatomical connectivity, functional connectivity, and effective connectivity. Anatomical or structural connectivity refers to the existence of a direct axonal connection between two structures. Anatomical connectivity is traditionally investigated by invasive methods such as molecular tracer studies and post-mortem dissection. Recently, with the advent of diffusion weighted magnetic resonance imaging and diffusion tensor imaging (DTI), new possibilities have arisen to study inter-regional anatomical connectivity non-invasively in vivo. DTI is the magnetic resonance imaging technique that is used in this thesis to study anatomical brain connectivity.

Functional connectivity is defined as the temporal correlation between remote neurophysiological events (Friston, 1994). Functional connectivity exists between two neural populations if a correlation (more generally: a statistical dependence or mutual information) is found between activity signals simultaneously recorded from the two populations. It is task-relative, i.e. functional connectivity between two neural systems present during the performance of one task, does not need to be present during different task or computation. Functional connectivity can be estimated on signals from different measurement methods and can be quantified with different computational measures, such as correlation (in the time domain), coherence (in the frequency domain), or multivariate signal decomposition methods such as principle components analysis (PCA) and independent component analysis (ICA). Generally, there are relatively few operational assumptions involved in the estimation of functional connectivity by one of the above methods, which makes them easy to compute and use. However, the existence of functional connectivity per se between two neuronal populations does not allow any statements about the underlying causal mechanisms. A correlation does not tell us exactly how the populations influenced each other's processing to perform an important computation together. Functional connectivity can exist because a population sends input to another population, because of the reverse flow of information, or because of a bidirectional communication. Moreover, functional connectivity can exist 
when the interaction of the cortical regions is mediated by a third structure, or when both receive input from a common source without interacting themselves.

Models that incorporate the concept of effective connectivity aim at characterizing the true causal computational mechanisms underlying the observed patterned of functional connectivities. Effective connectivity is defined as the influence exerted from one neuronal population on another (Friston, 1994). Effective connectivity was originally defined specifically with respect to data obtained with PET and fMRI and, like functional connectivity, is task-relative. The concrete application of this definition to measured data requires further specification, in the form of supporting assumptions, of what 'influence' means in terms of the data. These assumptions are incorporated into models, whose parameters (quantifying effective connectivity) are to be estimated on the acquired data. Thus, any operational version of an effective connectivity method is always modelrelative. For general effective connectivity models the assumptions can be grouped into two: the anatomical model and the mathematical model (Buchel and Friston, 2000; Buchel and Friston, 1997; McIntosh and Gonzalez-Lima, 1994). The anatomical or structural model determines which set of predefined areas are assumed to participate in the network underlying a cognitive task or processes under investigation. Furthermore, the assumed anatomical connections between these areas are specified. Thus the anatomical model can be represented as a directed graph model incorporating prior knowledge about participating structures and the connections between them. The mathematical model determines how activity measured in one region is a mathematical function of that in another when there is indeed a causal influence between the two. In other words, the mathematical model specifies how interaction at the neuronal population level is to be detected as statistical dependencies between measured signals. The mathematical model mostly takes the form of a system of equations that model the activity of each of the incorporated regions as a function of some selected others (determined by the anatomical model). The parameters in these equations, to be estimated from the data, directly or indirectly represent effective connectivity between discrete regions. Additionally, the mathematical model often provides for the modulation of the effective connectivity by experimental cognitive factors such as the presence of attention. Quite a few forms for the mathematical model have been explored in recent years such as regression equations (psycho-physiological interactions, Buchel and Friston, 1997), covariance structural equation models (Buchel and Friston, 1997; McIntosh and Gonzalez-Lima, 1994), nonlinear system identification procedures in the form of Volterra kernels (Friston and Buchel, 2000), multivariate autoregressive models (Harrison et al., 2003), and deterministic state-space models (Dynamic causal models, Friston et al., 2003). Without exception these methods require the strong assumptions present in an (implicit or explicit) anatomical model for their specification. In other words, these effective connectivity models are highly dependent on a pre-specified directedgraph model of the assumed connectivity between the involved regions.

The prior assumptions in the anatomical model are derived from a mixture of sources, such as known human (sub)cortical connectivity, molecular tracer studies in monkeys, and hypothesized theoretical network models. Often parts of the anatomical model are approximations or abstractions of the (unknown) true anatomical connectivity between systems. This points to the most serious problem of using pre-specified anatomical models: when the set of regions assumed to be relevant or the assumed 
anatomical connectivity between them is incomplete or incorrect, severe errors can enter into the effective connectivity inferences based on the model. The dynamics of activity in the included regions is generally mathematically modeled as a propagation or flow of activity through the assumed anatomical connections. All inferences on effective connectivity and its modulation by experimental factors is based on the fit of these modeled dynamics to the measured signal. Therefore, inaccuracies and bias can enter into these inferences when the anatomical model is wrong or incomplete.

\section{Research aims and overview}

This thesis investigates whether current effective connectivity methods can be complemented with alternative forms of analysis that relieve the strong dependence on an assumed anatomical model. Two such alternative forms of analysis are investigated: Granger causality mapping (GCM) and diffusion tensor imaging (DTI) based fiber tracking. Granger causality mapping (Goebel et al., 2003; Roebroeck et al., 2005), developed in this thesis, is an fMRI-based technique that maps effective connectivity for a selected reference region over the imaging volume. More precisely, all regions that are a source of influence to the reference region, and all regions that are a target of influence from the reference region are identified and mapped over the brain. The method is exploratory in nature and does not require the specification of a directed graph model of assumed anatomical connectivity. The concept of Granger causality is used as a mathematical model to specify how directed influence is defined and estimated in fMRI data. Granger causality quantifies the usefulness of unique information in one of the time series in predicting values of the other. Specifically, if incorporating past values of time series $\mathrm{x}$ improves the prediction of the current value of time series $\mathrm{y}$, we say that $\mathrm{x}$ Granger causes y. Thus, temporal precedence is used to identify the direction of causality from information in the data. GCM can be useful when the network of regions and connections underlying a specific task is not well known. Particularly in early phases of investigation of a particular cognitive process or task, it can be used to identify all participating interacting regions in a data-driven way.

In this thesis, GCM is applied to a study of switching visuomotor association tasks to illustrate its potential to identify interacting regions in a complex cognitive task. The tasks were designed to investigate and distinguish the different aspects of task switching in the context of arbitrary visuomotor associations. Task switching refers to human ability to flexibly change behavior according to contextual cues, particularly when the requirements of a task change abruptly. Very little is known about the network of interacting regions in the brain that underlies successful task switching. The investigation of task switching with GCM shows the important convergence of method, experimental design, and application that is central to the advancement of knowledge in cognitive neuroscience.

Diffusion tensor imaging and fiber tracking are techniques developed to assess anatomical connectivity in the human brain non-invasively in vivo (Basser et al., 1994; Mori et al., 1999). Diffusion tensor imaging (DTI) is a magnetic resonance imaging technique that can quantify the directional dependence of water diffusion in biological tissue. In brain white-matter tissue, water diffusion is greater along the direction of axonbundles than perpendicular to it. By quantifying the degree and direction of this diffusion anisotropy, DTI can be used to probe the micro-structure of white-matter fiber-bundles 
non-invasively in vivo. Fiber tracking or tractography refers to the derivation of area-toarea axonal connectivity from the properties of local diffusion profiles. Many promising early results have been obtained using this technique, such as the reliable identification of many of the large white matter structures such as the cortical association tracts in individual subjects. However, DTI tractography is known to have limitations primarily when complex fiber-architectures (such as sharp curves and crossings) are averaged into relatively large voxels. This can lead to misrepresentation of the local diffusion profile and errors (false positives and false negatives) in the fiber tracking results. This thesis considers DTI tractography as a technique that could yield reliable knowledge on the true individual anatomical connectivity of structures and cortical regions in the brain.

The main chapters in this thesis can be read in isolation. They correspond to research articles that either have been published (chapter 1), or are in preparation (chapters 2 \& 3). Each has its own abstract, introduction and discussion. The general conclusion must be viewed in relation to the main chapters.

In chapter 1, fMRI-based Granger causality mapping (GCM) is introduced as a datadriven way of mapping directed influence over the brain. The technique is validated using computational simulations and is applied to fMRI data. The soundness of statistical inferences on effective connectivity based on GCM is verified and the effects of the strength and time delay of neuronal interactions and of acquisition parameters are investigated.

In chapter 2, GCM is applied to a study of switching visuomotor association tasks, to investigate the network of interacting regions underlying task switching. Inference on GCMs is extended to the random effects (RFX) level, to statistically assess the consistency of results over a group of 15 subjects.

In chapter 3 , high resolution diffusion tensor imaging of the human optic chiasm was performed ex vivo at ultra-high field. The optic chiasm, with its complex micro-structure where fibers kiss, cross, converge and curve is a challenge for the capabilities of the diffusion tensor model and tractography methods. Since the macroscopic connectivity through the optic chiasm is well known, it is ideal candidate for validation of DTI tractography approaches in resolving inter-regional connectivity.

In the general discussion, Granger causality mapping and diffusion tensor imaging are discussed in the context of effective connectivity studies. The concrete merits and problems of each technique are discussed. Recommendations for possible improvements and future extensions are made. Finally, an outlook is given on how functional connectivity methods and effective connectivity methods can be combined with anatomical information gained from diffusion weighted MR and tractography in studies of functional integration. 


\section{References}

Basser PJ, Mattiello J, LeBihan D (1994) Estimation of the effective self-diffusion tensor from the NMR spin echo. J Magn Reson B 103: 247-254.

Basser PJ, Pajevic S, Pierpaoli C, Duda J, Aldroubi A (2000) In vivo fiber tractography using DTMRI data. Magn Reson Med 44: 625-632.

Buchel C, Friston K (2000) Assessing interactions among neuronal systems using functional neuroimaging. Neural Netw 13: 871-882.

Buchel C, Friston KJ (1997) Modulation of connectivity in visual pathways by attention: cortical interactions evaluated with structural equation modelling and fMRI. Cereb Cortex 7: 768778.

Catani M, Howard RJ, Pajevic S, Jones DK (2002) Virtual in vivo interactive dissection of white matter fasciculi in the human brain. Neuroimage 17: 77-94.

Conturo TE, Lori NF, Cull TS, Akbudak E, Snyder AZ, Shimony JS, McKinstry RC, Burton H, Raichle ME (1999) Tracking neuronal fiber pathways in the living human brain. Proc Natl Acad Sci U S A 96: 10422-10427.

Friston K (1994) Functional and Effective Connectivity in Neuroimaging: A Synthesis. Hum Brain Mapp 2: 56-78.

Friston K (2002) Beyond phrenology: what can neuroimaging tell us about distributed circuitry? Annu Rev Neurosci 25: 221-250.

Friston KJ, Buchel C (2000) Attentional modulation of effective connectivity from V2 to V5/MT in humans. Proc Natl Acad Sci U S A 97: 7591-7596.

Friston KJ, Harrison L, Penny W (2003) Dynamic causal modelling. Neuroimage 19: 1273-1302.

Goebel R, Roebroeck A, Kim DS, Formisano E (2003) Investigating directed cortical interactions in time-resolved fMRI data using vector autoregressive modeling and Granger causality mapping. Magn Reson Imaging 21: 1251-1261.

Harrison L, Penny WD, Friston K (2003) Multivariate autoregressive modeling of fMRI time series. Neuroimage 19: 1477-1491.

McIntosh AR, Gonzalez-Lima F (1994) Structural Equation Modeling and Its Application to Network Analysis in Functional Brain Imaging. Human Brain Mapping 2: 2-22.

Mori S, Crain BJ, Chacko VP, van Zijl PC (1999) Three-dimensional tracking of axonal projections in the brain by magnetic resonance imaging. Ann Neurol 45: 265-269.

Poupon C, Clark CA, Frouin V, Regis J, Bloch I, Le Bihan D, Mangin J (2000) Regularization of diffusion-based direction maps for the tracking of brain white matter fascicles. Neuroimage 12: 184-195.

Roebroeck A, Formisano E, Goebel R (2005) Mapping directed influence over the brain using Granger causality and fMRI. Neuroimage 25: 230-242.

Wakana S, Jiang H, Nagae-Poetscher LM, van Zijl PC, Mori S (2004) Fiber tract-based atlas of human white matter anatomy. Radiology 230: 77-87. 


\title{
Chapter 1
}

\section{Mapping directed influence over the brain using Granger causality and fMRI}

\begin{abstract}
We propose Granger causality mapping (GCM) as an approach to explore directed influences between neuronal populations (effective connectivity) in fMRI data. The method does not rely on a priori specification of a model that contains pre-selected regions and connections between them. This distinguishes it from other fMRI effective connectivity approaches that aim at testing or contrasting specific hypotheses about neuronal interactions. Instead, GCM relies on the concept of Granger causality to define the existence and direction of influence from information in the data. Temporal precedence information is exploited to compute Granger causality maps that identify voxels that are sources or targets of directed influence for any selected region-ofinterest. We investigated the method by simulations and by application to fMRI data of a complex visuomotor task. The presented exploratory approach of mapping influences between a region of interest and the rest of the brain can form a useful complement to existing models of effective connectivity.
\end{abstract}

Based on: Roebroeck A, Formisano E, Goebel R (2005) Mapping directed influence over the brain using Granger causality and fMRI. Neuroimage 25: 230-242. 
Functional brain imaging has contributed substantial insights into the neural correlates of human information processing and cognitive operations. Most research with positron emission tomography (PET) and functional magnetic resonance imaging (fMRI) has focused on relevant information about where information is processed in the human brain. To improve our understanding of how the brain processes information, more knowledge about the interactions of activated brain areas, i.e. functional integration (Friston, 2002) is needed. The investigation of functional integration with PET and fMRI data has been pioneered by several researchers (Friston, Frith and Frackowiak, 1993; Friston, Frith, Liddle et al., 1993; Horwitz, 1990; Horwitz et al., 1992; McIntosh et al., 1993). Their work has led to a rapid increase in investigations of functional integration with PET and fMRI for various cognitive or sensorimotor tasks. A model-free approach to study functional integration is to investigate the correlation between measured time courses of different brain areas, which has been referred to as functional connectivity (Friston, Frith, Liddle et al., 1993). However, functional connectivity is ambiguous with respect to underlying directed interactions that generated the observed correlations. Effective connectivity, defined as the influence one neuronal system exerts over another (Friston, 1994; Friston, Frith and Frackowiak, 1993), attempts to resolve this ambiguity by defining explicit statistical models of directed neuronal interactions. Recently, significant methodological advances in effective connectivity modeling have been made, such as the application to neuroimaging data of covariance structural equation modeling (Buchel and Friston, 1997; McIntosh and Gonzalez-Lima, 1994), nonlinear system identification techniques (Friston and Buchel, 2000), and Bayesian estimation of deterministic state-space models (Friston et al., 2003). However, effective connectivity methods proposed so far all require preselection of the interacting regions and assumptions about the existence and direction of influence between any two regions. These pre-specified models are useful in specifying and testing specific hypothesis concerning interactions between brain systems. However, an implicit problem in this type of approach is that misspecification of the models (e.g. by omission of an area that mediates or initiates interactions) can lead to erroneous conclusions.

Here we present a framework to map effective connectivity over the brain using vector autoregressive (VAR) modeling of fMRI time series in the context of Granger causality (Granger, 1969, 1980). Assuming that $x[n]$ and $y[n]$ are the measured time courses of two brain regions (or voxels), Granger causality quantifies the usefulness of unique information in one of the time series in predicting values of the other. Specifically, if incorporating past values of $x$ improves the prediction of the current value of $y$, we say that $x$ Granger causes $y$. Thus, temporal precedence is used to identify the direction of causality from information in the data. In our framework, time-resolved fMRI measurements provide topographical as well as temporal information about the brain areas subserving a cognitive task. Temporal precedence information is exploited to compute Granger causality maps (GCMs) that identify voxels that are sources or targets of directed influence for any selected region-of-interest. Thus, the method is exploratory in nature and does not require the specification of a directed graph model. 
Granger Causality analysis has been previously applied to electrophysiological animal data, specifically Local Field Potentials (LFPs) to detect influences at the neuronal population level (Baccala and Sameshima, 2001; Bernasconi and Konig, 1999; Bernasconi et al., 2000; Brovelli et al., 2004; Freiwald et al., 1999). More recently Granger causality and related frequency domain measures have also been applied to human EEG data (Hesse et al., 2003; Kaminski et al., 2001). Furthermore, multivariate autoregressive models have recently also been applied to human fMRI data to model effective connectivity between preselected regions based on temporal order (Harrison et al., 2003), thus making implicit use of the concept of Granger causality. We have previously reported initial investigations of the application of Granger causality to fMRI data (Goebel et al., 2003, 2004). However, there are two potential obstacles for the successful application of Granger causality to fMRI signals. First, fMRI only provides indirect access to the neuronal responses and information on dynamic interactions between neuronal populations may be irremediably lost or distorted by hemodynamic blurring of the neuronal responses. Second, the applicability of Granger causality may also be limited because of the relatively low temporal resolution of data acquisition techniques conventionally employed. In order to investigate the constraints posed by hemodynamics and temporal sampling, we performed a series of simulation studies. Our simulations show that it is possible to recover directed neuronal influences from the fMRI signal if short volume repetition times (TRs) are used. After describing the simulation studies, we describe an application of Granger causality mapping to fMRI data for a dynamic visuomotor mapping task. To assess significance of the obtained GCMs, we developed thresholding methods based on bootstrapping techniques (Efron and Tibshirani, 1993) and the control of the false discovery rate for voxelwise tests (Genovese et al., 2002). The method identified directed interactions between inferotemporal, parietal, and premotor areas that underlie the performance of a complex visuomotor task.

\section{Theory}

The discrete zero-mean vector time-series $\mathrm{x}[n]=\left(\mathrm{x}_{1}[n], \ldots, \mathrm{x}_{\mathrm{M}}[n]\right)^{\top}$ can be modeled as a vector autoregressive (VAR) process of order $p$ (e.g. Kay, 1988):

$$
\mathbf{x}[n]=-\sum_{i=1}^{p} \mathbf{A}[i] \mathbf{x}[n-i]+\mathbf{u}[n]
$$

Where $u[n]$ is (multivariate) white noise. The matrices $A[]$ are called the autoregression (AR) coefficients because they regress $x[n]$ onto its own past. The VAR model can be thought of as a linear prediction model, that predicts the current value of $x[n]$ based on a linear combination of the most recent $p$ past values. Consequently, the current value of a component $\mathrm{x}_{\mathrm{i}}[n]$ is predicted based on a linear combination of its own past values and the past values of the other components. This shows the value of the VAR model in quantifying Granger causality between (groups of) components. 
Granger causality uses temporal precedence to identify the direction of causality from information in the data. Thus, given two time series $\mathrm{x}[n]$ and $\mathrm{y}[n]$, we can independently identify both influence from $x$ to $y$, and influence in the reverse direction with suitable models. Geweke (1982) has proposed a measure of linear dependence $F_{x, y}$ between $x[n]$ and $\mathrm{y}[n]$ which implements Granger causality in terms of vector autoregressive models (see Appendix A for details). $F_{x, y}$ is the sum of three components:

$$
\mathbf{F}_{\mathbf{x}, \mathbf{y}}=\mathbf{F}_{\mathbf{x} \rightarrow \mathbf{y}}+\mathbf{F}_{\mathbf{y} \rightarrow \mathbf{x}}+\mathbf{F}_{\mathbf{x} \cdot \mathbf{y}}
$$

The four measures take their values in the interval $[0, \infty)$, i.e. they are by construction nonnegative. $F_{x, y}$ is a measure of the total linear dependence between the series $x$ and $y$. If nothing of the value at a given instant of one can be explained by a linear model containing all the values (past, present, and future) of the other, $F_{x, y}$ will evaluate to zero. $F_{x \rightarrow y}$ is a measure of linear directed influence from $x$ to $y$. If past values of $x$ improve the prediction of the current value of $y$, then $F_{x \rightarrow y}>0$. A similar interpretation holds, of course, for $F_{y \rightarrow x}$. Thus, the two directed components, $F_{x \rightarrow y}$ and $F_{y \rightarrow x}$, use the arrow of time to decide on the direction of influence. However, the total linear dependence between $x$ and $y$ does not often consist fully of these directed components. Much of the total linear dependence can be contained in the undirected instantaneous influence $F_{x \cdot y}$ between them. Essentially, $F_{x \cdot y}$ quantifies the improvement in the prediction of the current value of $x$ (or $y$ ) by including the current value of $y($ or $x$ ) in a linear model already containing the past values of $x$ and $y$. From this symmetry it can be seen that $F_{x \cdot y}$ indeed contains no directional information at all. It represents residual correlations in the data that can not be assigned to causally directed influence based on the information in the data. In practice, nonzero values of $F_{x \cdot y}$ can be caused by directed influence between $x$ and $y$ at a finer time-scale than that at which $x$ and $y$ are observed (Granger, 1969, 1980). We investigated the usefulness and validity of these measures in investigating effective connectivity in fMRI data, both by simulations and by application to real fMRI data.

\section{Simulations}

Based on the observed high correlation between Local Field Potentials (LFPs) and the Blood Oxygenation Level Dependent (BOLD) response (Logothetis et al., 2001), we can approximate fMRI signals with a low-pass filtered and sub-sampled version of LFPs. Previous invasive electrophysiological studies have shown that statistical techniques based on VAR-modeling and Granger causality are capable of detecting directed interactions between neuronal populations as reflected in the dynamic structure of LFP signals (Bernasconi and Konig, 1999; Bernasconi et al., 2000; Brovelli et al., 2004; Freiwald et al., 1999). We used simulations to investigate whether and to what extent this capability is preserved with fMRI measurements. We assumed neuronal interactions to occur at the level of LFP signals and quantified the effects of 1) hemodynamics (i.e. filtering) and 2) fMRI image collection (i.e. temporal sampling of BOLD responses) on computed Granger causality measures (see Figure 1). The signals $\mathrm{x}[n]$ and $\mathrm{y}[n]$ of two 
interacting neuronal populations $\mathrm{X}$ and $\mathrm{Y}$ were generated as a realization of a bidimensional first-order VAR process with:

$$
\mathbf{A}[1]=\left[\begin{array}{cc}
-0.9 & 0 \\
I & -0.9
\end{array}\right], \Sigma=\left[\begin{array}{ll}
1 & 0 \\
0 & 1
\end{array}\right]
$$

This model has the benefit of being simple while still allowing achievement of the desired dynamic characteristics. The model was specified to have the following properties: First, it embodies an influence from $X$ (its first channel, the influencing brain region) to $Y$ (its second channel, the brain region being influenced) of a predetermined strength $I$ (ranging from 0.0 , no influence, to 0.5 , strong influence).

Second, by construction, there is no influence in the reverse direction from $Y$ to $X$, rendering the modeled interaction strictly unidirectional. Third, the influence from $X$ to $Y$ was manipulated to have an additional delay $D$ (ranging from 0 to $100 \mathrm{~ms}$.) representing the time that passes before the population dynamics in $X$ has an influence on $Y$ 's dynamics. Fourth, instantaneous dependence between X's signal $x[n]$ and Y's signal $y[n]$ was also absent by construction (i.e. the off-diagonal terms in $\Sigma$ are zero). Fifth, the autoregressive coefficients on the diagonal (i.e. connecting $\mathrm{x}[n]$ and $\mathrm{y}[n]$ to their own respective pasts) were set such that all of their spectral power is contained in the lower frequency ranges. Thus, the influence $X$ exerts on $Y$ was constructed to take place in the lower frequency ranges. This is in line with the expectation that high frequency dependencies between $\mathrm{x}[n]$ and $\mathrm{y}[n]$ are not detectable after passing through hemodynamics (essentially a low-pass filter) and being down-sampled in the data acquisition. This would in turn transfer to the interpretation that the directed influences detected in real fMRI data would depend on low-frequency signal fluctuations, perhaps caused by the experimental design. 

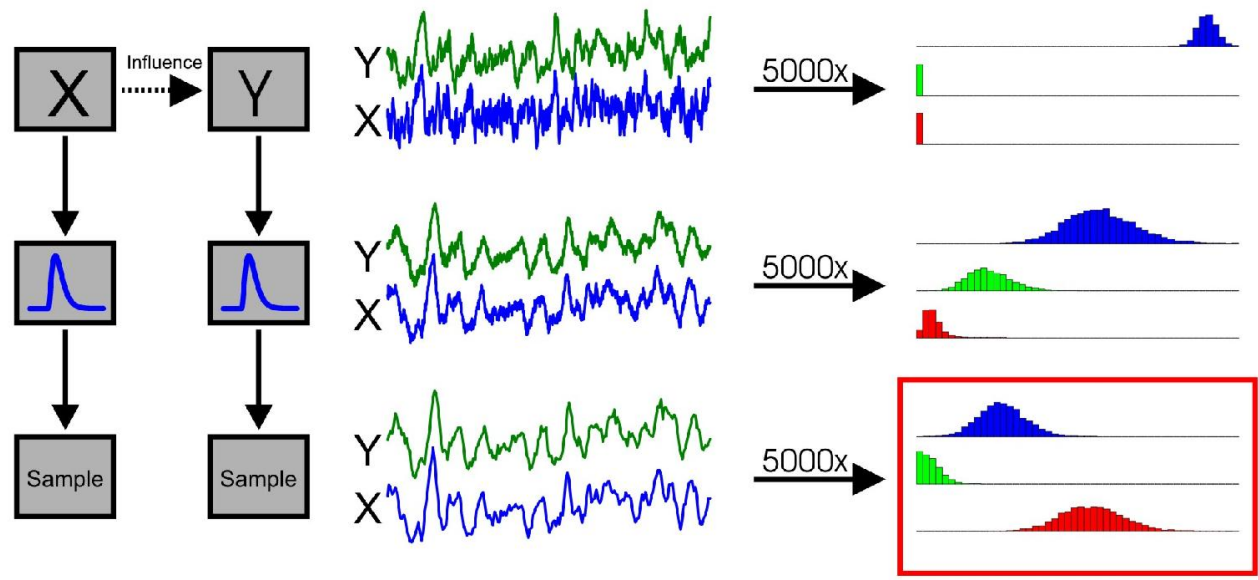

Figure 1: A schematic illustration of the procedure to generate simulated time series (in the leftmost column), examples of the generated series at various stages (in the middle column), and of resulting distributions of computed influence values for 5000 simulations (in the rightmost column). The top row depicts the generation of simulated local field potential (LFP) signals of $X$ and $Y$ at high temporal resolution. The simulation model implements a temporally directed influence from $X$ to $Y$. The middle row represents the filtering of the LFP signals through a canonical hemodynamic response model to obtain simulated blood oxygenation level dependent (BOLD) signals. The bottom row shows how a temporal down-sampling of the BOLD signals then gives the simulated fMRI signal. Influence measures $F_{x \rightarrow y}, F_{y \rightarrow x}$, and $F_{x \cdot y}$ can be computed from the generated time series at all three stages. If the simulation is repeated many times (e.g. 5000), distributions of the influence measures can be obtained. These are shown in the rightmost column, where the distributions of $F_{x \rightarrow y}$ values is shown in blue, $F_{y \rightarrow x}$ distributions are shown in green and $F_{x \cdot y}$ distributions are shown in red. The set of distributions for the simulated fMRI signal (in the red box) is of most interest in these investigations.

The time-step of the simulation was taken to be $10 \mathrm{~ms}$. In every simulation the model was simulated for 10000 time-steps (100 sec.), where additionally an initial $2000+D$ timesteps were simulated and later discarded to allow the system to enter a steady state, to introduce the delay $D$ and to avoid boundary effects in subsequent filtering. After simulation and introduction of additional delay the channels were individually filtered by convolution with a linear model of the Hemodynamic Response Function (HRF) based on a gamma function (Boynton et al., 1996). The tau parameter in this model, controlling the width of the HRF, was set to 0.5 , corresponding to short $(0.5 \mathrm{sec})$ stimulus durations (Liu and Gao, 2000), relevant for fast event-related designs. After individually normalizing the channels to zero mean and unit variance, $20 \%$ of white Gaussian noise was added, representing physiological noise in the BOLD response. Subsequently these simulated BOLD response signals were sampled every $S$ time-steps to simulate signal acquisition by the scanner with a whole volume TR of $S / 100$ seconds. After renormalizing the signals, another $20 \%$ of white Gaussian noise was added to represent measurement error and noise in the acquisition. Note, that noise enters the simulated 
system at three independent points. First, in the form of the innovation $\mathrm{u}[n]$ that drives the dynamics of the VAR model that generates the simulated LFPs. Second, noise is added at the level of the hemodynamics, mimicking imperfections in the transfer of neuronal signals to hemodynamic signals. Third, noise is added at the level of sampling by the MR-scanner, simulating additive instrumental noise. The resulting signals were used to compute influence measures $F_{x \rightarrow y}, F_{y \rightarrow x}$, and $F_{x \cdot y}$. The order $p$ of the estimated autoregressive models was set to that which minimized the Schwartz Criterion (SC), an order selection criterion, designed to trade-off the reduction in error-variance against the increase in the number of parameters (Luetkepohl, 1991).

Simulations were performed for systematic combinations of the levels of crucial parameters in the model: the strength of influence $(I=\{0.0,0.1,0.2,0.3,0.4,0.5\})$, the delay of influence $(D=\{0,1,2,3,4,5,6,7,8,9,10\})$, and the temporal sampling $(S=$ $\{10,20,30,40,50,60,70,80,90,100\})$. For each of the 660 possible combinations of these parameters a set of 5000 simulations was performed and influence measures $F_{x \rightarrow y}, F_{y \rightarrow x}$, and $F_{x \cdot y}$ were computed on the simulated sampled signals. Inference was performed in the context of the bootstrap (Efron and Tibshirani, 1993), which is based on empirically obtained null distributions (See Appendix B for details). Significance thresholds can be obtained both within the classical framework, controlling for Type I error (quantified by the proportion of false positives within all tests), and with methods controlling for the false discovery rate (FDR, the expected proportion of false positives within all tests with a positive result) and which are more appropriate in the context of mapping effects over an imaging volume (Genovese et al., 2002). An empirical null distribution for the simulations was formed by computing the influence measures on pairs of signals $\mathrm{x}[n]$ and $\mathrm{y}[n]$ from different simulations in the same set. Any dependence between two channels from different realizations of the model can only exist purely by chance and thus characterize the null hypothesis of no influence.

Figure $2 A$ shows the distributions of values obtained for $F_{x \rightarrow y}, F_{y \rightarrow x}$, and $F_{x \cdot y}$ in an exemplary set of simulations. Two observations can be made from these distributions. First the values found for $F_{x \rightarrow y}$ are, on average larger than those found for $F_{y \rightarrow x}$, reflecting the true influences present at the LFP level. Second, the values found for $F_{X: y}$ are markedly different from zero, pointing to instantaneous influence between $X$ and $Y$ not actually present at the LFP level. This finding is not surprising because we are applying Granger causality to time-series sampled at a courser interval than that at which interactions take place (Granger, 1969, 1980). The instantaneous influence term essentially quantifies partial correlation (functional connectivity) that cannot be assigned to influence in a certain direction purely from temporal information in the data. As a general pattern over all simulations, the levels of instantaneous influence found were large and increased with increasing sample interval. Since our interest is mainly in directed influences, the finding of instantaneous influences need not be a conceptual difficulty. When found in absence of additional directed influence, it merely points to the necessity to incorporate further assumptions (such as those in structural equation models (McIntosh and Gonzalez-Lima, 1994) or dynamic causal models (Friston et al., 2003)) when inference on effective connectivity is needed. 
A

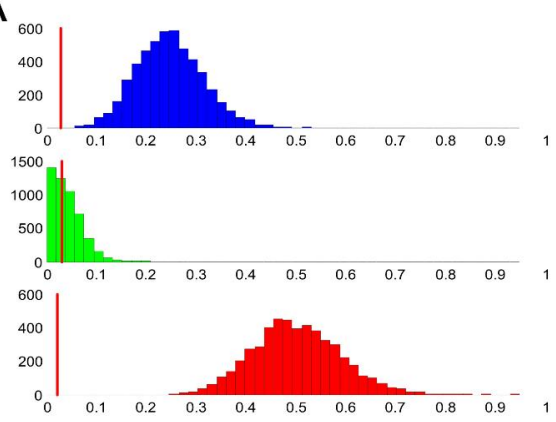

$\mathrm{C}_{2 \mathrm{sin}}$
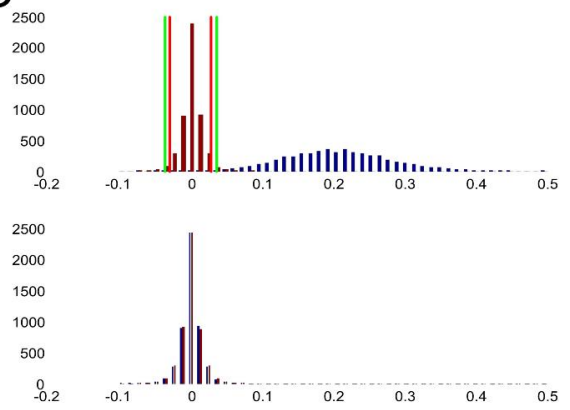

$\mathrm{B}_{\text {son }}$
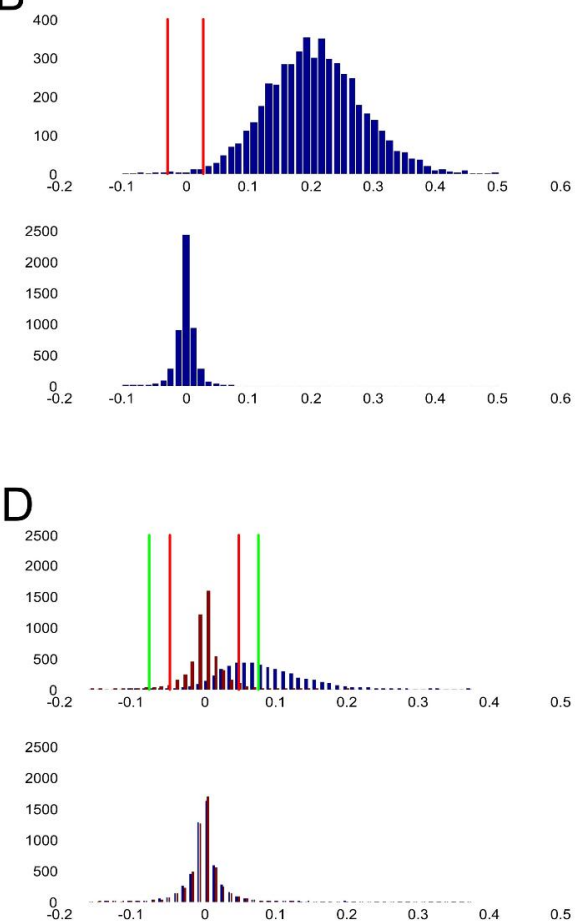

Figure 2: Distributions of influence measures resulting from 5000 simulations. In (A) histograms are shown for $F_{x \rightarrow y}$ values in blue in the upper panel, for $F_{y \rightarrow x}$ values in green in the middle panel, and for $F_{x \cdot y}$ values in red in the lower panel for 5000 simulations with $l=0.3, D=50 \mathrm{~ms}$, and $S=0.5 \mathrm{sec}$. The influence-difference terms for the same simulation set are shown in the upper panel of $(B)$, along with the associated empirical null distribution in the lower panel. The same distribution of difference terms is shown again in blue in the upper panel of (C) along with its null distribution in blue in the lower panel. Superimposed in red is the difference distribution of 5000 simulations with $l=0.0$ (and $D$ and $S$ as before) with its associated null distribution in red in the lower panel. In (D) the same distributions are shown with $S=100(1.0 \mathrm{sec})$. Horizontal red lines indicate classical significance thresholds at $\alpha=0.05$, and horizontal green lines indicate FDR-based thresholds at $q=0.05$, based on the empirical null distributions. Thresholds in $C$ and $D$ were computed from the joined set of values for $l=0.3$ and $l=0.0$.

Thus we turn to inference on $F_{x \rightarrow y}$ and $F_{y \rightarrow x}$. Classical significance thresholds for $\alpha=0.05$ are shown in Figure 2A. All $F_{x \rightarrow y}$ values are above threshold, suggesting that there is good sensitivity in detecting influence from $X$ to $Y$ even after interference of hemodynamics and temporal down-sampling. However, a much larger proportion of $F_{y \rightarrow x}$ values are above threshold than the nominal 0.05 , showing that, in the context of fMRI data, inferences on directed influences based on the simple terms introduced above may be biased. Conceptually, the problem of unidirectional influence turning into bi- 
directional interaction (Wei, 1990) is due to the unavoidable loss of dynamic stochastic information in both channels of the system arising from low-pass filtering and downsampling of the signals. In other words, some part of the variance in $x[n]$ that could previously explained by X's past, is now explained by Y's past, since the relevant information in X's past is lost. This leads to an inflation of the $F_{y \rightarrow x}$ measure. A possible solution to this inference problem, suggested by the observed distributions of $F_{x \rightarrow y}$ and $F_{y \rightarrow x}$ is to perform inference not on their individual values, but on their difference $\left(F_{x \rightarrow y}-\right.$ $\left.F_{y \rightarrow x}\right)$. Positive values of this influence difference term would point to influence from $X$ to $\mathrm{Y}$, whereas negative values would indicate influence in the reverse direction. The distribution of $\left(F_{x \rightarrow y}-F_{y \rightarrow x}\right)$ for the same set of simulations as before is shown in Figure $2 \mathrm{~B}$, together with its empirical null distribution. It can be seen from the significance thresholds for a two-sided test for non-zero values that inference on the difference term behaves very well. A very large proportion of values $(>0.99)$ is significantly positive, again indicating very good sensitivity. Moreover, only a very small proportion (much smaller than 0.05 ) of values is significantly negative, indicating good specificity. The observation of a smaller than the nominal proportion of false positives was in fact repeated over the full range of values of $I, D$, and $S$. It should be noted, that the regained robustness in inference on unidirectional influences comes at the cost of a lack of sensitivity to true bi-directional interactions (see Discussion).

Because the main goal of our approach is to map directed influences over the whole brain, sensitivity and specificity were investigated further within the framework of FDRbased hypothesis testing (see Appendix B). In this testing framework methods are employed that control the FDR (the expected proportion of false positives within all tests with a positive result) even over a large number of tests, thus presenting an approach to the multiple comparison problem (Genovese et al., 2002). To evaluate performance of FDR-based inference on the influence difference terms, FDR-based thresholds were computed for sets of 10,000 simulations of $x[t]$ and $y[t]$. In half of the cases there existed influence from $X$ to $Y$ (with given strength $I$ and delay $D$ ), whereas in the other half there was no influence between the simulated signals (i.e. $l=0.0$ ). This forms a more realistic inference situation where cases of true influence must be detected within a larger set (e.g. an imaging volume) of dynamically varying signals. To quantify performance of the inference on the influence-difference terms, the empirical power (as a measure of sensitivity) was computed, for a given threshold, as the proportion of true positives within all tests, i.e. the proportion of values that were above threshold for the set with non-zero I. The empirical fdr (as a measure of specificity) for FDR-based thresholds was obtained as the proportion of false positives within all tests with a positive result. Figure $2 \mathrm{C}$ (upper panel) again shows the distribution of influence difference values obtained for the set of 5,000 interacting signals with superimposed the distribution of values obtained for the 5,000 signals without influence. FDR-based thresholds were computed for the full set of 10,000 simulations based on its empirical null distribution (shown in the lower panel of 2C). Classical thresholds were also computed for comparison. The same distributions are shown in Figure 2D for simulations with a larger sampling interval. Both the probability of type I error, for classical thresholds, and the empirical fdr, for FDR-based thresholds, are well under control. Again, this degree of specificity was observed over the full range of simulations. The FDR-based inference methods seem to adapt well to the data, since the threshold automatically adjusts to trade-off control over FDR against 
sensitivity, lowering when signal-noise separation is good (when sampling faster as in Figure $2 \mathrm{C}$ ), and increasing to more critical levels when separation is low (at the lower sampling rate in 2D).

The estimated optimal orders for the simulations, as defined by the order selection criterion (see Appendix A), showed an interesting pattern. In general, the order for an autoregressive model needed to capture the dynamics of the simulated fMRI signal was not always equal to the order of the model used to generate the LFPs (which was of order 1). Rather, the estimated optimal order for simulated fMRI time-series was dependent on the sample rate $S$. The distributions of optimal orders estimated for series with fast sampling were mostly relatively high (e.g. peaking at about 5 , and ranging from 2 to 8 , for the set of simulations with $I=0.3, D=5$, and $S=10$, i.e. $T R=100 \mathrm{~ms}$ ). As the sampling interval increases, the observed optimal orders decreased (e.g. peaking at 2 for the simulations in Figure $2 \mathrm{C}$, where $\mathrm{TR}=500 \mathrm{~ms}$, and showing almost exclusively an optimal order of 1 for the simulations in Figure 2D, where $T R=1000 \mathrm{~ms}$ ). Thus, the order selection criterion tends to select more complex models to capture the dynamics that remain at high sampling rates, even more complex than the original models because the original dynamics are distorted in the hemodynamic filtering and temporal downsampling. The selected models at low sample rates are less complex, since a lot of the dynamics is lost in the hemodynamics and sampling. At the more realistic sample-rates for whole-brain $\mathrm{fMRI}$ that were simulated (towards $1 \mathrm{sec}$.), the optimal order was almost exclusively 1.

A summary of the simulations is given in Figure 3, which plots sensitivity (computed as empirical power, the proportion of true positives), as a function of a range of values for $I, D$, and $S$. Three main observations can be made. First, an increase in the strength of influence, at given levels of influence delay and sampling interval, leads to a steady increase in the influence difference measure and, consequently, in the power to detect that influence. Second, increasing the delay of influence, when keeping influence strength and sampling interval constant, also has the effect of increasing the influence measure. The conjoint effects of strength and delay of influence seem to be roughly additive. Third, an increase in power also results from decreasing the sampling interval for influence of given strength and delay, where most power can be gained in the current simulations by decreasing the sampling interval from $1 \mathrm{sec}$. to $0.5 \mathrm{sec}$. 


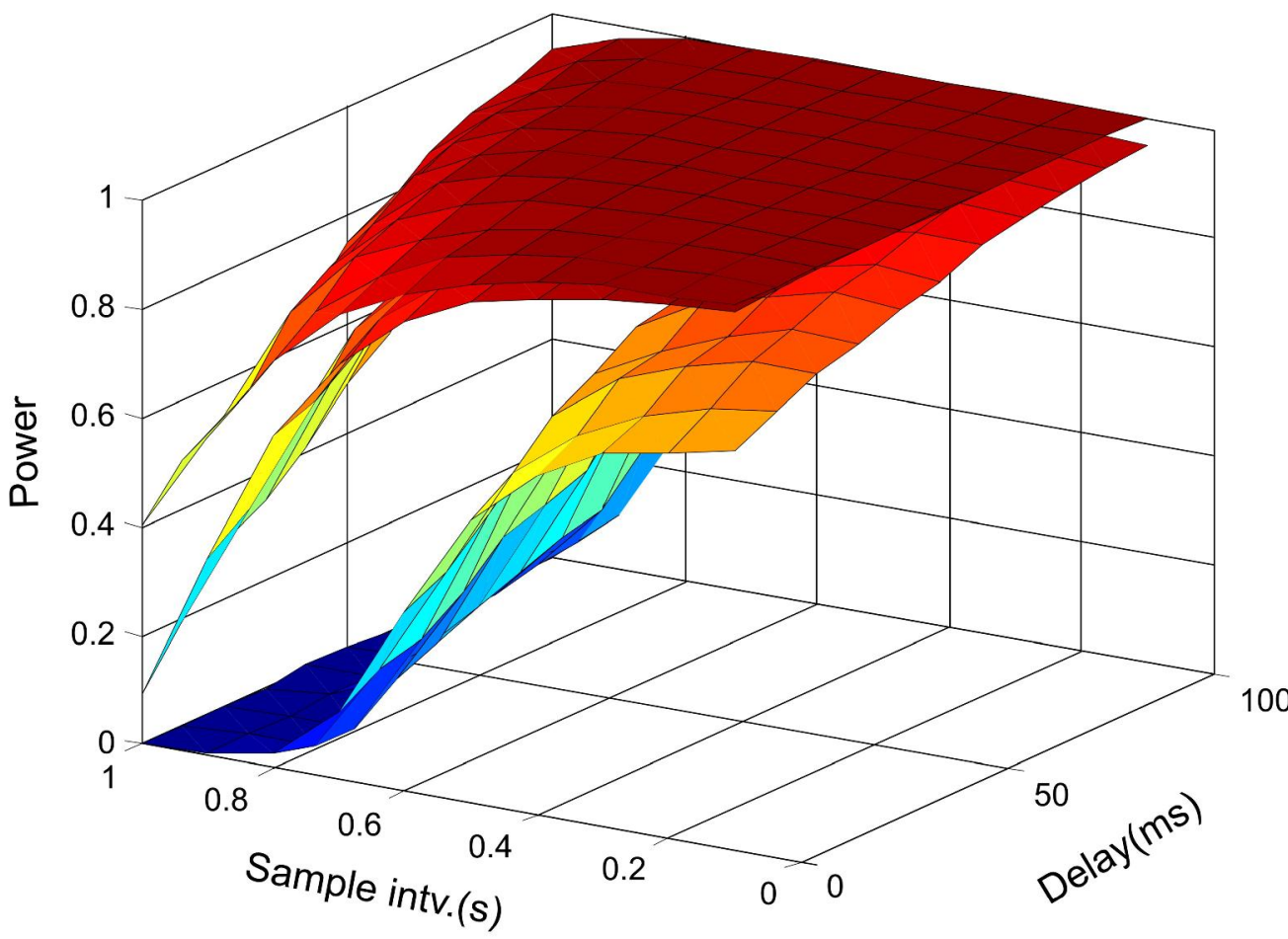

Figure 3: Graphical summary of simulation results. Three surface plots of Power (proportion of true influence cases that were above threshold), for $l=0.1$ (lower surface), $l=0.3$ (middle surface), and $I=0.5$ (upper surface), as a function of the sample interval $S$ (in seconds), and the influence delay $D$ (in milliseconds).

Overall, these simulations support a few important conclusions. First, naïve computation of Granger causality over fMRI signals as a measure of effective connectivity between neuronal populations can be misleading. The influence difference term, suggested here, proves to be a much more robust estimator of influence, on filtered and down-sampled signals, similar to the fMRI signal, at least in the case of unidirectional influence. Second, the proposed method is able to detect influence between neuronal populations in the $\mathrm{fMRI}$ signal even if the timescale and delay of the influence is smaller than the interval at which the data is sampled. However, the sensitivity to such interactions decreases rapidly with increasing sampling interval. Finally, the strength and the delay of influence have an additive effect on the computed influence difference term, making the interpretation of the absolute value somewhat ambiguous. A high computed influence difference term can arise through a strong influence, or one with a large delay, or both. 


\section{fMRI Data}

We applied GCM analysis to fMRI data obtained with a rapid event related design for a complex cognitive task. Two subjects performed a visuomotor mapping task in which two stimulus categories had to be mapped to two responses ("left" or "right"). The mapping of the two stimulus categories ("houses" and "faces") to the responses alternated periodically between the two possible mappings. A remapping cue indicated a change in the required stimulus-response mapping (S-R mapping) for the following trials. In addition to the face-stimuli and house-stimuli, pictures of objects appeared that required no response. This task was explicitly designed to engage sensory and motor related processes and executive control functions. The stimulus categories used are known to activate specific inferotemporal areas, the fusiform face area (FFA) for face stimuli (Kanwisher et al., 1997) and the parahippocampal place area (PPA) for house stimuli (Epstein and Kanwisher, 1998). Likewise, left and right hand button responses are known to be initiated in specific parts of right and left motor cortex. Importantly, correct performance on the task requires extensive interactions between different specialized systems in the brain at two distinct levels and temporal scales. First, within every single trial a relatively fast transition of information has to take place from sensory areas involved in the identification of a stimulus to motor areas controlling the response hand. Probably the link between sensory and motor areas is not direct and additional systems intervene in the relay of information. Furthermore, performance of the correct response requires contextual information about the currently valid S-R mapping. Second, a change in the S-R mapping at the remapping cue requires executive control processes that must operate to change and then maintain representations of the contextual information. The systems involved in these control processes must continually influence areas involved in the trial-to-trial responses to a given stimulus. Therefore, they are expected to operate on a timescale larger than that of a single trial. The GCM analysis was focused on the identification of the areas and interactions involved in these executive control processes, since their detection would probably require less extreme sample rates.

\section{Methods and Materials}

The two participating subjects were right handed, had normal or corrected-to-normal vision. Subjects gave informed written consent. Images were acquired using a 3 Tesla scanner ("Trio", Siemens, Erlangen, Germany). Functional images were acquired with a T2* weighted echo planar sequence (echo time (TE) $28 \mathrm{~ms}$, volume repetition time (TR) $1000 \mathrm{~ms}$, field of view $224 \mathrm{~mm}$ x $224 \mathrm{~mm}, 64$ × 64 matrix, giving $3.5 \mathrm{~mm} \times 3.5 \mathrm{~mm}$ inplane resolution). The images consisted of 18 oblique transverse slices (interleaved acquisition), $5 \mathrm{~mm}$ thick with a $1 \mathrm{~mm}$ inter slice gap. Both the fast-switching (FS) condition and slow-switching (SS) condition comprised a full acquisition run, each of which were performed twice by both subjects. For the SS runs 540 volumes were acquired; for the FS runs 500 volumes were scanned. Structural images were acquired using a T1 MPRAGE sequence (echo time $4 \mathrm{~ms}, 256 \times 256 \times 192$ matrix, 1 x 1 x $1 \mathrm{~mm}^{3}$ voxels). Stimulus presentation, response registration, and synchronization to the scanner acquisition were performed using the software program Presentation (Neurobehavioral 
systems, San Francisco, CA). In the FS condition, the S-R mapping changed 24 times (every 2 to 6 trials), while in the SS condition, the S-R mapping changed 8 times (every 15 trials). The mapping cue consisted of a $500 \mathrm{~ms}$ change in color of the fixation-cross (magenta for mapping 1, cyan for mapping 2). Trial-stimuli (5 face-pictures, 5 housepictures, 5 object-pictures) were shown for $120 \mathrm{~ms}$ with a stimulus onset asynchrony (SOA) of 2-6 seconds, synchronized to the volume acquisition of the scanner. The fastswitching runs contained 30 trials, each of faces, houses, and objects, balanced over the 5 different instances and pseudo-randomized with respect to the preceding SOA. The slow-switching runs contained 40 trials of each of the trial-stimuli. In both FS runs and SS runs, trial-stimuli were balanced over the two S-R mappings giving 30 required left and right hand responses in the FS runs and 40 required left and right hand responses on the SS runs. Feedback on the correctness of responses was given at every trial (500ms change to a green fixation cross for a correct response; red fixation cross for an incorrect response). Each of the two subjects performed two runs of each of the SS condition and the FS condition.

Imaging data were analyzed using BrainVoyager 2000 (Brain Innovation, Maastricht, The Netherlands). The anatomical volume was transformed to the Talairach coordinate system (Talairach and Tournoux, 1988). The cortical surface was reconstructed (Kriegeskorte and Goebel, 2001) and inflated for visualization of results. The time courses of activation of individual voxels were constructed from the functional images and corrected for the temporal difference in acquisition of different slices (slice scan time correction) using sinc interpolation. Subsequently, linear trends and low frequency components (up to and including 4 cycles in the time course) were removed prior to any analysis. Voxel time courses were then coregistered to the structural volume and transformed into Talairach space with a resolution of $3 \times 3 \times 3 \mathrm{~mm}$ using trilinear interpolation. No spatial or temporal smoothing was applied to the functional time courses.

Regional activations were analyzed using single subject General Linear Models (GLM) computed over multiple runs (fixed-effects analysis). Predictor functions for the mapping cue, the control stimulus, the face stimulus and the house stimulus, were constructed as box-car functions (value one at the single scan where the relevant event took place, value zero otherwise) filtered through a linear model of the BOLD response (Boynton et al., 1996). Regions of interest (ROI) were selected as activated regions in Fmaps for the contribution of the mapping cue predictor in the fast runs, or in the t-maps for the contrast of faces against houses computed over all runs (fast switching and slow switching).

Granger Causality Maps (GCM) were computed for a given reference (ROI) by computing the influence measures $F_{x \rightarrow y}, F_{y \rightarrow x}$, and $F_{x \cdot y}$, for every voxel from average time-course of the voxels in the ROI (as $x$ ) and the voxel time-course (as $y$ ). In accordance with the results from the simulations, the influence difference term $\left(F_{x \rightarrow y}-\right.$ $F_{y \rightarrow x}$ ) was then computed for every voxel to form the difference-GCM (dGCM), mapping influence to and from the ROI over the brain. The order of the autoregressive models used for computation of the influence measures was set to 1 , based on observed optimal orders in the simulations for a corresponding TR of $1 \mathrm{sec}$., and on exploratory analyses with the order selection criterion of this data and similar data-sets with the same TR. Before further inference and visualization of the difference GCMs they were masked with 
the thresholded (at 0.02) instantaneous GCM $\left(F_{x \cdot y}\right)$ as a simple first approach to remove some of the observed vessel effects. The reasoning is that vessels often have a large contribution to the directed GCMs whereas their contribution to the instantaneous GCMs is considerably more modest, probably because influence from regions to large draining vessels happens at larger time-lags. In contrast, cortical contributions to the directed GCMs always seem to be observed in conjunction with a large contribution to the instantaneous GCM. The GCMs were computed as pooled estimates separately over the two FS runs and the two SS runs for each subject. Thresholds on the map were computed using the bootstrap method and the false discovery rate, as explained above and in Appendix B. Empirical null distributions were obtained by recomputing the GCMs with a simple version of a 'block-randomized' reference time-course. The reference timecourse was split in two and the two halves were interchanged. Since influence from observations of the reference $\mathrm{ROI}$ in the first half of the run to other voxels in the last half of the run (or vice versa) can only be due to chance, the resulting distribution of values in the computed difference GCM characterizes the null hypothesis of $F_{x \rightarrow y}-F_{y \rightarrow x}=0$.

Event-related BOLD responses were estimated by a deconvolution technique that can be formulated as a General Linear Model. Delta-function predictors are formed for every peri-stimulus scan for all relevant stimuli. Provided that the experimental design is suitable (properly randomized stimulus order with an SOA randomized in multiples of the volume TR), and the assumption of linearity of the BOLD response is not heavily violated, the resulting regression coefficients characterize the event-related BOLD response.

\section{Results}

Figure 4A and $4 \mathrm{~B}$ show the dGCMs for a face-selective region in the left infero-temporal cortex, identified by location and selectivity as the fusiform face area (FFA), for subjects 1 and 2 respectively. Regions shown in green have significantly negative influence difference terms and are thus indicated to be sources of influence to the reference ROI. Regions shown in blue have significantly positive influence difference terms and are thus indicated to be targets of influence from the reference ROI. The dGCMs show qualitatively similar patterns of influence from and to the left FFA in the two subjects. A strong influence on the left FFA from early visual areas including the calcarine sulcus can be seen in both subjects. Lateral premotor areas and medial supplementary and presupplementary motor areas also show a strong influence on the left FFA. The left FFA itself exerts its influence mainly on other parts of the bilateral inferotemporal cortex and on regions in bilateral posterior parietal cortex (PPC).

Figure 4 (next page): Thresholded difference GCMs for a face-selective region in the left fusiform gyrus for subject 1 in (A) and subject 2 in (B). The FDR-based threshold was set to $q=0.05$. The reference region is shown in red. Green areas have a significant negative difference term and are sources of influence to the reference region. Blue areas have a significant positive difference term and are targets of influence from the reference region. Event-related BOLD responses are shown for the circled areas in the calcarine sulcus (in green), the fusiform gyrus (the reference area, in red) and the intra-parietal sulcus (in blue) for both the Cue stimulus and the Face stimulus. Vertical bars indicate estimated standard errors. 

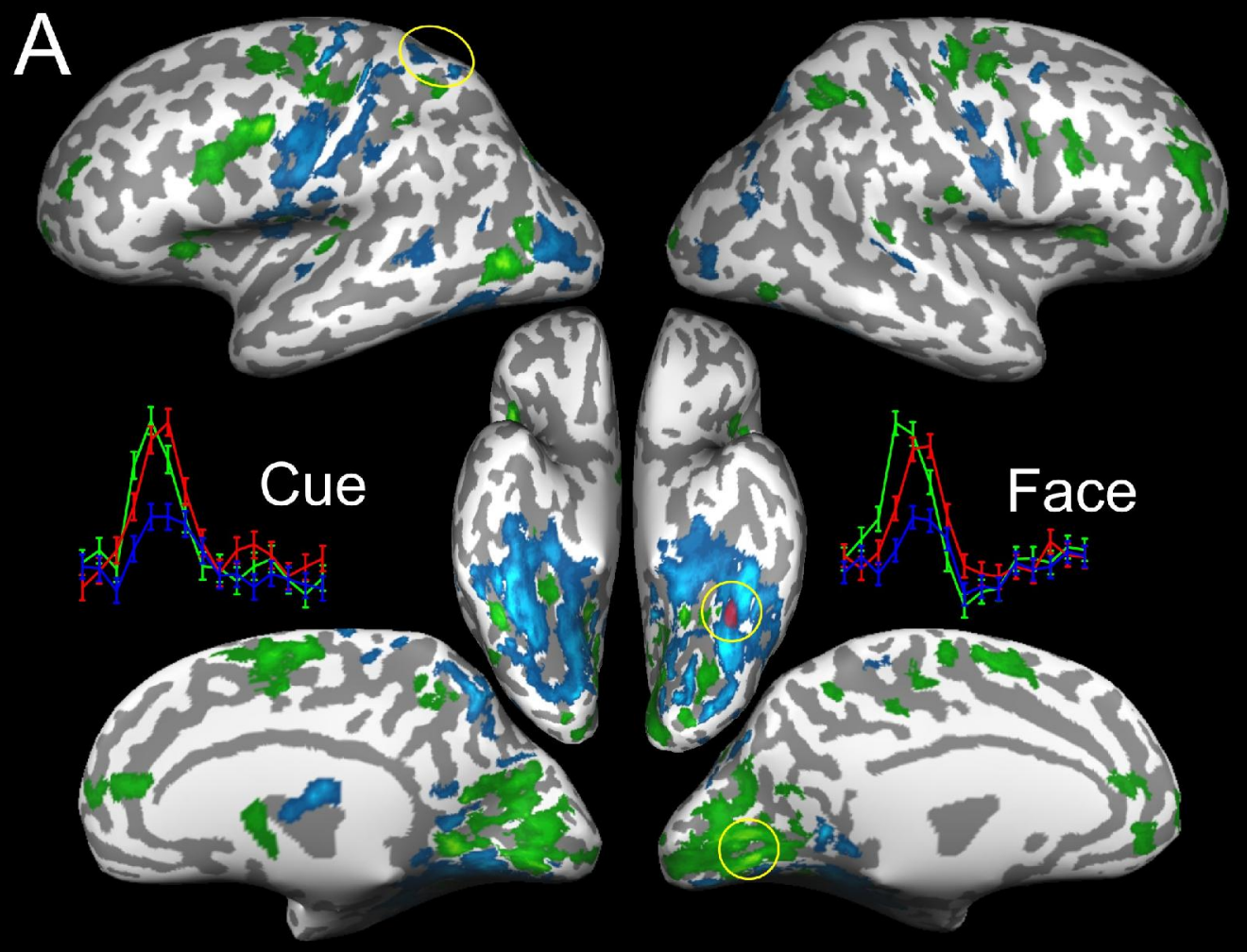

B

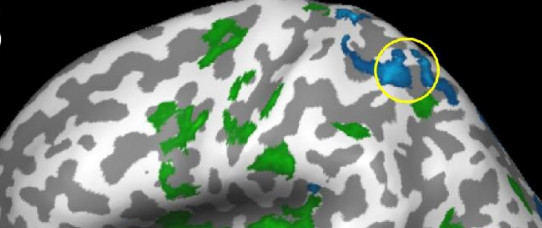

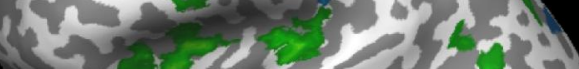

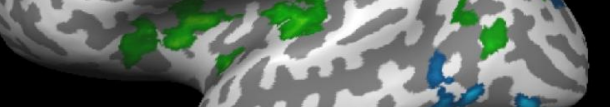
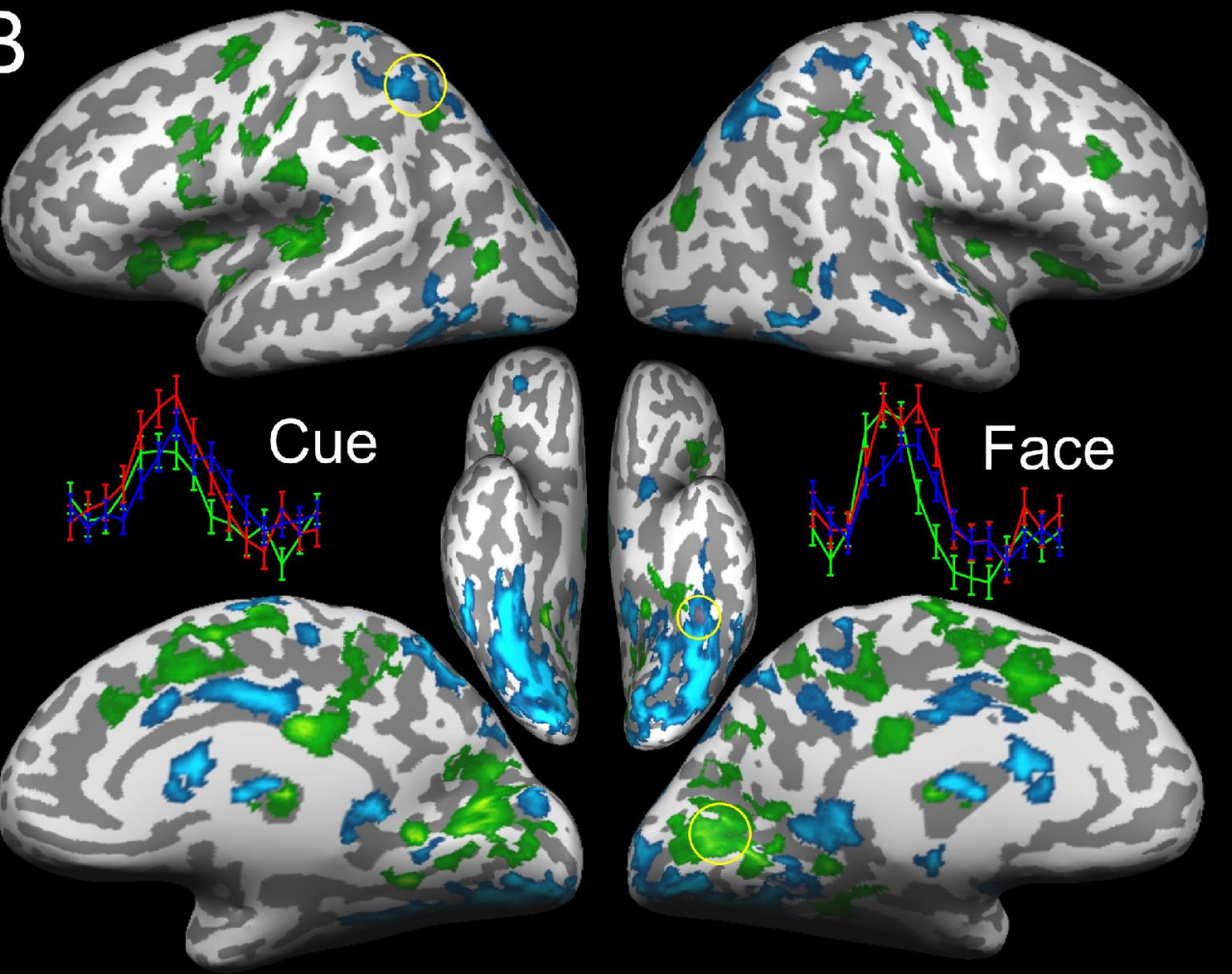
To aid in the interpretation of the maps a post-hoc deconvolution analysis was performed on the time-courses of selected foci in the maps. Event-related averages are shown for selected regions including the reference $\mathrm{ROI}$, synchronized to the occurrence of the remapping cue and the face stimulus. This post-hoc analysis provides valuable insight into the interpretation of the GCMs. It shows that the signal of influence sources in the maps rises and peaks before the signal of the reference region. The signal-rise and signal-peak of the reference region, in turn, precedes those of the influence targets. This observation indicates an agreement between fMRI mental chronometry (Formisano and Goebel, 2003; Menon et al., 1998) and GCM to the extent that the temporal precedence in stimulus-locked signal variation appears to be a contributing factor to the GCMs. It should be noted that GCMs also reflect the contributions from stochastic signal dependencies that are not strictly stimulus-locked, since they are computed over large time-segments within an experimental run.

An important issue that should be addressed when relying on temporal precedence between fMRI signals from different sites in the brain is the variability of the hemodynamics over the brain (Formisano and Goebel, 2003; Saad et al., 2001). More precisely, one should rule out the possibility that influence found from one area to another based on temporal difference in signal variation is due to a systematic difference in the hemodynamic lag at the two areas. A possible approach to exclude this confound is to show that the measured influence varies with experimental condition or cognitive context. The reasoning is that structural differences in hemodynamics persist over different conditions or contexts, so that any observed systematic variation with condition or context, should be due to changes in the neuronal population interactions. Thus, in the presence of such experimentally modulated influence, one can more reliably conclude that the measured influences reflect true neuronal interactions. To this end, we investigated differences between the dGCMs found in the fast switching (FS) condition and the slow switching (SS) condition. FS runs were of a similar length as the SS runs and contained a similar number of face, house, and control stimuli. However, the number of switches in the S-R mapping in the FS runs was three times that in the SS runs. Thus, although the stimuli, responses, and the general task were the same in both conditions, the FS runs created a much more engaging context, in which the subjects were required to exert a higher degree of executive control in order to switch the S-R mapping every few trials. Thus, it was hypothesized that this difference in task requirements and the ensuing cognitive context would be reflected in a difference in interactions between areas that coordinate the required executive control. Figure $5 \mathrm{~A}$ and $\mathrm{B}$ show, for the $\mathrm{FS}$ runs and the SS runs respectively, the dGCMs for subject 1 for a region in left posterior parietal cortex (PPC), thresholded at the same level. The reference region in left PPC was found to be highly activated at remapping cues, together with lateral premotor and prefrontal regions, medial supplementary motor regions and other parietal areas.

Figure 5 (next page): Thresholded difference GCMs for a region in the left intraparietal sulcus for subject 1 for the fast-switching runs in $(A)$ and the slow-switching runs in $(B)$ as in Figure 4. The FDR-based threshold was set to $q=0.05$ for both $A$ and $B$. Event-related BOLD responses are shown for the circled areas in the inferior part of the left precentral sulcus (in green), the left PPC (the reference area, in red) and the superior part of the left precentral sulcus (in blue) for both the Cue stimulus and the House stimulus. 
A

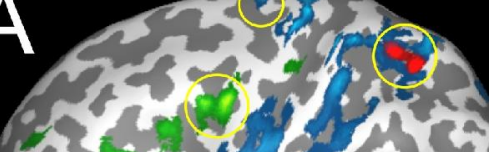

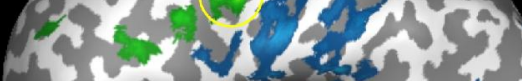

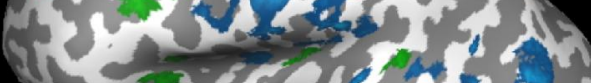
- 20 , 404 Whe cue the

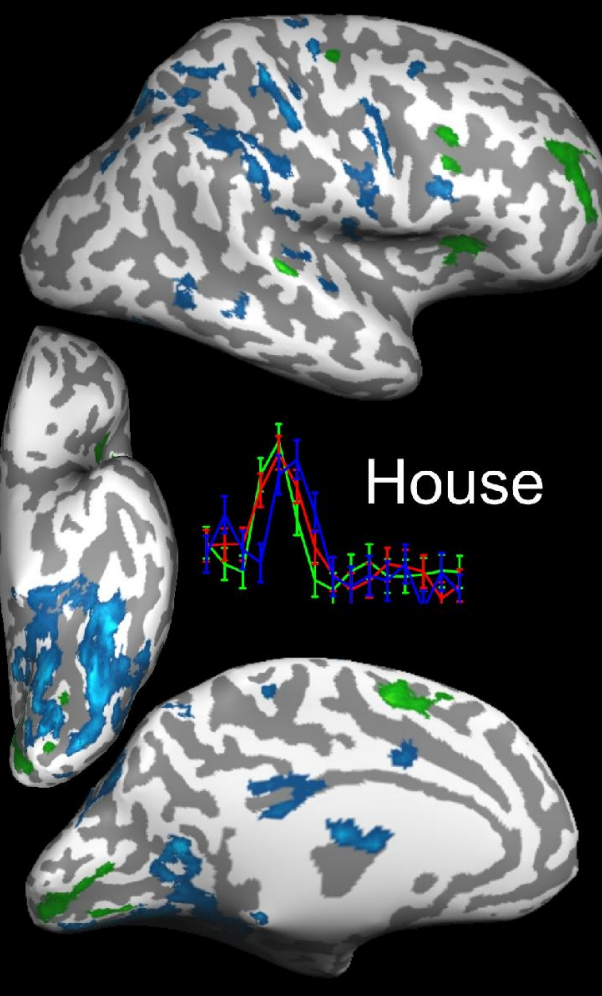

B

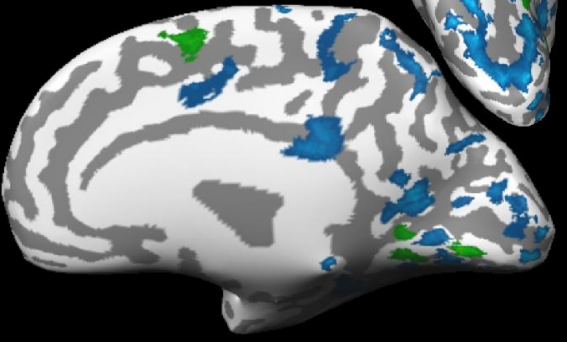

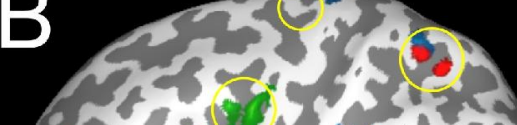

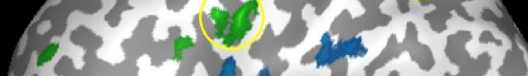
(1)
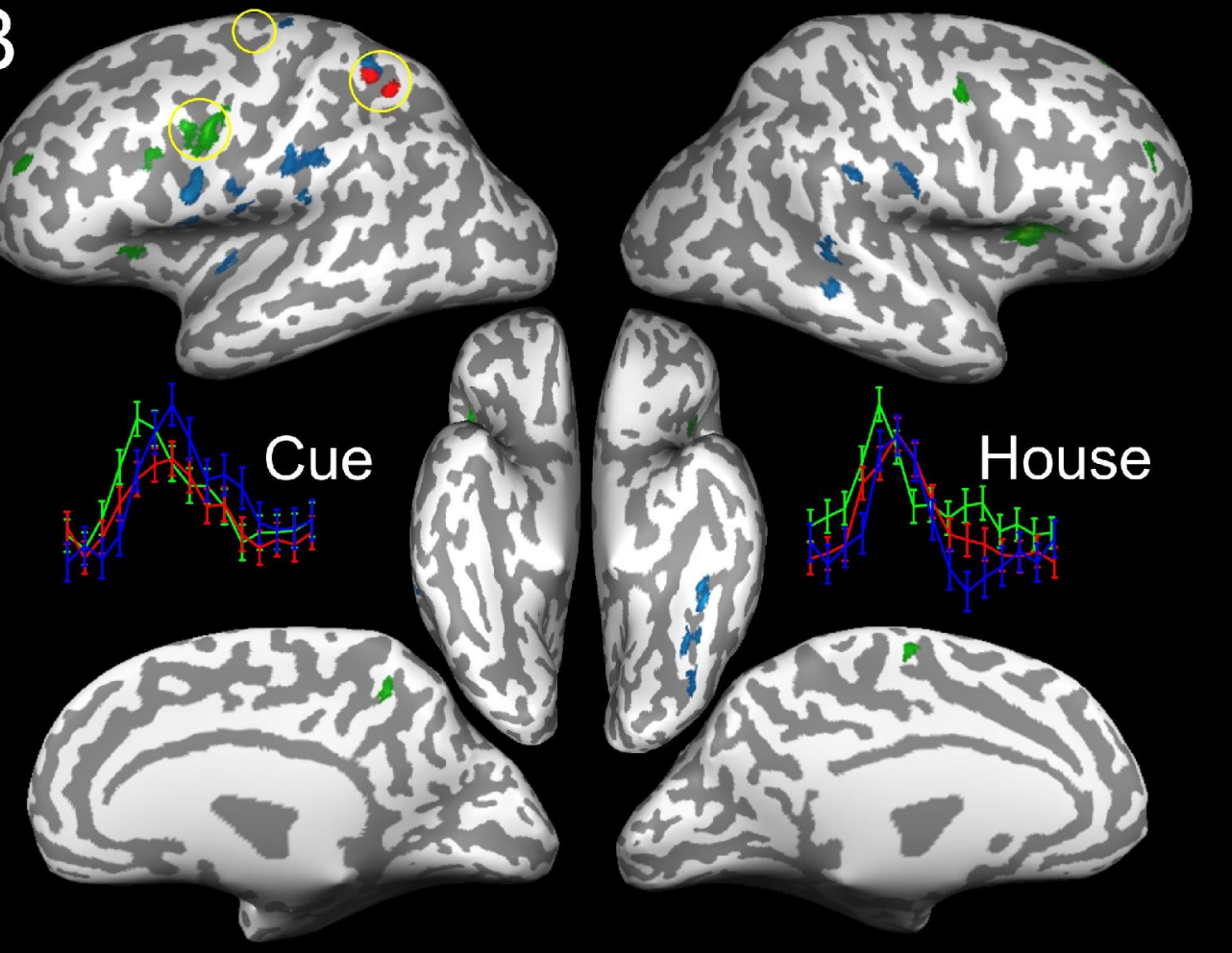
Overall, the dGCMs for FS runs and SS runs look qualitatively very similar but differ in intensity, suggesting that the degree of interaction of the left PPC with other sites in the brain is different. In the FS runs the left PPC is seen to be influenced mainly by premotor and prefrontal regions and presupplementary motor regions (left more strongly than right). There is also influence from bilateral insula regions and small clusters around the calcarine sulcus. The left PPC region itself exerts its influence mainly on large parts of the inferotemporal cortex, lateral motor and premotor areas and inferior and superior parietal areas. In the SS runs, the influence from some of the premotor, prefrontal, and insula regions remains, though mostly at a lower intensity. The influence from the reference region on some of inferior parietal and inferotemporal regions also remain in the SS runs at a lowered level. Overall, it can be seen that the change in cognitive context strongly modulates the intensity of the maps. Figure $5 \mathrm{~A}$ shows the event related responses for the remapping cue and the house stimulus in the FS runs. These responses reflect the temporal order relations implied by the GCM. In Figure 5B, the lower intensity of the maps in the SS runs is reflected in a less clearly structured relationship between the event related averages.

\section{Discussion}

We have proposed Granger causality mapping (GCM) as an approach to explore directed influences between neuronal populations in fMRI data. The method does not rely on a priori specification of a 'structural' or 'anatomical' model that contains preselected regions and connections between them. This distinguishes it from other effective connectivity approaches, such as covariance structural equation modeling (Mclntosh and Gonzalez-Lima, 1994) and dynamic causal modeling (Friston et al., 2003), that aim at testing or contrasting specific hypotheses about neuronal interactions. Instead, GCM relies on the concept of Granger causality to define the existence and direction of influence between two stochastic time-series purely on the basis of temporal precedence in their interdependency. Granger causality can be formalized and tested using vector autoregressive (VAR) models that capture the joint temporal dynamics of several time-series. Effective connectivity approaches that are based on instantaneous regression equations relating only concurrent values, such as psychophysiological interactions (Friston et al., 1997) and structural equation modeling, discard possibly important temporal information in the data. It has been shown (Lahaye et al., 2003) that even in the context of functional connectivity, incorporating lagged values increases the sensitivity to detect relationships between typical fMRI time-series. Many of the more recent effective connectivity approaches are based on stochastic or deterministic dynamic models, capable of capturing temporal structure. The Volterra series representation (Friston and Buchel, 2000) characterizes interactions in a nonlinear convolution model relating multiple inputs to a single output. Thus dynamic nonlinear influences on a single region can be characterized. In the multivariate context, the vector autoregressive models used by Harrison et al. (2003) can quantify directed influences between all regions included in the model. Although temporal information is used to give direction to the influences, the set of interacting regions must be chosen beforehand. The same holds for dynamic causal models (Friston et al., 2003) that use deterministic 
state-space models to represent neuronal dynamics and interactions augmented with forward models of the regional hemodynamic response. Pre-specified models are very useful, even necessary, when a specific hypothesis about neuronal interactions must be tested. Furthermore, most methods making use of a predefined anatomical model, capture the dynamics of all included regions simultaneously in a full multivariate model. This allows them to characterize and infer on indirect influences and other more complicated influence pattern than the canonical 'one-to-many' pattern inherent to the (bivariate) Granger Causality Mapping approach presented here. However, inference on a hypothesis concerning part of the specified network is very sensitive to misspecification of the model. Especially the omission of areas or structures that mediate influences or form an additional source of influence can lead to spurious interactions. Furthermore, in early stages of investigation specific hypotheses about the exact network underlying performance of a cognitive task might not be readily available. As an exploratory method, Granger causality mapping can form an important complement to these hypothesis driven methods in helping to formulate directed graph models of regions and their interactions.

The fMRI signal is influenced by the intervention of hemodynamics and a relatively low temporal resolution with respect to the interactions of neuronal populations. Simulations showed that these intervening operations of low-pass filtering and downsampling can introduce bias in inference on ordinary Granger causality based statistics. However, it was shown that robust detection of unidirectional influence from one neuronal population to another is possible in the fMRI signal using the proposed influence-difference term. Interestingly, with sufficiently high sample rates high sensitivity could be obtained even for influences with moderate strength and delay, suggesting that in practice considerable power could be gained with faster acquisition schemes. In resolving uni-directional influences based on temporal precedence in signal fluctuations, Granger causality mapping relates to the approach of fMRI mental chronometry (Formisano and Goebel, 2003; Menon et al., 1998). In fMRI mental chronometry the onset latency of BOLD responses is used to resolve a sequence of processing stages. Granger causality mapping forms an extension to this method, in principle using temporal precedence not only in the stimulus locked onset of the BOLD response but also in the ongoing signal fluctuations. Indeed we observed that the latency of trial based BOLD responses largely agreed with the directionality discovered by the GCMs. An interesting question arising from these observations is to what amount the influences found are driven more by strictly stimulus-locked deterministic signal fluctuations or by ongoing stochastic fluctuations, perhaps more indirectly induced by the experimental design. By its very nature, an autoregressive model does not distinguish between these two sources of signal fluctuations. The dynamics of the signal-fluctuations in an autoregressive model are driven by the random error-process $\mathrm{u}[n]$, which is therefore often called the innovation-process. The causes of the fluctuations in the innovationprocess itself are not explicitly modeled and, therefore, remain unclear after the fitting of an autoregressive model to given data. Thus, it is only by the post-hoc deconvolution analysis that we could ascertain that stimulus-locked signal changes, as characterized by an event-related average, seem to be an important source of temporally delayed signal fluctuations captured by the autoregressive models. More generally, autoregressive models estimated on the fMRI signal do not allow a 'blind deconvolution' 
that reconstructs an estimate of neuronal population dynamics (e.g. LFPs) from the observed fMRI signal. This would require more complex models with physiologically feasible state-variables and parameters, and an invertible observation model that characterizes local hemodynamics (Friston et al., 2003 ; Riera et al., 2004).

A question that remains is how influence difference terms can be interpreted in the context of more complicated bi-directional interactions, such as top-down feedback. Consider, for instance, a cortical area that sends bottom-up influence to a down-stream region and simultaneously receives top-down modulation from that same area. In this case, a dGCM likely shows only the dominant direction of influence, and only has the capacity to detect changes in the dominant direction of information flow between tasks or conditions. However, in a slightly different case where the source of bottom-up influence and the target of top-down modulation are different but anatomically very close, the exploratory mapping approach can prove to be very useful. With sufficient spatial resolution, a dGCM can identify and distinguish these functionally different parts of the network that might otherwise have been lumped together.

An important consideration in any method that relies on temporal precedence in fMRI data is to discount systematic differences in the lag of the BOLD response as a cause of the results. Of primary concern here is the possibility of a systematic difference in the lag of the hemodynamic response between different brain structures. Such a systematic difference could yield spurious influences. We examined the modulation of influence by experimental demands and cognitive context as a way to rule out hemodynamics as a cause of the results. The pattern of influences for a left posterior parietal region was indeed shown to be modulated by a change to an experimental context that required less cognitive effort. Perhaps explicit modeling of these modulations as psychophysiological interactions in a dynamic context (Friston and Buchel, 2000; Harrison et al., 2003) can form a useful extension to the current approach. A possible further improvement in the applicability of the method is its combination with approaches that try to identify and remove the effects of large draining vessels in fMRI data. Large vessels could often be observed in the GCMs, especially before projection onto the cortical surface. There is certainly an influence from the hemodynamic signal measured in a cortical area to that of the vessels that drain it. However, since in neuroimaging studies it is generally the interactions between neuronal populations that are of interest, strategies to remove vessel-related effects from the GCMs would be a useful addition. One should consider that in the current implementation of GCM one cannot be absolutely certain that a detected influence between two areas is a direct influence. This means that the influence shown between two cortical regions in a dGCM could run via a third region. However, in this case that third region would also be expected to show up in the same map. In addition, computation of an additional dGCM with this region as the reference could reveal its intervening role, being a target of influence from one of the areas, and a source of influence to the other. Similar considerations apply for other situations with additional influence from areas not taken into account, such as cases of common input. Computing conditional GCMs based on conditional influence measures (Geweke, 1984), which include the activity of a third area into the VAR models to partial out its influences, is a possible further approach towards handling these cases. 
In summary, we think the exploratory approach of mapping influences between a region of interest and the rest of the brain will form a very useful addition to existing models of effective connectivity. The absence of structural assumptions in the form of an anatomical model, makes it a useful tool in exploring possible alternative anatomical models underlying performance of cognitive and sensorimotor tasks. Because of its reliance only on assumptions incorporated in the concept of Granger causality, it can clarify which interactions are supported by temporal precedence information in the acquired data, and which other interactions, highlighted only by instantaneous correlations, require explicit directional modeling. Especially in early phases of investigation and data analysis, our method can help formulate explicit hypotheses about functional networks that can later be tested with more hypothesis-driven approaches. 
Appendix A: Computation of the influence measures

Geweke's dependence measure $F_{x, y}$ (Geweke, 1982) can be defined using the (zero-lag) autocorrelation matrices of the residuals of the following three VAR models involving the $K$-dimensional series $\mathrm{x}[n]$ and L-dimensional series $\mathrm{y}[n]$ :

$$
\begin{aligned}
\mathbf{x}[n]=-\sum_{i=1}^{p} \mathbf{A}_{\mathbf{x}}[i] \mathbf{x}[n-i]+\mathbf{u}[n] & \operatorname{var}(\mathbf{u}[n])=\mathbf{\Sigma}_{1} \\
\mathbf{y}[n]=-\sum_{i=1}^{p} \mathbf{A}_{\mathbf{y}}[i] \mathbf{y}[n-i]+\mathbf{v}[n] & \operatorname{var}(\mathbf{v}[n])=\mathbf{T}_{1}
\end{aligned}
$$

and with $\mathbf{q}[n]=\left[\begin{array}{l}\mathbf{x}[n] \\ \mathbf{y}[n]\end{array}\right]$ :

$$
\mathbf{q}[n]=-\sum_{i=1}^{p} \mathbf{A}_{\mathbf{q}}[i] \mathbf{q}[n-i]+\mathbf{w}[n] \quad \operatorname{var}(\mathbf{w}[n])=\mathbf{Y}=\left[\begin{array}{ll}
\boldsymbol{\Sigma}_{2} & \mathbf{C} \\
\mathbf{C}^{T} & \mathbf{T}_{2}
\end{array}\right]
$$

Where $\mathrm{q}[\mathrm{n}]$ is $O$-dimensional (with $O=K+L$ ), $\Sigma_{1}$ and $\Sigma_{2}$ are $K$ by $K, \mathrm{~T}_{1}$ and $\mathrm{T}_{2}$ are $L$ by $L$, and $Y$ is $O$ by $O$. Although both $x[n]$ and $y[n]$ can both be vector time series, they were both scalar time series in these investigations, i.e. $K=L=1$. The residual correlation matrices $\Sigma_{1}, \Sigma_{2}$, and $\mathrm{Y}$, quantify how well we are able (using linear AR models) to predict current values of $x$ and $y$ from their past values. The measures of total linear dependence between $x$ and $y$, linear influence from $x$ to $y$, linear influence from $y$ to $x$, and instantaneous influence between $x$ and $y$ are defined to be, respectively (Geweke, 1982):

$$
\begin{gathered}
\mathbf{F}_{\mathbf{x}, \mathbf{y}}=\ln \left(\left|\mathbf{\Sigma}_{1}\right| \cdot\left|\mathbf{T}_{1}\right| /|\mathbf{Y}|\right) \\
\mathbf{F}_{\mathbf{x} \rightarrow \mathbf{y}}=\ln \left(\left|\mathbf{T}_{1}\right| /\left|\mathbf{T}_{2}\right|\right) \\
\mathbf{F}_{\mathbf{y} \rightarrow \mathbf{x}}=\ln \left(\left|\mathbf{\Sigma}_{1}\right| /\left|\mathbf{\Sigma}_{2}\right|\right) \\
\mathbf{F}_{\mathbf{x} \cdot \mathbf{y}}=\ln \left(\left|\mathbf{\Sigma}_{2}\right| \cdot\left|\mathbf{T}_{2}\right| /|\mathbf{Y}|\right)
\end{gathered}
$$

From these definitions it can be seen that it holds that:

$$
\mathbf{F}_{\mathbf{x}, \mathbf{y}}=\mathbf{F}_{\mathbf{x} \rightarrow \mathbf{y}}+\mathbf{F}_{\mathbf{y} \rightarrow \mathbf{x}}+\mathbf{F}_{\mathbf{x} \cdot \mathbf{y}}
$$

Here we are assuming that the finite order AR-models are valid descriptions of the timeseries $\mathrm{x}[n], \mathrm{y}[n]$, and $\mathrm{q}[n]$, which also implies the assumption that $\mathrm{q}[n]$ is wide sense stationary (WSS) and thus that $\mathrm{x}[n]$ and $\mathrm{y}[n]$ are jointly WSS. Since it holds that $\left|\mathrm{T}_{2}\right| \leq$ $\left|T_{1}\right|, F_{x \rightarrow y}$ will always be nonnegative. As we can interpret the determinant of a correlation or covariance matrix as a measure of generalized variance, $\left|T_{1}\right|$ is the generalized 
variance of the mean squared error in predicting $y[n]$ by a linear projection on its own past values $\{y[n-1], y[n-2], \ldots\}$. Therefore, $F_{x \rightarrow y}$ quantifies the reduction in this generalized variance obtained by adding past values of $x$ to the projection set. A similar interpretation holds for $F_{y \rightarrow x} . F_{x \cdot y}$ essentially quantifies the deviation of the residual correlation matrix $Y$ of the joint VAR model from being block-diagonal, and thus the extent to which there is residual instantaneous correlation between $\mathrm{x}[n]$ and $\mathrm{y}[n]$.

VAR models were estimated from simulated or experimental data using a version of the multivariate fast orthogonal algorithm specialized for the estimation of VAR models with the possibility for non-linear terms and time-varying coefficients (Bagarinao and Sato, 2002). To specify the order $p$ of the autoregressive models to be estimated, the Schwarz Criterion (SC) was used, which is an order selection criterion, constructed in a Bayesian context that trades off reduction in error-variance against increased model complexity, i.e. number of parameters (Luetkepohl, 1991). The SC for a given VAR model fit is a function of model order $p$, model dimension $D$, the residual correlation matrix $\Sigma$, and the number of observations $\mathrm{N}$, and is given as (Luetkepohl, 1991):

$$
S C(p)=\ln (|\Sigma|)+\frac{\ln (N)}{N} p D^{2}
$$

Where ' $|\Sigma|$ ' denotes the determinant of $\Sigma$. Evaluating $\operatorname{SC}(p)$ for a large range of orders, the optimal order is selected as that for which $\mathrm{SC}(p)$ is minimal. 


\section{Appendix B: Statistical inference}

Parametric inference was developed for the influence measures $F_{x \rightarrow y}, F_{y \rightarrow x}$, and $F_{x \cdot y}$ (Geweke, 1982). However, such inference does not extend to more general conditional measures of influence (Geweke, 1984), and is not valid when dealing with sampled or aggregated time-series (Wei, 1990). Thus, inference on computed influence measures was performed within the framework of the bootstrap methodology (Efron and Tibshirani, 1993). A large number $M$ of surrogate time-series are generated that are sufficiently 'like' the original in their dynamic and statistical properties and that satisfy the null hypothesis of no influence. Computation of the influence measures over these surrogates gives a bootstrap empirical distribution of values that characterizes the null hypothesis. The empirical $p$-value or achieved significance level (ASL) for a given influence statistic obtained from real data can be taken as the proportion of values in the empirical null distribution more extreme than this value. Inference can then proceed either within the classical framework, controlling for probability of type I error a or, alternatively, with methods controlling for the False Discovery Rate (FDR). A classical test for an influence term being larger than zero is performed by setting the significance threshold at the value in the empirical null distribution that separates the $\alpha^{*} M$ largest values from the rest. A two-sided test for an influence difference term $F_{x \rightarrow y}-F_{y \rightarrow x}$ (see text) being non-zero corresponds to setting a lower threshold at the value in the empirical null distribution of difference terms that separates the $(\alpha / 2)^{*} M$ smallest values from the rest, and a upper threshold at the separation of the $(\alpha / 2)^{*} M$ largest values.

When performing a large number $V$ of simultaneous statistical tests (e.g. over a large number of voxels in a statistical parametric map), one can alternatively employ methods that control for the FDR, the expected proportion of false positives among all tests for which the null hypothesis is rejected (Genovese et al., 2002). This has the advantage of dealing with multiple comparison problem, while retaining considerable power in the detection of effects and adapting to the noise level in the data. The FDRbased thresholds corresponding to a two-sided test for the influence difference term being non-zero at an accepted FDR level $q$ are obtained from the set of empirical $p$ values of the obtained statistics over all voxels. Empirical $p$-values for the influence difference terms were pooled for positive and negative terms by taking absolute values of both the true statistic distribution and the null distribution. The empirical $p$-value for a given (absolute) difference term was then obtained as the proportion of larger values in the (absolute) empirical null distribution. Subsequently, the FDR-based threshold is obtained from the $p$-values as follows. In the ordered collection of $p$-values, let $r$ be the largest $\mathrm{i}$ for which $\mathrm{P}[\mathrm{i}] \leq(\mathrm{i} / V)^{*}(\mathrm{q} / \mathrm{c}(V))$, then the threshold is set at the value corresponding to the $p$-value $P[r]$. The value of the constant $c(V)$ is determined by assumptions on the joint distribution of $p$-values over all voxels. Here, it was set to $c(V)=1$, which applies when the $p$-values at different voxels are independent and when noise is Gaussian with nonnegative correlation across voxels. Alternatively, it can be set to:

$$
c(V)=\sum_{i=1}^{V} 1 / i
$$

Which applies for any distribution of $p$-values over voxels. 


\section{References}

Baccala, L. A., and Sameshima, K., 2001. Partial directed coherence: a new concept in neural structure determination. Biol Cybern 84, 463-474.

Bagarinao, E., and Sato, S., 2002. Algorithm for vector autoregressive model parameter estimation using an orthogonalization procedure. Ann Biomed Eng 30, 260-271.

Bernasconi, C., and Konig, P., 1999. On the directionality of cortical interactions studied by structural analysis of electrophysiological recordings. Biol Cybern 81, 199-210.

Bernasconi, C., von Stein, A., Chiang, C., and Koenig, P., 2000. Bi-directional interactions between visual areas in the awake behaving cat. NeuroReport 11, 689-692.

Boynton, G. M., Engel, S. A., Glover, G. H., and Heeger, D. J., 1996. Linear systems analysis of functional magnetic resonance imaging in human V1. J Neurosci 16, 4207-4221.

Brovelli, A., Ding, M., Ledberg, A., Chen, Y., Nakamura, R., and Bressler, S. L., 2004. Beta oscillations in a large-scale sensorimotor cortical network: Directional influences revealed by Granger causality. Proc Natl Acad Sci U S A 101, 9849-9854.

Buchel, C., and Friston, K. J., 1997. Modulation of connectivity in visual pathways by attention: cortical interactions evaluated with structural equation modelling and fMRI. Cereb Cortex 7 , 768-778.

Efron, B., and Tibshirani, R. J., 1993. An Introduction to the Bootstrap. Chapman \& Hall, New York.

Epstein, R., and Kanwisher, N., 1998. A cortical representation of the local visual environment. Nature 392, 598-601.

Formisano, E., and Goebel, R., 2003. Tracking cognitive processes with functional MRI mental chronometry. Curr Opin Neurobiol 13, 174-181.

Freiwald, W. A., Valdes, P., Bosch, J., Biscay, R., Jimenez, J. C., Rodriguez, L. M., Rodriguez, V., Kreiter, A. K., and Singer, W., 1999. Testing non-linearity and directedness of interactions between neural groups in the macaque inferotemporal cortex. J Neurosci Methods 94, 105119.

Friston, K., 1994. Functional and Effective Connectivity in Neuroimaging: A Synthesis. Hum Brain Mapp 2, 56-78.

Friston, K., 2002. Beyond phrenology: what can neuroimaging tell us about distributed circuitry? Annu Rev Neurosci 25, 221-250.

Friston, K. J., and Buchel, C., 2000. Attentional modulation of effective connectivity from V2 to V5/MT in humans. Proc Natl Acad Sci U S A 97, 7591-7596.

Friston, K. J., Buechel, C., Fink, G. R., Morris, J., Rolls, E., and Dolan, R. J., 1997. Psychophysiological and modulatory interactions in neuroimaging. Neuroimage 6, 218-229.

Friston, K. J., Frith, C. D., and Frackowiak, R. S. J., 1993a. Time-Dependent Changes in Effective Connectivity Measured with PET. Hum Brain Mapp 1, 69-79.

Friston, K. J., Frith, C. D., Liddle, P. F., and Frackowiak, R. S. J., 1993b. Functional connectivity: The principle component analysis of large (PET) data sets. J Cereb Blood Flow Metab 13, 514.

Friston, K. J., Harrison, L., and Penny, W., 2003. Dynamic causal modelling. Neuroimage 19, $1273-$ 1302.

Genovese, C. R., Lazar, N. A., and Nichols, T., 2002. Thresholding of statistical maps in functional neuroimaging using the false discovery rate. Neuroimage $15,870-878$. 
Geweke, J. F., 1982. Measurement of Linear Dependence and Feedback Between Multiple Time Series. Journal of the American Statistical Association 77, 304-324.

Geweke, J. F., 1984. Measures of Conditional Linear Dependence and Feedback Between Time Series. Journal of the American Statistical Association 79, 907-915.

Goebel, R., Roebroeck, A., Kim, D. S., and Formansfer function and statistical assessment of significance. Biol Cybern 85, 145-157.

Kanwisher, N., McDermott, J., and Chun, M. M., 1997. The fusiform face area: a module in human extrastriate cortex specialized for face perception. J Neurosci 17, 4302-4311.

Kay, S. M., 1988. Modern spectral estimation: theory and application. Prentice Hall, Englewood Cliffs, NJ.

Kriegeskorte, N., and Goebel, R., 2001. An efficient algorithm for topologically correct segmentation of the cortical sheet in anatomical mr volumes. Neuroimage 14, 329-346.

Lahaye, P. J., Poline, J. B., Flandin, G., Dodel, S., and Garnero, L., 2003. Functional connectivity: studying nonlinear, delayed interactions between BOLD signals. Neuroimage 20, 962-974.

Liu, H., and Gao, J., 2000. An investigation of the impulse functions for the nonlinear BOLD response in functional MRI. Magn Reson Imaging 18, 931-938.

Logothetis, N. K., Pauls, J., Augath, M., Trinath, T., and Oeltermann, A., 2001. Neurophysiological investigation of the basis of the fMRI signal. Nature 412, 150-157.

Luetkepohl, H., 1991. Introduction to Multiple Time Series Analysis. Springer-Verlag, Heidelberg.

McIntosh, A. R., and Gonzalez-Lima, F., 1994. Structural Equation Modeling and Its Application to Network Analysis in Functional Brain Imaging. Human Brain Mapping 2, 2-22.

McIntosh, A. R., Grady, C. L., Ungerleider, L. G., Haxby, J. V., Rapoport, S. I., and Horwitz, B., 1993. Network analysis of cortical visual pathways mapped with PET. J Neurosci 14, 655-666.

Menon, R. S., Luknowsky, D. C., and Gati, J. S., 1998. Mental chronometry using latency-resolved functional MRI. Proc Natl Acad Sci U S A 95, 10902-10907.

Riera, J. J., Watanabe, J., Kazuki, I., Naoki, M., Aubert, E., Ozaki, T., Kawashima, R., 2004. A statespace model of the hemodynamic approach: nonlinear filtering of BOLD signals. Neuroimage $21,547-67$.

Saad, Z. S., Ropella, K. M., Cox, R. W., and DeYoe, E. A., 2001. Analysis and use of FMRI response delays. Hum Brain Mapp 13, 74-93.

Talairach, J., and Tournoux, P., 1988. isano, E., 2003. Investigating directed cortical interactions in time-resolved fMRI data using vector autoregressive modeling and Granger causality mapping. Magn Reson Imaging 21, 1251-1261.

Goebel, R., Roebroeck, A., Kim, D. S., and Formisano, E., 2003. Directed cortical interactions during dynamic sensory-motor mapping. In Duncan, J. (Eds.), Attention \& Performance XX. Oxford University Press, New York, pp. 439-462.

Granger, C. W. J., 1969. Investigating causal relations by econometric models and cross-spectral methods. Econometrica 37, 424-438.

Granger, C. W. J., 1980. Testing for causality: A Personal Viewpoint. Journal of Economic Dynamics and Control 2, 329-352.

Harrison, L., Penny, W. D., and Friston, K., 2003. Multivariate autoregressive modeling of fMRI time series. Neuroimage 19, 1477-1491.

Hesse, W., Moller, E., Arnold, M., and Schack, B., 2003. The use of time-variant EEG Granger causality for inspecting directed interdependencies of neural assemblies. J Neurosci Methods $124,27-44$. 
Horwitz, B., 1990. Simulating functional interactions in the brain: A model for examining correlations between regional cerebral metabolic rates. Int J Biomed Comput 26, 149-170.

Horwitz, B., Grady, C. L., V., H. J., Ungerleider, L. G., Schapiro, M. B., Mishkin, M., and Rapoport, S. I., 1992. Functional associations among human posterior extrastriate brain regions during object and spatial vision. Journal of Cognitive Neuroscience 4, 311-322.

Kaminski, M., Ding, M., Truccolo, W. A., and Bressler, S. L., 2001. Evaluating causal relations in neural systems: granger causality, directed trCo-planar stereotaxic atlas of the human brain: 3dimensional proportional system, an approach to cerebral imaging. Thieme, Stuttgart.

Wei, W. W. S., 1990. Time Series Analysis: Univariate and Multivariate Methods. Addison-Wesley, Redwood City. 


\title{
The interactions of parietal and premotor cortex during task switching
}

\begin{abstract}
In a fast event-related fMRI study four switching visuomotor association tasks were compared to investigate the regions underlying the different component processes: response selection, competition and inhibition and stimulus-response (S-R) association. Random effects Granger causality mapping (RFX GCM) was used to identify the consistent interactions between regions involved at the group level, and the modulation of these interactions by task-type and switching-context. The activation of the dorsal premotor cortex (PMd) was found to be specific to processes of response selection and inhibition. RFX GCM analysis showed a strong input to the PMd from the (pre-) supplementary motor areas (SMA/preSMA), implying a close connection between medial (pre)SMA and lateral PMd in the selection of responses, specifically in situation where conflict between competing responses has to be resolved. Input to the PMd from the striatum was seen when specific $S-R$ associations are made in a non-conflicting context. The superior parietal lobule (SPL) was activated when multiple (possibly conflicting) S-R associations have to be kept online, showing it to be involved in controlling sets of S-R associations. RFX GCM analysis showed that an important part of that role could be the top-down attentional modulation of visual processing in inferotemporal cortex to bias processing for the relevant stimulus categories given the currently active S-R association. Input to the SPL is primarily exerted from left vLPFC and from the inferior parietal lobule (IPL). Influence from the vLPFC seems to underlie high-level control where superordinate level representations of tasks-sets in VLPFC influence the lowerlevel processing and representation of S-R sets in SPL. Influence from the IPL could reflect processing to change and reconfigure $S-R$ associations at switches. This gives important insight into the complex brain dynamics underlying arbitrary visuomotor association tasks and task switching in general. Furthermore, RFX GCM was shown to be a useful tool in exploring interactions in unknown large-scale brain networks that underlie the performance of specific task.
\end{abstract}

Based on: Roebroeck A, Formisano E, Goebel R. The interactions of parietal and premotor cortex during task switching. In preparation. 
The ability to flexibly change behavior according to contextual cues is an important human cognitive capacity. This capacity has been investigated in cognitive psychology and neuroimaging investigations with the 'task switching' paradigm: a decision or response task is performed by the subject according to certain rules which change regularly. A cued change in task rules necessitates cognitive set shifting: changing the parameters of processing for decision making, or stimulus-response association. Cognitive set shifting, in conjunction with processes for error detection and correction and conflict monitoring, are commonly termed 'executive functions'. Well known behavioral phenomena highlight the additional processing needed to perform a given task in a switching paradigm (Monsell, 2003). Switch costs refer to the longer response times (RTs) and higher error rates (ERs) on a 'switch trial', after the task has changed, than on a 'repeat trial', where the task has stayed the same. Switch cost is generally reduced with advance knowledge of the task switch and more time to prepare for the switch trial. However, switch costs are usually not completely eliminated by arbitrarily long preparation times. The longer RTs and higher ERs that remain even at cued task changes with long preparation times are called 'residual switch costs'. Besides the transient costs at switch trials, there is also a long term effect on performance. Mixing cost refers to the longer RTs for a given task in a switching context (i.e. when it is 'mixed' with another task) than when that task is performed in isolation.

A commonly used task in clinical settings to assess executive functions and cognitive strategic flexibility is the Wisconsin Card Sorting Task (WCST), in which cards have to be matched according to one of a few possible comparison dimensions (e.g. the number of elements on a card, the color of the elements, or the shape of the elements). The comparison dimension to use is regularly changed without warning, at which point the subject must find and use the new dimension on the basis of trial-by-trial feedback. Neuroimaging investigations employing he WCST to investigate task switching have found a switch in comparison dimension to be associated with prominent activity in frontal and parietal areas (Asari et al., 2005; Konishi et al., 2002; Konishi et al., 1998; Monchi et al., 2001). Although a very useful and common task the WCST is also a very complex task that involves many components other than cognitive set shifting, such as feedback processing, strategic search, spatial attention, and memory. Modifications to the basic WCST have been made to try and separate these processes and their underlying brain activity, for instance switch cues and elimination of spatial components (Asari et al., 2005; Konishi et al., 2002).

Another attempt at having a more controlled task switching paradigm in neuroimaging studies has been the use of cued arbitrary visuomotor association tasks (Bunge, 2004; Cools et al., 2004; Crone et al., 2006; Koechlin et al., 2003; Shafritz et al., 2005; Wylie et al., 2006). In an arbitrary visuomotor association task (Wise and Murray, 2000), discrete visual stimuli without a prelearned association towards a preferred action are associated with simple discrete responses. In the context of a task switching paradigm, a set of such stimulus-response (S-R) associations is sometimes called a rule, and a switch in cognitive set is needed at a cued rule-change (Bunge, 2004; Crone et al., 2006). As in other task switching paradigms, switch trials in cued S-R association tasks 
prominently activate frontal cortical areas (premotor areas, dorso-lateral prefrontal areas, and ventrolateral prefrontal areas) and posterior parietal areas, together with various other cortical and subcortical systems, such as anterior cingulate cotex (Shafritz et al., 2005), the striatum (Cools et al., 2004; Crone et al., 2006; Shafritz et al., 2005), the anterior insula, and (pre)Supplementary Motor Area (preSMA) (Dove et al., 2000). Despite their clear design and relatively simple nature, changing visuomotor association tasks still involve a host of higher order cognitive processes besides that of cognitive set shifting, among which are: response selection, response planning, response inhibition, conflict detection and resolution, attentional selection, and memory. A few studies have aimed at separating and localizing these component processes in the brain and deepened our understanding of the role played in task switching by many of the neural structures involved.

However, the way in which each of these structures interact with each other and collectively work together to implement the behavioral capacity of task switching remains poorly understood. In other words, although the functional specialization of systems involved in task switching has been the target of much research in recent years, the functional integration of these systems has not been the target of neuroimaging investigations to date.

We performed an event-related fMRI study of task switching in arbitrary visuomotor switching tasks and used Granger Causality Mapping to investigate the interactions of involved brain-areas. Granger Causality Mapping (GCM) is an exploratory approach to the investigation of effective connectivity in fMRI data (Goebel et al., 2003; Roebroeck et al., 2005). Activity in a brain region $X$ Granger causes activity in a region $Y$ if the past activity (i.e. BOLD-signal) of $X$ can help in predicting the current activity of $Y$. fMRI Granger Causality Maps (GCMs) are statistical maps of the influence from a designated reference region to all other regions in the brain and from all other brain-regions the reference region. Thus, for a selected region of interest both outgoing influence to other targets in the brain, and incoming influence from other source-regions are mapped. Here, the approach was extended to perform random effects (RFX) inference on the group-level, rather than on individual subject data. This enables the investigation of consistent generalizable interactions between brain regions over subjects and their modulation by experimental factors. 


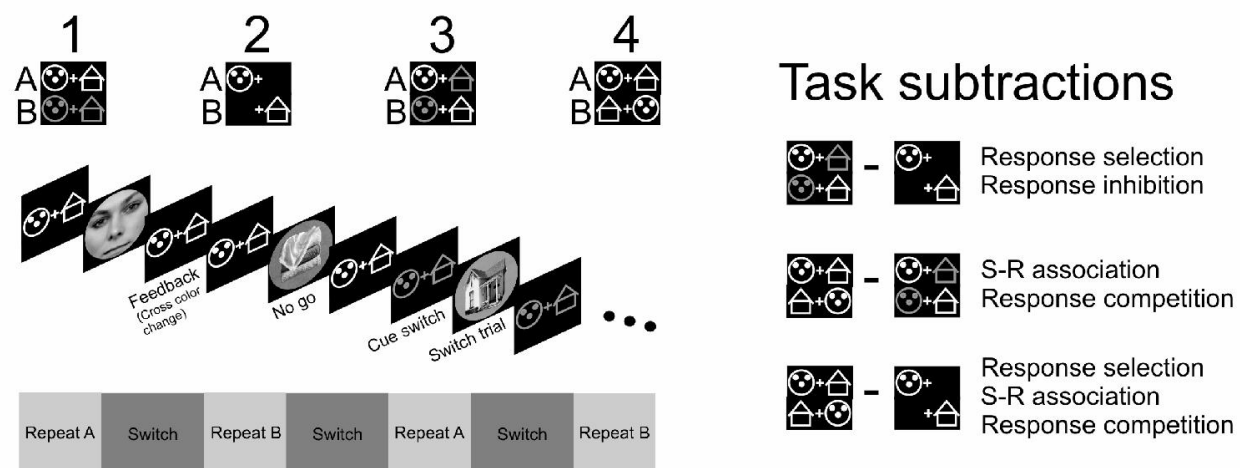

Figure 1 (next page): The fixation symbols seen by the subjects in each of the four switching tasks (in the upper panel, left), a typical trial sequence, illustrated for task 1 (in the middle panel, left), the large time-scale run-structure over all trials (in the lower panel, left), and the processes highlighted in three relative task comparisons by subtractive logic (right). The fixation-symbols in the left middle panel on the left are enlarged relative to the picture-stimuli for clarity.

We employed four switching visuomotor association tasks each requiring a different expression of component processes for correct performance (see Figure 1, left panel): a two-choice reaction time task switching between responding and passive viewing (task 1 ), a one-choice task switching between different non-competing stimulus-response (S$\mathrm{R}$ ) associations (task 2), a one-choice task switching between different S-R associations when ignoring previously salient stimuli (task 3), and a two-choice task switching between competing (reversed) S-R associations (task 4). All tasks contain 'no-go' trails. Task 1 singles out the additional processing for S-R association, and general response engagement and planning over passive viewing. Relative comparisons between task 2 , 3 , and 4 highlight the additional processes of interest in switching visuomotor association task (see Figure 1, right panel). Subtractive comparison of task 3 and 2 highlights activity or interactions dealing with response selection and response inhibition. Task 4 relies more than task 3 on processing of S-R associations and response competition. Finally, comparing task 4 and task 2 pinpoints networks involved in more complex S-R association, response selection and response competition. Within each of the tasks periods with many switches of S-R association rules (switch periods), consisting predominantly of 'switch' trials, were alternated with periods without any change in S-R association rules (repeat period), consisting predominantly of 'repeat' trials. These contiguous periods were used as the basis for the RFX GCMs, enabling inference on interactions specific to 'steady-state' repeat trials or dynamic updating of S-R associations within one task, and on the relative expression of interactions between tasks. 


\section{Methods}

\section{Subjects \& Task}

Sixteen subjects (aged 19-32 years, 8 females) performed four versions (referred to as Task 1-4) of a visual stimulus-response (S-R) association task in a changing context. The set of possible stimuli (Faces, Houses, and Objects) and the set of possible responses (Left and Right) were the same in the four versions of the task. In each version the context, which defined the correct response for every possible stimulus, switched continuously between two possible contexts, continuously changing the required $S-R$ associations. It is the pair of contexts, each a set of (different) $S-R$ associations, that defines each task (See Figure 1).

Object stimuli were present in each task and in every context as 'no-go' stimuli, never requiring a response. Task 1 switched between context $1 \mathrm{~A}$ (Faces-None, HousesNone, Objects-None) and context 1B (Faces-Left, Houses-Right, Objects-None). Thus, version 1 switched between a passive viewing context, and a response context in a twochoice setting. Task 2 switched between context 2A (Faces-Left, No House stimuli, Objects-None), and 2B (No Face stimuli, Houses-Right, Objects-None). Thus, Task 2 switched between two versions of a single- choice RT task, with a switch from one S-R association to the other, without conflicting stimuli (stimuli that are associated with a response in only one of the contexts). Task 3 switched between context 3A (Faces-Left, Houses-None, Objects-None) and context 3B (Faces-None, Houses-Right, ObjectsNone). Thus, version 3 also switched between two versions of a single-choice RT task, with a switch from one S-R association to the other, however, this time with conflicting stimuli in the same context. The fourth task version switched between context $4 \mathrm{~A}$ (Faces-Left, Houses-Right, Objects-None) and context 4B (Faces-Right, Houses-Left, Objects-None). Thus, version four switched between two two-choice contexts where the required responses continually conflict with those in the other context (through S-R reversal). To minimize memory confounds, the present context was continually signaled to the subject by symbols surrounding the fixation cross, that disappeared only briefly at presentation of a stimulus. A change in context was signaled by a change in fixation symbol.

In each version there were fifteen stimuli, five instances of each category Faces, Houses, and Objects. The stimuli were 8-bit grayscale photographs surrounded by a circular aperture that were shown briefly, for $133 \mathrm{~ms}$, with a stimulus onset asynchrony (SOA) varying from two to six seconds. At every response, feedback was given to the subject through a 500ms change in color of the fixation cross, green for a correct response, red for an incorrect one. The responses, a left or right index finger press, were recorded by fiber-optic button-boxes (LUMITouch, Photon Control, www.photoncontrol.com). All stimulus presentation, synchronization with volume acquisition of the MR-scanner, and recording of the responses was performed by Presentation (Neurobehavioral Systems, www.neuro-bs.com). The four tasks were implemented as fast event-related designs with a randomized stimulus onset asynchrony (SOA) of 2 to 6 seconds. A trial in any of the tasks was classified as a switch-trial if it was the first face or house stimulus after the switch-cue. All other face and house trials were designated repeat-trials. The SOA between cue change and the next trial was 3 to 7 seconds. In 
every task, periods with many cue changes (every 2 or 3 trials) consisting predominantly of switch trials, were alternated with periods with no cue change for 15 trials (predominantly repeat trials).

A fifth version of the task, formally equal to the fourth version, only with different stimulus categories (Animals instead of Faces, Tools instead of Houses), was also performed by each of the sixteen subjects but is not reported here. Each subject practiced each of the task-versions in the scanner, in the same order as in the experiment and immediately prior to the experimental runs in the same session. $A$ diffusion weighted measurement (not reported here) was performed while subjects were practicing. To further avoid practice effects and effects of habituation and weakening attention, half of the subjects performed the four task versions in reverse order. That is, the first and third quarter of the subjects (I and III) performed the task versions in the order 1-2-3-4, whereas the second and fourth quarter (II and IV) performed the task versions in the order 4-3-2-1. In addition, to exclude stimulus specific or response specific effects, all S-R associations in all contexts of the four task versions were reversed for half of the subjects. That is, the first two quarters of the subjects (I and II) performed all task versions as described above, the second two quarters (III and IV) performed all task-versions with Left and Right responses reversed from the above description.

\section{Image acquisition and analysis}

Images were acquired on a 3T whole body system (Siemens Trio, Erlangen, Germany) with a standard head coil. T2*-weighted functional images were acquired with a gradient echo $\mathrm{EPI}$ sequence $(\mathrm{TE}=30 \mathrm{~ms}, \mathrm{FA}=62 \mathrm{deg}$, $\mathrm{TR}=1000 \mathrm{~ms}, 64 \times 64$ matrix, FOV=224x224), with 17 obliquely oriented transverse slices ( $5 \mathrm{~mm}$ thick, $1 \mathrm{~mm}$ gap). A high-resolution anatomical reference volume was acquired for every subject with a 3D T1-weighted MPRAGE sequence (TE=4ms, 192×256x256 matrix, 1mm isotropic voxels). Each of the subjects performed each of the four tasks in a separate 540 time-point run of the scanner. One subject had to be excluded from analysis due to excessive motion during acquisition.

All data analysis was performed in BrainVoyager QX (Brain Innovation, Maastricht, the Netherlands). All anatomical volumes were transformed to the Talairach coordinate system (Talairach and Tournoux, 1988). The grey-white matter boundary was segmented using a semi-automatic intensity-based procedure. Subsequently, the cortical surface was reconstructed and inflated (Kriegeskorte and Goebel, 2001). To improve the spatial correspondence mapping between brains beyond Talairach space matching, the reconstructed cortices were aligned using curvature information reflecting the gyral/sulcal folding pattern. It has been shown that a cortical matching approach substantially improves statistical analysis across subjects by reducing anatomical variability (Fischl et al., 1999). The alignment of major gyri and sulci is achieved reliably by this method. Following (Fischl et al., 1999), the reconstructed cortical representation of each subjecthemisphere was morphed into a spherical representation suitable for across-subject nonrigid alignment. The curvature information computed in the folded representation is preserved and provides the matching criterion for the alignment process that minimizes 
the mean squared differences between the curvature of a source and a target sphere. The alignment proceeds iteratively following a coarse-to-fine matching strategy, from highly smoothed curvature maps to only slightly smoothed representations. The target for the alignment of each individual hemisphere is the dynamically updated average curvature map over all subjects. For each hemisphere, this cortex based alignment (CBA) method results in a vertex-by-vertex mapping from a standard spherical coordinate system (the standard sphere) to the individual subject hemisphere (the sphere-to-sphere mapping). Additionally, for each vertex on the subjects hemisphere, its position in the anatomical volume is known from the original folded cortex reconstruction, enabling data aligned to the anatomical volume to be mapped to the cortex based aligned hemisphere.

The time courses of activation of individual voxels were constructed from the functional images and corrected for the temporal difference in acquisition of different slices (slice scan time correction) using sinc interpolation. Subsequently, correction for bulk motion over time was corrected by rigid-body transformations, and linear trends and low frequency components (up to and including 4 cycles in the time course) were removed prior to any analysis. Voxel time courses were then co-registered to the structural volume using an affine transform and transformed into Talairach space and interpolated to a resolution of $3 \times 3 \times 3 \mathrm{~mm}$ using trilinear interpolation. No spatial or temporal smoothing was applied to the functional time courses. Recorded time-courses at the cortical grey matter were then resampled to the individually reconstructed cortical representation. The information from the cortex based alignment process was used to obtain a highly accurate subject-to-subject alignment of functional data time-courses on the cortical surface, suitable for group analyses. All analyses were also performed 3D Talaraich space to identify effects in subcortical structures.

Regional activations were analyzed using a fixed-effects General Linear Model (GLM) over all four tasks and all subjects that enabled between task (i.e. between run) inferences. Predictors for the cue-changes, control (object) stimulus, face stimulus and the house stimulus, were constructed as box-car functions and filtered through a linear model of the BOLD response (Boynton et al., 1996). Face and house predictors were further separated into switch-trials and repeat-trials. Statistical parametric maps (SPMs) were computed for the contrast of face and houses against objects, and switch trials against repeat trials for each task, and for contrasts of faces and houses between task 2 , 3 , and 4 . The between run comparisons were confined to these tasks because task 1 is set apart from 2, 3, and 4, since it does not involve the switching between two non-trivial $\mathrm{S}-\mathrm{R}$ associations. Maps were thresholded at an expected false discovery rate (FDR) of $\mathrm{q}$ $<0.05$ (Genovese et al., 2002), or at a T-value of 2, applying a cluster threshold.

Individual Granger Causality Maps (GCM) were computed for a given reference ROI as detailed in (Roebroeck et al., 2005). The influence measures $\mathbf{F}_{\mathbf{x} \rightarrow \mathbf{y}}, \mathbf{F}_{\mathbf{y} \rightarrow \mathbf{x}}$, and $\mathbf{F}_{\mathbf{x} \cdot \mathbf{y}}$ (reflecting Granger causality from $x$ to $y$, Granger causality from $y$ to $x$ and instantaneous correlation, respectively) were computed for every voxel from average time-course of the voxels in the reference ROI (as $\mathbf{x}$ ) and the voxel time-course (as $\mathbf{y}$ ). In accordance with (Roebroeck et al., 2005), the influence difference term $\left(\mathbf{F}_{\mathbf{x} \rightarrow \mathbf{y}}-\mathbf{F}_{\mathbf{y} \rightarrow \mathbf{x}}\right)$ was then computed for every voxel to form the difference-GCM (dGCM), mapping influence to and from the $\mathrm{ROI}$ over the brain. Activation foci from the GLM-analysis were used as reference 

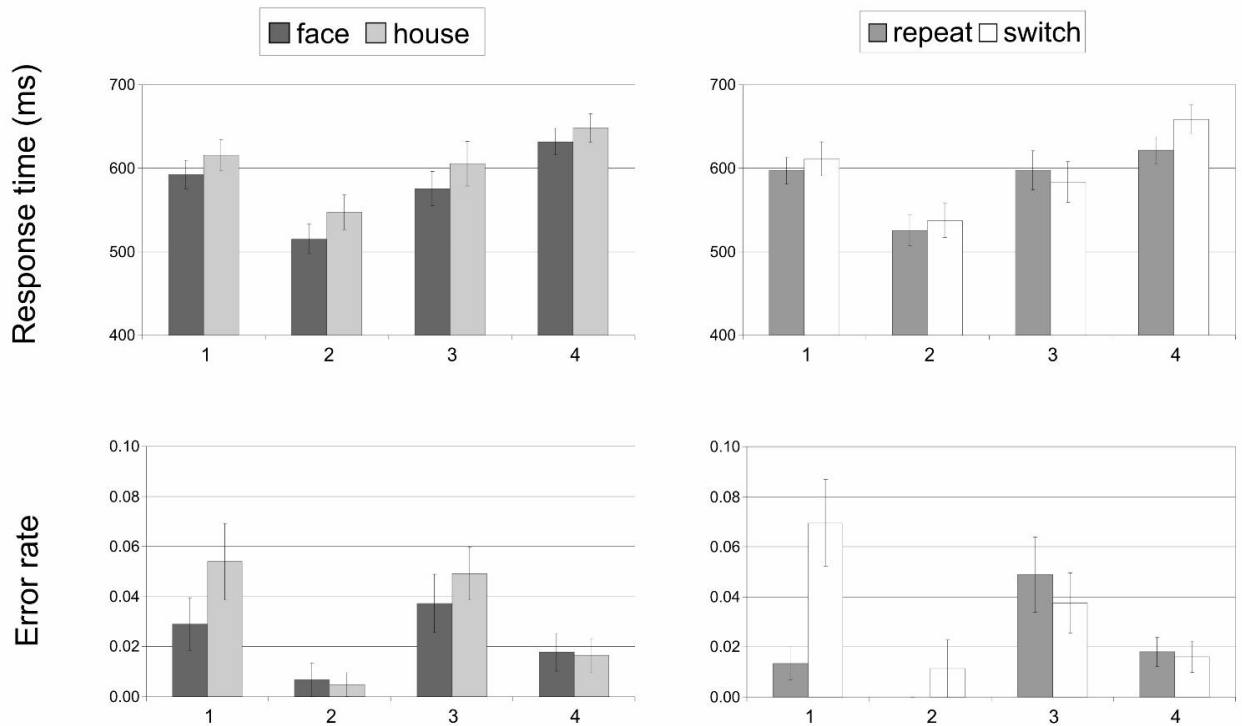

Figure 2: Average reaction times (upper panels), and error rates (lower panel), split for face and house stimuli (left panels) and repeat and switch trials (right panels). Error bars represent standard errors.

regions for each subject to compute dGCMs in the different tasks (1 to 4) and contexts (switch period vs repeat period). The order of the autoregressive models used for computation of the influence measures was set to 1. Random effects (RFX) dGCMs at the group level were computed by a summary statistics approach, performing second level one-sample t-tests using the variance over single-subject dGCMs at a given voxel or vertex.

Significant modulation of effective connectivity by context (switch-period vs repeat period) or task-version was assessed using second level two-sample t-tests. In this case, the difference in mean value over subjects between the dGCMs computed for each of the contexts or tasks was tested. No corrections for multiple comparisons were applied to the RFX GCMs. All RFX GCMs (t-maps) were thresholded at $p<0.05$. All maps are visualized on an inflated average cortical surface that shows common curvature over all subjects after cortex based alignment. All RFX GCMs were smoothed on the surface for visualization purposes.

\section{Results}

\section{Behavioral results}

Response times (RTs) and error rates (ERs) were each separately analyzed in a $4 \times 2 \times 2$ repeated-measures analysis of variance. The three factors were: task $(1,2,3$, or 4$)$, trial 
(switch or repeat), and stimulus (face or house). RT analysis was performed only on correct responses, ER analysis was performed only on errors at response-stimuli (face and house), i.e. not on responses at object stimuli and missed responses at faces or houses.

In the RT data (see Figure 2, upper panels), the effects of task $[F(3,12)=23.105, p$ $<0.001]$, stimulus $[F(1,14)=16.650, p<0.002]$, and trial $[F(1,14)=5.166, p=0.039]$ on RT were all significant. In addition, there was a significant interaction between factor task and trial $[F(3,12)=9.123, p<0.003]$.

Post-hoc test showed the main effect of tasks to be caused by significant RT differences between tasks, except between task1 and 3 (see Figure 2, upper panels). The main effect of stimulus was due to subjects responding faster to faces $(578.9 \mathrm{~ms})$ than to houses $(604.4 \mathrm{~ms})$ over all tasks. The interaction between task and trial is explained by faster responses to repeat trials $(621.5 \mathrm{~ms})$ than to switch trials $(658.7 \mathrm{~ms})$ in task 4 , without significant differences in task 1, 2, and 3.

In the ER data (see Figure 2, lower panels), only the effect of task $[F(3,12)=7.506$, $p<0.005]$ was significant. There was no effect of stimulus $[F(1,14)=2.902, p=0.111]$ or of trial $[F(1,14)=2.417, p=0.142]$ on ER. Additionally, the interaction between task and trial was marginally significant $[F(3,12)=3.115, p=0.067]$. This pattern of effects was due to considerably more errors made by the subject on the switch trials of task 1 than in the other tasks. Furthermore, on average more errors were made at the response stimuli in task 1 and 3 than task 2 and 4 (see Figure 2, lower panels). To check whether the higher error rates at switch trials in task 1 and task 3 could be due to the occurrence of failure-of-inhibition errors (pressing at a face or house when a response is no longer required), post-hoc frequency analysis was performed. Indeed, 16 out 18 errors (over all subjects) in task 1, and all 35 errors in task 3 were failure-of-inhibition errors.

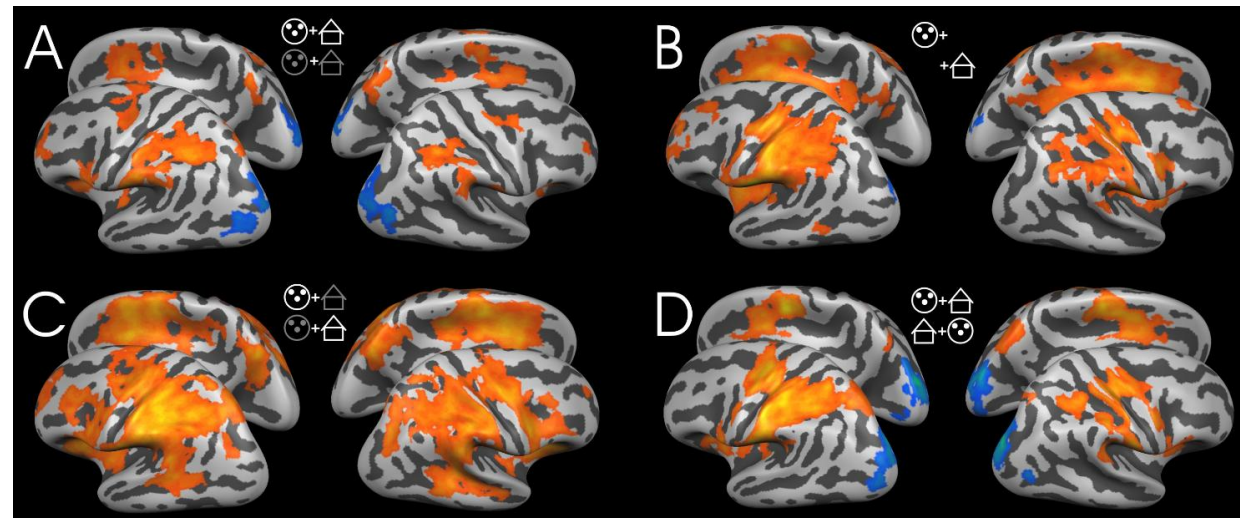

Figure 3: GLM activation for contrast of face\&house vs object (in orange to yellow), and object vs face\&house (in blue to green) for task 1 in $A$, task 2 in B, task 3 in C, and task 4 in D. (FDR: q .05). 


\section{GLM-analysis}

GLM analysis focused mainly on identifying the brain regions involved in possible component processes for the switching visuomotor association tasks, using the task comparisons in Figure 1. First, relative activation of response stimuli (faces and houses) compared to no-go stimuli (objects), and relative activation of switch trials compared to repeat trials was investigated to identify the brain-regions primarily involved in the tasks. Activation for a contrast of face \& house (response stimuli) against objects (control stimulus) for tasks 1 to 4 is shown in Figure 3, thresholded at an FDR of $q<0.05$. Over all tasks, higher activation for objects can be seen in occipital, occipito-temporal, and occipito-parietal areas. Higher activation for faces and houses is mostly present in inferior and superior parietal areas, and lateral and medial prefrontal areas. This highlights the important role of fronto-parietal systems in the switching visuomotor association tasks.

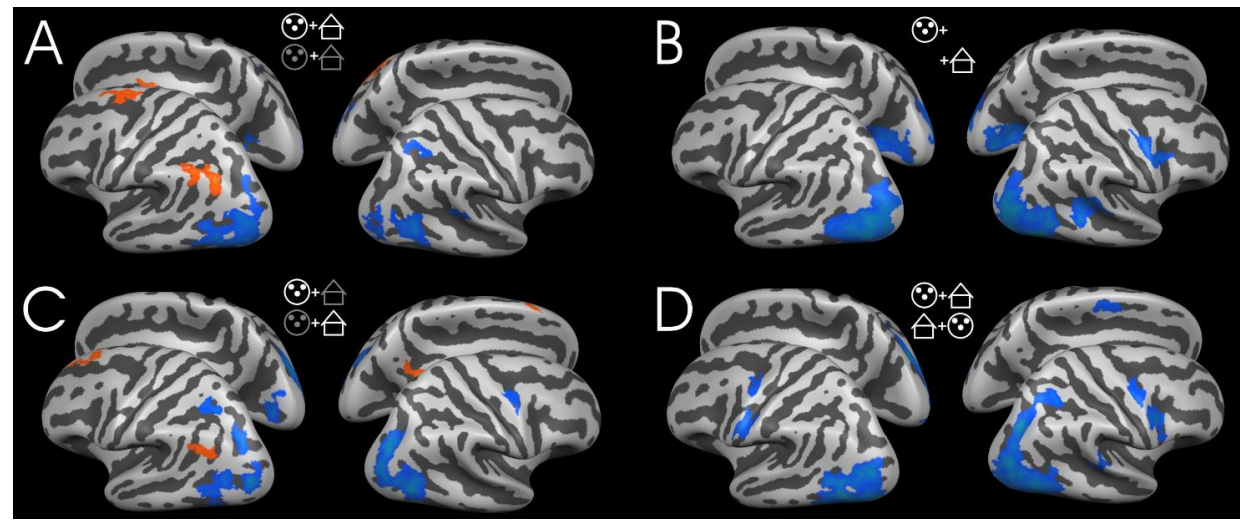

Figure 4: GLM activation for contrast of switch > repeat (in orange to yellow), and repeat $>$ switch (in blue to green) for task 1 in $A$, task 2 in $B$, task 3 in $C$, and task 4 in D. (t > 2, cluster > 50mm2).

In Figure 4, a contrast is shown for switch trials against repeat trials for all four tasks, thresholded at $t>2$, no correction for multiple comparisons. Activity for repeat trials is generally higher over all tasks in occipito-temporal and occipito-parietal areas, and in some regions of superior parietal and lateral prefrontal cortex. Activation for switch trials relative to repeat trials is very sparse in the present tasks. Some activation can be found in left inferior parietal and dorsal prefrontal cortex.

Investigation of the systems involved in the various sub-processes of the tasks using the described tasks contrasts between tasks 2, 3, and 4 are shown in Figure 5. A comparison of response stimuli in task 3 and task 2 shows greater activation in dorsal and ventral premotor cortex, and in occipito-parietal and posterior parietal areas, 
primarily in the left hemisphere. Thus, posterior parietal cortex and dorsal premotor cortex are shown to be activated in a contrast highlighting response selection and response inhibition. A direct comparison between response stimuli in tasks 4 and 3 shows greater activity in task 4 in superior parietal cortex, dorsal premotor cortex, and the (pre)SMA, involving these in $S-R$ association and processing of response competition. Finally, a paired contrast for additional activity for task 4 stimuli relative to task 2 stimuli shows activation in bilateral dorsal premotor areas, both anterior and posterior SPL areas as well as some occipito-parietal regions, (pre)SMA and left-side ventrolateral prefrontal cortex, involving these regions in response selection, S-R association and/or response competition. Overall, these contrasts again show a high involvement of primarily left localized fronto-parietal systems in these visuomotor association tasks. Within these, dorsal premotor cortex seems to be selectively involved in response selection and response competition. Furthermore, a tendency towards dissociation of left superior parietal areas is shown, with posterior superior parietal cortex associated with response selection processes, whereas anterior superior parietal cortex seems to be involved in S-R association. These three left-hemisphere regions were therefore chosen as reference ROls for RFX GCM analysis to investigate their interactions with other brain regions involved in the performance of the tasks. 

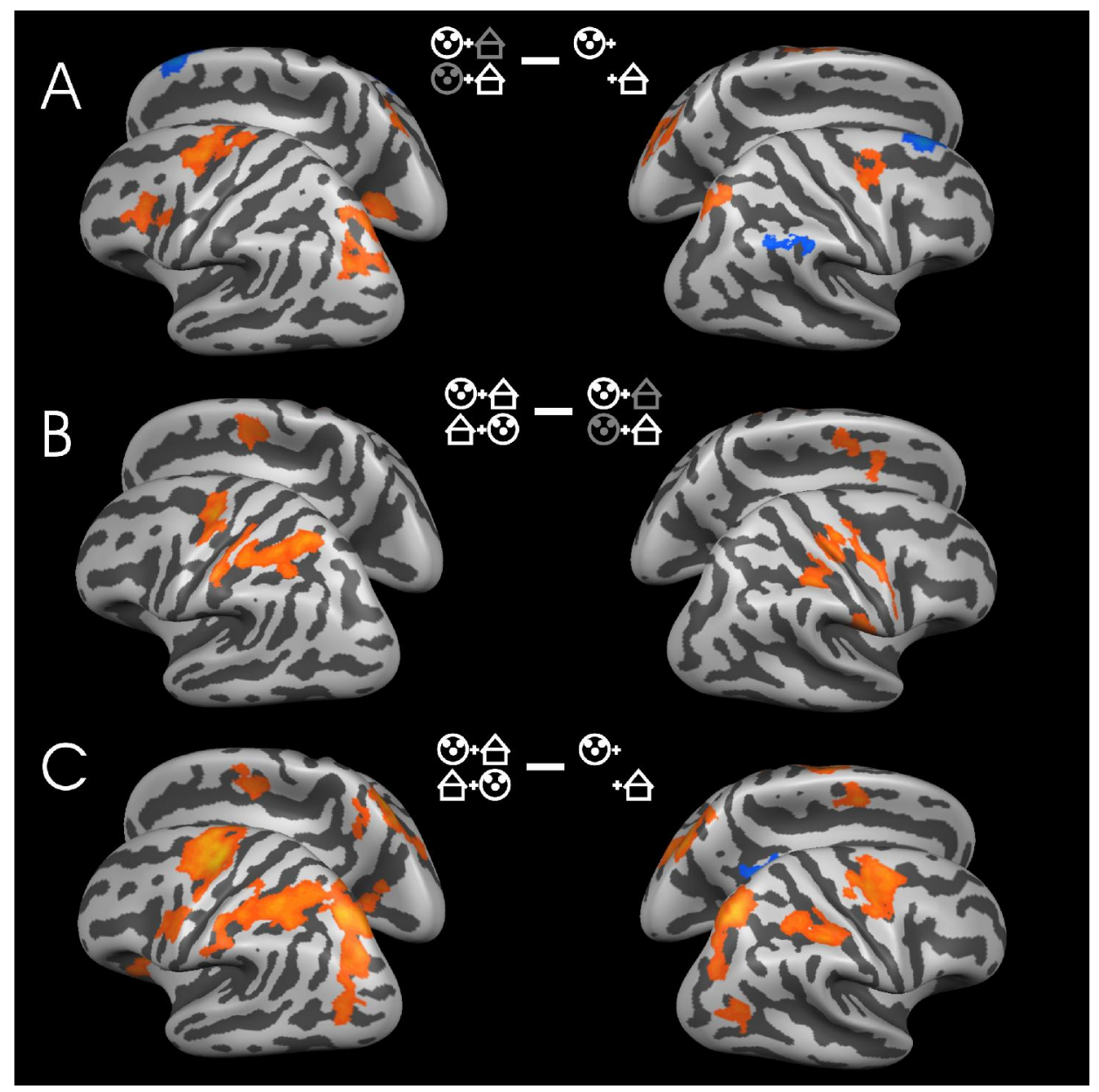

Figure 5: A) GLM activation for contrasts of face3\&house3 > face2\&house2 (in orange to yellow) and face2\&house2 > face3\&house3 (in blue to green). B) GLM activation for contrasts of face4\&house $4>$ face $3 \&$ house 3 (in orange to yellow) and face3\&house $3>$ face 4 \&house 4 (in blue to green). C) GLM activation for contrasts of face4\&house4 $>$ face2\&house2 (in orange to yellow) and face $2 \&$ house $2>$ face $4 \&$ house 4 (in blue to green). ( $t<2$, cluster $>50 \mathrm{~mm} 2$ ).

Figure 6 (next page): Random effects Granger causality maps (RFX GCMs) for a reference region in left dorsal premotor cortex (marked in red) for all four tasks. The upper panel for each task contains the RFX GCM for the 'switch' task periods, the lower panel contains the RFX GCM for the 'repeat' task periods. Source areas influenced by the reference region are marked in blue, target areas influencing the reference area are marked in green. Significant differences in influence at the random effects level between 'switch' and 'repeat' task periods are marked in orange to yellow and light-blue to green. Insets show the RFX GCM in a transverse slice (at Talaraich z-coordinate) to show significant differences between 'switch' and 'repeat' periods in subcortical structures, if present. 


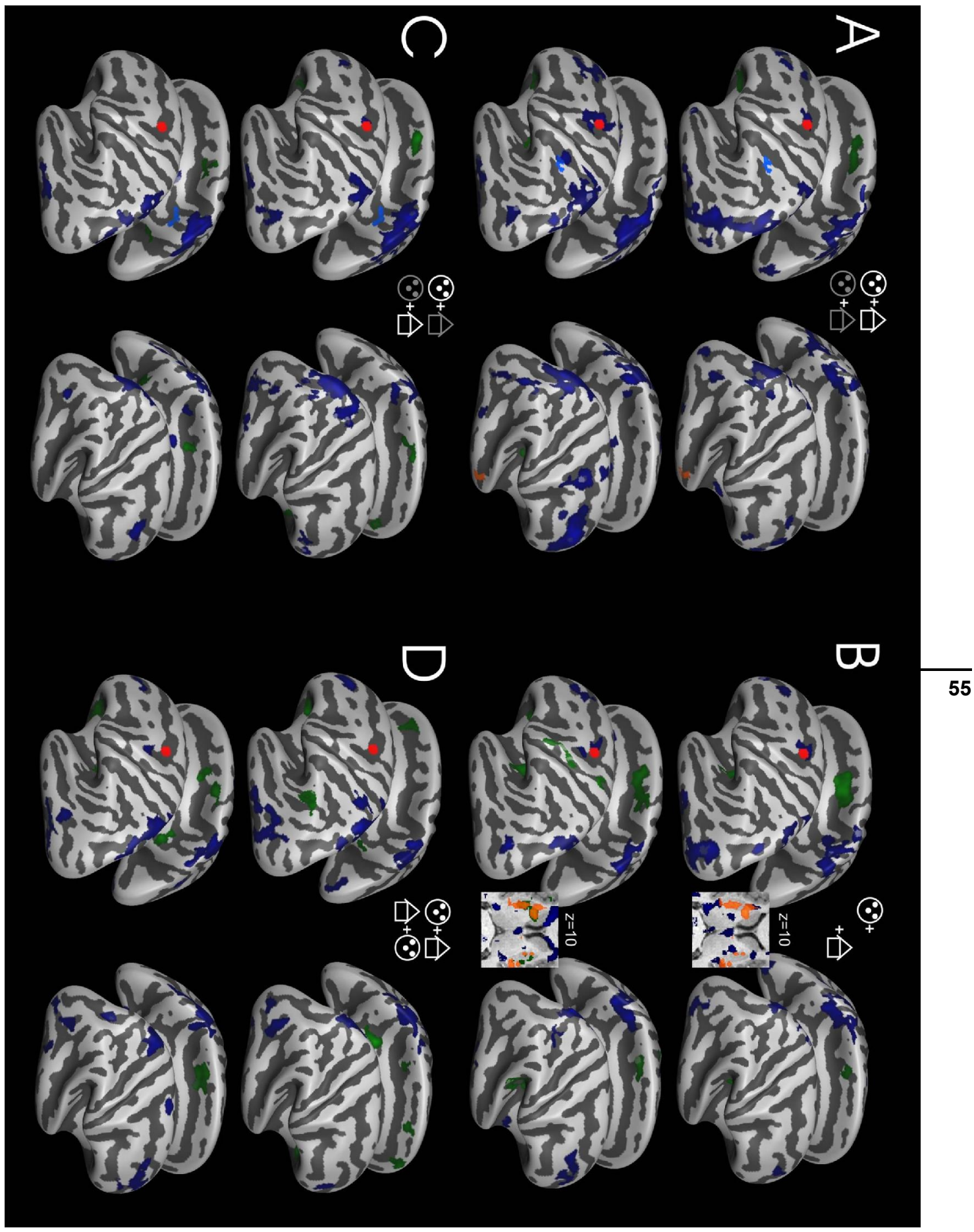




\section{GCM-analysis}

Figure 6 shows RFX dGCMs for the reference region in the left dorsal premotor (PMd) cortex that was shown to be more activated in tasks 3 and 4 than in task 2 (see Figure 5). Over all tasks the main target of output from the left PMd seems to be the superior lateral and medial parietal cortex. Additional output targets are the lateral occipital cortex and anterior prefrontal cortex. The main sources of input to the left PMd are seen to be SMA/preSMA, the anterior insula, and to a lesser degree, left side ventral premotor areas (in task 2) and inferior parietal areas (in task 4). The differences in influence between switch periods and repeat periods are very modest overall, indicating the role of the dorsal premotor cortex in its interactions with other systems is very similar in switch trials and repeat trials. The only clear contextual modulation is input from the putamen in repeat periods but not switch periods of task 2 .

Figure 7 (next page): Random effects Granger causality maps for a reference region in left anterior SPL (marked in red) for the 'switch' periods of task 2 in A, task 3 in B, and task 4 in C. Significant differences in influence at the random effects level between 'switch' and 'repeat' task periods are marked in orange to yellow and light-blue to green. Insets show the RFX GCM in a transverse slice (at Talaraich z-coordinate) to show significant differences between 'switch' and 'repeat' periods in subcortical structures, if present. D) Significant differences in influence at the random effects level between 'switch' periods of task 3 and task 2 marked in orange to yellow and light-blue to green, overlaid on the RFX GCM for the switch period of task 3. E) Significant differences in influence at the random effects level between 'switch' periods of task 4 and task 3 marked in orange to yellow and light-blue to green, overlaid on the RFX GCM for the switch period of task 4. F) Significant differences in influence at the random effects level between 'switch' periods of task 4 and task 2 marked in orange to yellow and light-blue to green, overlaid on the RFX GCM for the switch period of task 4 . $(\mathrm{t}<0.05$, cluster $>20 \mathrm{~mm})$. 

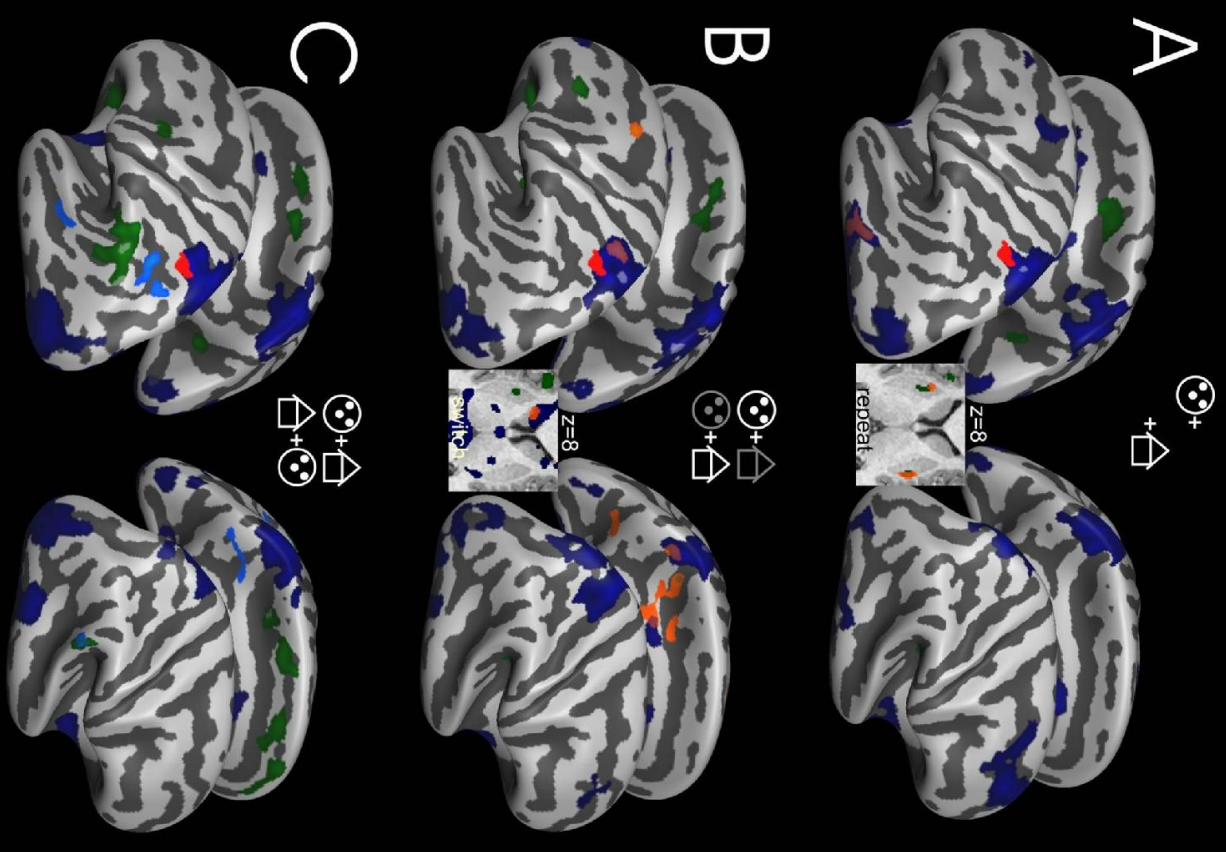

7
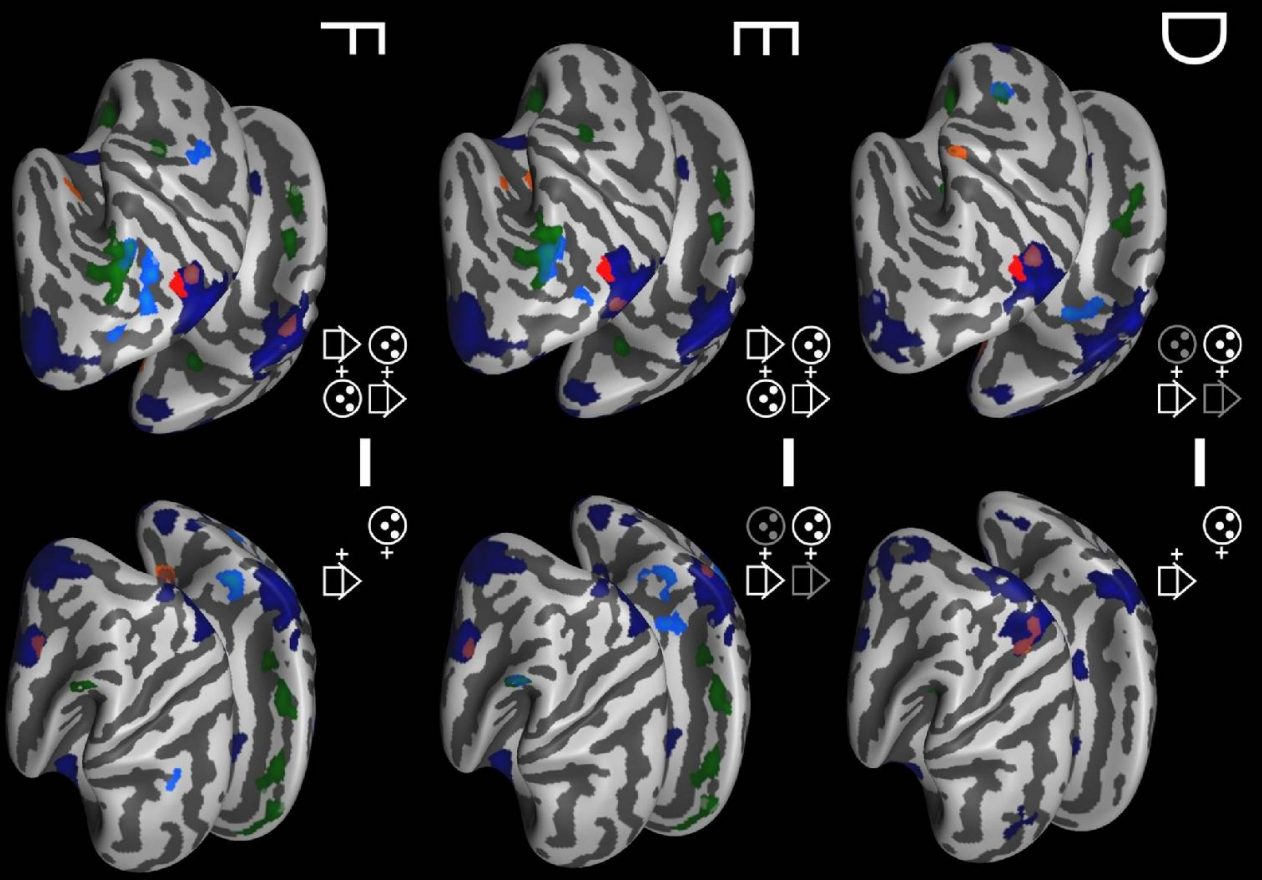
RFX dGCMs for a reference ROI in the left anterior superior parietal lobule (SPL), more active in task 4 than in tasks 2 and 3 (see Figure 5), are shown in Figure 7. The RFX GCMs are shown for tasks 2, 3, and 4, and for the important subtractive comparisons between them. Over tasks 2, 3, and 4 the main target areas of output from the left anterior SPL are bilateral inferior temporal and occipito-temporal areas, and more superior and medial parietal areas. The main sources of input to the left anterior SPL are medial prefrontal areas (SMA/preSMA), left ventrolateral prefrontal areas and anterior insula, and (mainly in task 4) the left inferior parietal lobule (IPL). The left anterior SPL shows some interesting modulation of its interactions with other areas by task context (switch periods vs. repeat periods). In task 2, an additional influence in switch periods from the left anterior SPL to the left inferotemporal cortex can be seen. Furthermore, a significant increase in the influence from the bilateral putamen can be seen in the repeat periods. In task 3 increased influence to other superior and medial parietal areas and to the left-side caudate nucleus is evident during switch periods. In task 4 an increased input from inferior parietal areas during switch periods is evidenced. Direct comparisons between the switch periods of tasks 2, 3, and 4 show the different interactions of the left anterior SPL with other areas over the three tasks. A direct comparison of the switch periods in task 3 and 2 shows additional output from left anterior SPL to left and right superior parietal cortices, and input from left ventrolateral prefrontal cortex in task 3 compared to task 2 . Comparing task 4 and 3 reveals mainly an increase in input to the left anterior SPL from inferior parietal areas in task 4. Finally, the differences in interaction of the left anterior SPL between task 2 and 4 are seen to be largely a union of those between 2 and 3 and between 3 and 4: larger output to superior parietal areas and larger input from both left inferior parietal and left lateral prefrontal areas in task 4.

Figure 8 (next page): Random effects Granger causality maps for a reference region in left posterior SPL (marked in red) for the 'switch' periods of task 2 in A, task 3 in B, and task 4 in C. Significant differences in influence at the random effects level between 'switch' and 'repeat' task periods are marked in orange to yellow and light-blue to green. Insets show the RFX GCM in a transverse slice (at Talaraich z-coordinate) to show significant differences between 'switch' and 'repeat' periods in subcortical structures, if present. D) Significant differences in influence at the random effects level between 'switch' periods of task 3 and task 2 marked in orange to yellow and light-blue to green, overlaid on the RFX GCM for the switch period of task 3. E) Significant differences in influence at the random effects level between 'switch' periods of task 4 and task 3 marked in orange to yellow and light-blue to green, overlaid on the RFX GCM for the switch period of task 4. F) Significant differences in influence at the random effects level between 'switch' periods of task 4 and task 2 marked in orange to yellow and light-blue to green, overlaid on the RFX GCM for the switch period of task 4 . $(\mathrm{t}<0.05$, cluster $>20 \mathrm{~mm})$. 

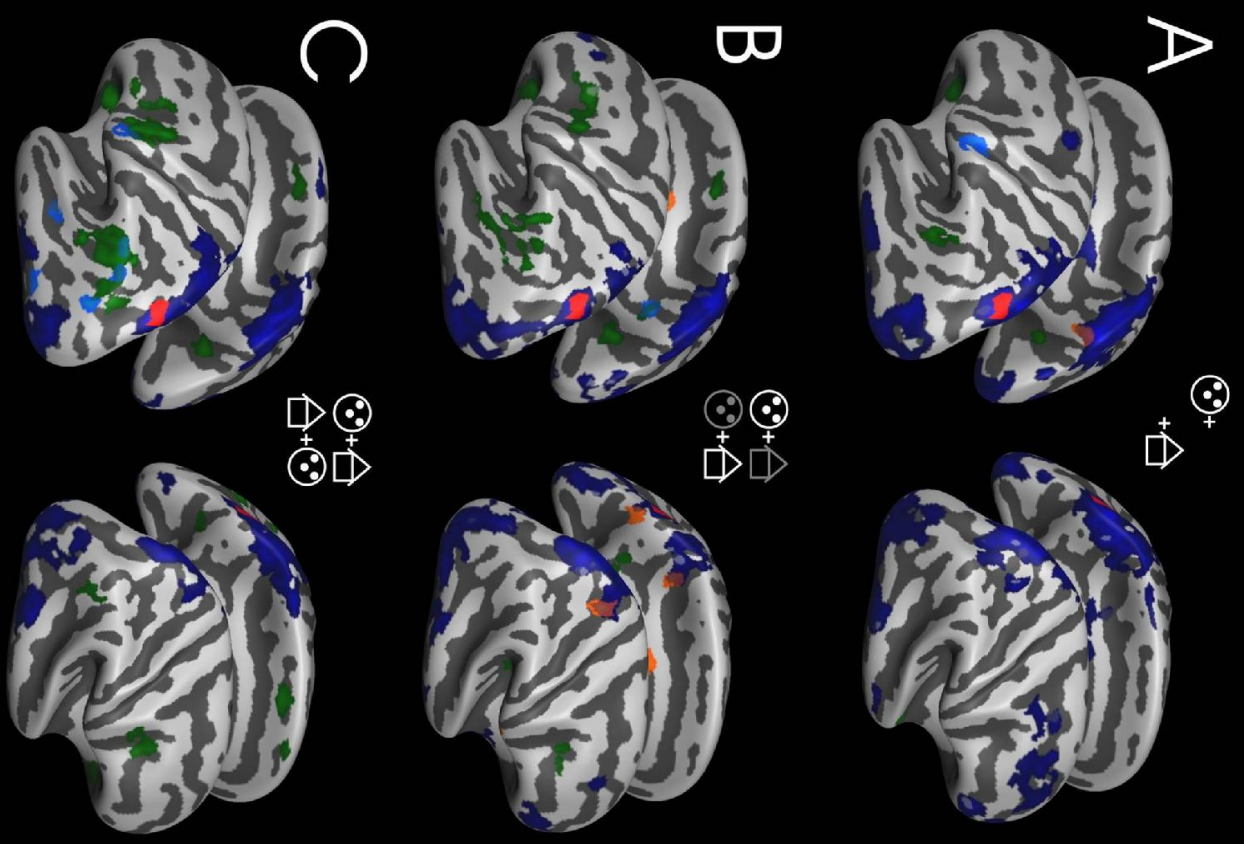

$\Pi$
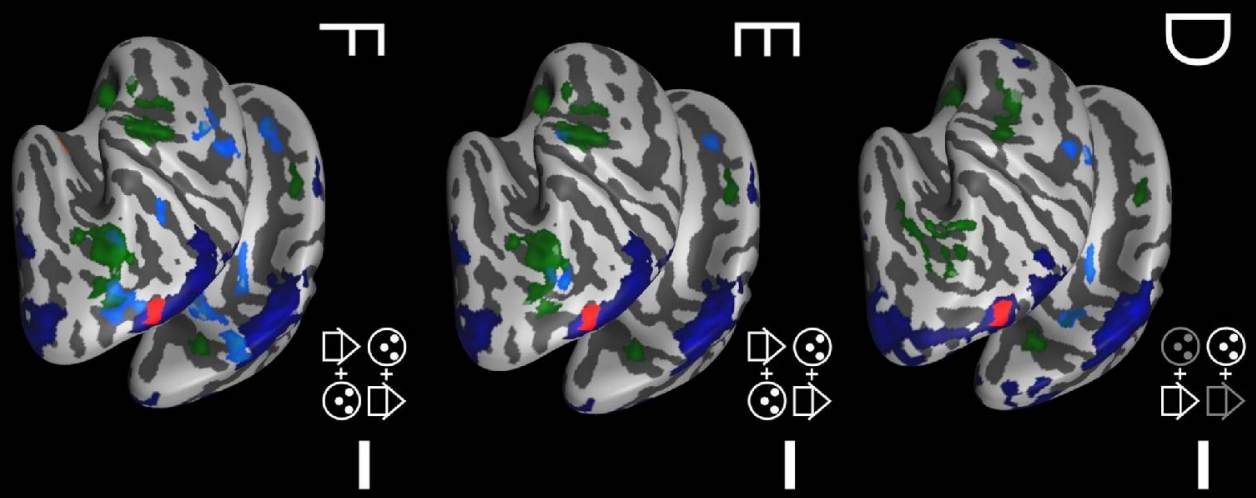

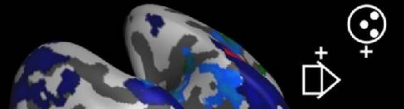
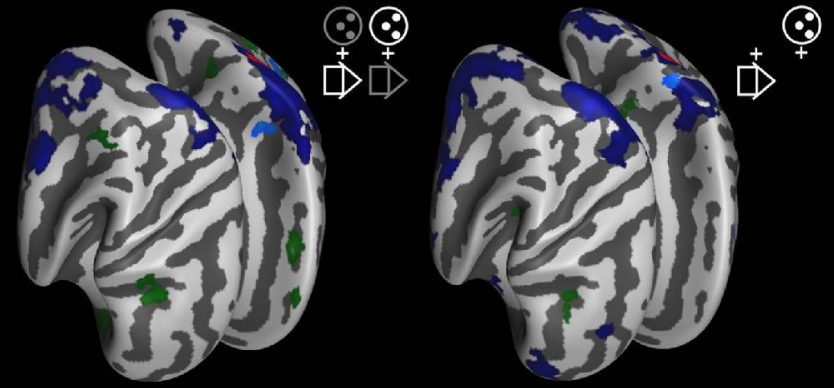


\section{Discussion}

Behavioral results show that the intended experimental manipulation were successful. The four tasks differ in their average reaction times (RTs), supporting the hypothesis that different cognitive processes are involved in their successful performance. Task 2, a basic one-choice RT task (with an additional no-go stimulus, the object) switching between two different target stimuli with different responses, was performed the fastest. RTs for tasks 1 and 3 were slower than those for task 2 , but not significantly different from each other. RTs for task 1 originate only from its response phases (it switched between responding and passive viewing). The longer RTs for task 1, a two-choice RT task with no-go stimulus, are probably due to a larger amount of attended stimuli (faces and houses), more responses that must be prepared, and a larger amount of S-R mappings that have to be kept online. The longer RTs in task 3 , relative to task 2 , are presumably caused by additional inhibitory selection processes for S-R associations to be ignored, and increased readiness and attentional demands to react only to current imperative stimuli. Finally, task 4 , a switching two-choice RT task with conflicting reversed S-R associations was performed the slowest on average. Presumably, the required additional processes of $S-R$ remapping and response conflict detection and reduction caused this increase in RTs. Over all tasks, switch cost was very low and nonsignificant, except in task 4 . This reflects the design of the experiment in which pre-cues preceded S-R contingency switches with a long (3-7 second) pre-delay. Behavioral studies have shown that switch costs reduce in such circumstances and that only modest 'residual switch costs' remain in this context (Monsell, 2003). The low switch costs show that subject have been able to effectively prepare the new S-R mapping at the cue-switch, such that performance degradation at following response trials is minimal. Over all tasks, an additional stimulus-specific effect was observed: the response to face-stimuli was faster than that to house-stimuli. Faces are a more natural and highly trained perceptual category than houses. Furthermore, in picture stimuli faces generally contain less variation in low level characteristics than house pictures. The effect is thus likely to be a fully perceptual effect which, importantly, did not interact with the task and trial (switch or repeat) manipulations.

GLM analysis localized the brain systems involved in the performance of the four tasks. Comparing face \& house (response stimuli) against objects (control stimulus) shows that, over all tasks, higher activation for objects can be seen in occipital, occipitotemporal, and occipito-parietal areas, whereas higher activation for faces and houses is mostly present in inferior and superior parietal areas, and lateral and medial prefrontal areas. This stresses the important role of fronto-parietal systems in the switching visuomotor association tasks, consistent with previous studies (Cools et al., 2004; Crone et al., 2006; Dove et al., 2000; Shafritz et al., 2005). Despite a modest behavioral switch cost over the four tasks, with RTs for switch trials slightly slower (12 ms on average) than those for repeat trials, activity for repeat trials is generally higher over all tasks in occipito-temporal and occipito-parietal areas, and in some regions of superior parietal and lateral prefrontal cortex. Tendency toward higher activity for switch trials could only be found in left inferior parietal and dorsal premotor cortex. Other studies have also found switch-related activity in inferior parietal and superior temporal cortex (Barber and Carter, 2005; Smith et al., 2004), as well as in (ventro)lateral prefrontal cortex (Braver et 
al., 2003; Cools et al., 2004; Crone et al., 2006; Shafritz et al., 2005; Smith et al., 2004), and SMA/preSMA (Crone et al., 2006; Rushworth et al., 2002; Smith et al., 2004). Generally, the surplus activity for switch-trials in these studies tended to be larger than observed here. A possible explanation can be found in differences of task design. The current study was designed to keep overhead processing at switch-trials limited to components specifically dealing with response selection \& competition, and reconfiguration of $\mathrm{S}-\mathrm{R}$ contingencies. To that end, meaningful pre-cues signaling ruleswitches (the fixation symbols) rather than arbitrary symbols (e.g. diamonds, squares) were used at long pre-delays (3-7 seconds). Indeed, behavioral switch-costs were minimal, supporting the active successful preparation of changing S-R contingencies in the subjects at switch-cues. Furthermore, activity for switch-cues was found to be large and extensive in fronto-parietal brain areas (not shown), highlighting the network of areas involved in the active preparation of changing attentional focus, motor responses, and S-R mappings needed to correctly respond (or inhibit responses) to oncoming stimuli.

\section{Left dorsal premotor cortex}

The task comparisons in Figure 1 were used to identify the brain regions involved in possible component processes for the switching visuomotor association tasks. A comparison of response stimuli (faces and houses) in task 3 and task 2 shows greater activation in dorsal premotor cortex (PMd), occipito-parietal and medial parietal areas, primarily in the left hemisphere. The primary difference between task 2 and task 3 is the presence of additional response inhibition and selection processes in task 3 . Stimuli have to be ignored that in an earlier S-R context needed a response, requiring inhibition processes. Similarly, after a switch-cue the same stimuli require a response again, necessitating active response selection and execution. Previous investigations into S-R association tasks have shown the dorsal aspects of lateral premotor and prefrontal cortex to be involved in response selection among multiple competing responses (Bunge, 2004; Bunge et al., 2002; Cavina-Pratesi et al., 2006; Rushworth et al., 2002) and preparation of likely movements (Cavina-Pratesi et al., 2006; Thoenissen et al., 2002). An explicit investigation into response inhibition (Wager et al., 2005) found premotor cortex to be among a set of areas engaged in response inhibition over multiple tasks, along with the anterior insula, anterior prefrontal, dorsolateral and inferior parietal areas. The activation of PMd in a contrast of response stimuli in task 2 and task 3 seems in line with these findings, affirming its role in processes of response selection and inhibition.

RFX GCM analysis with the left PMd as the reference region identified its interactions with other cortical and subcortical systems in switching visuomotor association tasks. The main target of output from the left PMd is the superior lateral and medial parietal cortex. The main sources of input to the left PMd are SMA/preSMA and the anterior insula. Previous investigations into switching visuomotor association tasks have shown SMA/preSMA to be involved in overcoming interference from the previously activated task set (Crone et al., 2006) and selecting between response sets at a superordinate level (Rushworth et al., 2002). Indeed bilateral SMA/preSMA was 
activated in both the task 3 vs. task 2 contrast and the task 4 vs. task 3 contrast involving it in response competition and selection processes. Together these results seem to imply a close connection between medial (pre)SMA and lateral PMd in the selection of responses, specifically in situation where conflict between competing responses has to be resolved. The medial (pre)SMA (perhaps in conjunction with Anterior Cingulate Cortex (Carter et al., 1998)), could resolve conflict at the level of response sets or even sets of S-R associations and select responses at a superordinate level (Rushworth et al., 2002). The results of this processing could then be used to influence processes in the lateral PMd to select individual response on a trial-to-trial basis. Perhaps this influence can be understood as a top-down signal that biases the competition between response representation at a lower level (Desimone and Duncan, 1995; Miller and Cohen, 2001; Yeung et al., 2006). The differences in influence from and to the left PMd between switch periods and repeat periods are very modest overall, indicating the role of the dorsal premotor cortex in its interactions with other systems is very similar in switch trials and repeat trials. That would be compatible with a role in trial-to-trial selection of individual responses, which has to take place both in switch trials and repeat trials. The only clear contextual modulation is input from the putamen in repeat periods but not switch periods of task 2. In earlier studies using visuomotor associations, the striatum was found to be involved in transformation of concrete stimulus exemplar information into motor responses (Cools et al., 2004; Crone et al., 2006), while also playing a role in the inhibition of task inappropriate responses and the execution of task appropriate responses (Shafritz et al., 2005) and overriding previous S-R association when switching between tasks (Crone et al., 2006). In these roles the striatum would certainly be another source of important information for the selection of responses in the dorsal premotor cortex. The fact that its influence on activity of the left PMd was found to be more extensive in the repeat periods of task 2, were a single unambiguous response is associated with a single stimulus category, might indicate that its modulatory input is particularly important when specific $S-R$ associations are made in a non-conflicting context.

Input from the anterior insula is seen for both the dorsal premotor and superior parietal regions investigated here. Furthermore, its input seems rather constant over switch and repeat periods of all tasks. The anterior insula is known to be anatomically connected with limbic system structures (specifically the amygdala). Thus, its tonic influence over extended periods could signify the input from evaluative processes in the limbic system and the influence of the emotional and motivational state of the subject.

\section{Left anterior superior parietal lobule}

Comparing between response stimuli in tasks 4 and 3, to identify areas involved in S-R association and response competition, shows greater activity in the left anterior superior parietal lobule (SPL) in task 4 . The main differences between task 3 and task 4 are the greater number of $\mathrm{S}-\mathrm{R}$ associations kept active and the conflicting responses in the different $S-R$ association rules in task 4 , since $S-R$ contingencies have to be reversed. Earlier investigations into S-R association tasks have shown the left SPL to be involved in preparing/keeping online a range of candidate responses in the current task context 
(Bunge et al., 2002; Thoenissen et al., 2002), and in activating and controlling sets of S$\mathrm{R}$ associations (Brass and von Cramon, 2004; Braver et al., 2003; Cavina-Pratesi et al., 2006; Crone et al., 2006). The activation of left SPL in a contrast of task 3 and task 4 response stimuli is well inline with these studies, and compatible with the idea that left SPL is more engaged when more (possibly conflicting) S-R associations have to be kept online. RFX GCMs with the left anterior SPL as a reference region showed its main targets of output to be the bilateral inferior temporal and occipito-temporal areas, and more superior and medial parietal areas. Occipito-temporal and inferior temporal cortex are well know to be engaged in the higher order processing of visual information resulting in recognition and categorization of elements in visual stimulus arrays. Discrete areas in inferotemporal cortex have been identified that are involved in the identification of specific visual stimulus categories, such as the Fusiform Face Area (FFA) for faces (Kanwisher et al., 1997), and the Parahippocampal Place Area (PPA) for places or houses (Epstein and Kanwisher, 1998), involved in the processing of the visual stimuli used in the current tasks. An important part of the role of the left anterior SPL in activating and controlling sets of S-R associations could be the top-down attentional modulation of visual processing to bias processing for the relevant stimulus categories given the currently active S-R association. In this sense the well-established role of the SPL in visual attention (Colby and Goldberg, 1999) complements and supports its putative additional role of controlling S-R associations. Furthermore, the influence from left anterior SPL to inferotemporal cortex was see to be statistically different in switch and repeat periods in task 2 . There is a larger amount of influence from the left anterior SPL to the left inferotemporal cortex in switch periods than in repeat periods. This would seem to reflect to high demand on top-down attentional control in switch periods in task 2 , where a different stimulus has to be detected and responded to in the two S-R contexts.

The main sources of input to the left anterior SPL are medial prefrontal areas (SMA/preSMA), left ventrolateral prefrontal cortex (VLPFC) and anterior insula, and (mainly in task 4) the left inferior parietal lobule (IPL). In previous investigation into S-R association tasks, the left vLPFC has been shown to be involved in processing, keeping online, and updating the relevant task set (Brass and von Cramon, 2004; Braver et al., 2003; Bunge, 2004; Crone et al., 2006; Shafritz et al., 2005). The activation of left vLPFC in a contrast of task 2 and 4 response stimuli is in line with these studies, since task-set processing and updating is more engaged in task 4, where two conflicting task-sets of two S-R associations have to be switched, than in task 2, where two non-conflicting simpler task-sets of one S-R association have to be used. Thus, the influence from left VLPFC to left anterior SPL seems to underlie a form of high-level control where superordinate level representations of tasks-sets in VLPFC (one or multiple sets of S-R associations together with additional requirements and rules that govern a task) influence the lower-level processing and representation of S-R sets in SPL. The larger amount of influence to the left anterior SPL from left VLPFC in task 3 compared to task 2 , a significant RFX-level difference, support this notion, since task demands are more complex in task 3. The inferior parietal lobule (IPL) was shown in earlier investigation to be strongly involved in task switching in general (Smith et al., 2004), and more specifically in reconfiguring S-R mappings (Barber and Carter, 2005). Hence, the influence from the left IPL to the more dorsal anterior SPL could be specific to 
processing to changing and reconfiguring $S-R$ associations at switches, particularly when two complex (possibly conflicting) sets of S-R associations have to be interchanged. The increased input from inferior parietal areas during switch periods in task 4, both compared to repeat periods in task 4 and to switch periods in task 2 and 3, seems to further support this idea.

The left anterior SPL shows some interesting interactions with the striatum, modulated both by task (task 2 vs. task 3 ) and task context (switch periods vs. repeat periods). In task 2, a significant increase in the influence from the bilateral putamen can be seen in the repeat periods. In task 3 increased influence to the left-side caudate nucleus is evident during switch periods. Tentatively, this seems to reflect two-fold role attributed to the striatum in earlier studies: transformation of concrete stimulus exemplar information into motor responses (Cools et al., 2004; Crone et al., 2006) on the one hand, and overriding previous S-R association when switching between tasks (Crone et al., 2006), possibly by inhibition of task inappropriate responses and the execution of task appropriate responses (Shafritz et al., 2005), on the other. The influence of the putamen on activity of the left anterior SPL, more extensive in the repeat periods than in switch periods of task 2, similar to the left PMd, might indicate that putamen input is important when specific $S-R$ associations are made in a non-conflicting context. The influence of the head of the caudate nucleus on activity of the left anterior SPL, more extensive in the switch periods than in repeat periods of task 3 , could indicate that input from the caudate nucleus underlies processing to reconfigure S-R associations and to inhibit inappropriate responses.

A location more posterior in the left SPL was activated in the contrast of task 2 and task 4 response stimuli, without being identified in the earlier contrast of task 3 and 4 as the left anterior SPL was. Thus, a tendency towards dissociation of left superior parietal areas is shown, with posterior SPL associated more with response selection processes, whereas anterior SPL cortex seems to be involved in S-R association. It is therefore very interesting to look at the differences in the interactions with the rest of the brain of the posterior SPL compared to the anterior SPL.

RFX GCM analysis with the left posterior SPL as reference region shows it interactions to be very similar to those of the left anterior SPL. The main target areas of output from the left posterior SPL identified in the RFX GCMs were bilateral inferior temporal and occipito-temporal areas, and more superior and medial parietal areas. The main sources of input to the left posterior SPL consist of left ventrolateral prefrontal areas and anterior insula, the left inferior parietal lobule (IPL) extending into the left superior temporal gyrus (STG), and SMA/preSMA. The input from left IPL and VLPFC regions to the left posterior SPL seems even more extensive than for the anterior SPL, especially for tasks 3 and 4 . Furthermore, this input is significantly larger in the switch periods of tasks 3 and 4, where S-R associations and response selection get more complex. Hence, the posterior part of the SPL, like the anterior part seems to interact extensively with the VLPFC, involved in task-set processing and updating, and with the $\mathrm{IPL}$, involved in reconfiguring S-R associations. 


\section{Random effects Granger causality mapping}

RFX GCM was used to explore effective connectivity for given reference region without a priori assumptions about the connectivity of that region and the directions of its influences. The concept of Granger causality (Granger, 1969, 1980) was used to map the existence and direction influence over brain from temporal dependency information in the fMRI data (Goebel et al., 2003; Roebroeck et al., 2005). Alternative approaches to effective connectivity, such as Covariance Structural Equation Modeling (Buchel and Friston, 1997; Mclntosh and Gonzalez-Lima, 1994) and Dynamic Causal Modeling (Friston et al., 2003), require prior specification of involved regions and the connections between them in a directed graph model. Although this allows testing of specific hypotheses about influence along known connections between regions and its modulation by experimental factors, these approaches are less applicable when the network of regions and connections underlying a specific tasks is not well known. Here, statistical inference for Granger causality maps was extended to the random effects level such that the consistency of explored regional interactions over a group of subjects could be assessed. This technique was shown to be useful in identifying the interactions of premotor and parietal cortex in switching visuomotor association tasks, which were largely unknown to date. Some caution has to be exerted in interpreting GCMs, as discussed in Roebroeck et al. (2005). First, because relative temporal precedence in the BOLD signals of two regions is used to evaluate existence and directions of causal influence, note must be taken of the possible disturbing effect on GCM inferences of differences in the local hemodynamics over the brain. A Granger causal influence between two cortical regions could result not only from their interactions at the neural level, but also from mere differences in local physiology of capillaries, blood flow and vasodilation underlying the Blood Oxygenation Level Dependent (BOLD) signal. Therefore, it was argued (Roebroeck et al., 2005) that Granger causal influences in fMRI data should show modulation by experimental parameters manipulating cognitive context to assign inferred influences to neuronal interactions, not to differences in hemodynamics. Here, consistent influences between regions were shown over a group of 15 subjects, and, moreover, significant modulation of these influence experimental factors (switch period vs, stay period and between task modulations) was shown at the random-effects level, ruling out alternative explanations by mere hemodynamics. Second, because the GCM technique maps influences between regions considering them as a single interacting pair, no sound conclusions can be drawn about the 'directness' of these interactions, i.e. whether or not an influence could be mediated by a third region or structure. Assessing direct or indirect influences requires estimation of a multivariate model that contains all three regions, e.g. a 3-dimensional autoregressive model to estimate conditional Granger causality or a dynamic causal model. However, multivariate connectivity models containing many regions cannot be estimated before all regions involved are identified, which is exactly what GCM is intended to do. In this sense, demanding full information on direct or indirect interactions in an early exploratory phase of connectivity analysis means running a circular argument. Third, it was argued (Roebroeck et al., 2005) that, to obtain unbiased statistics for GCMs, inference must be performed on difference maps (dGCMs) of the form Ref2Vox - Vox2Ref, which assign either a influence from reference region to a map voxel (positive value), or an influence 
from a map voxel to the reference region (negative value), but not both at the same time. This means that true bidirectional interactions between regions of equal strength and time-delay are lost. Only the dominant direction of influence is made visible.

\section{Conclusion}

In a fast event-related fMRI study four switching visuomotor association tasks were compared to investigate the regions underlying the different component processes: response selection, competition and inhibition and stimulus-response association. Random effects Granger causality mapping (RFX GCM) was used to identify the consistent interaction between regions involved at the group level, and the modulation of these interactions by task-type and switching-context. The networks of regions interacting with dorsal premotor cortex and superior parietal cortex to perform switching visuomotor association tasks were identified. This gives important insight into complex brain dynamics underlying arbitrary visuomotor association tasks and task switching in general. Furthermore, RFX GCM was shown to be a useful tool in exploring interactions in unknown large-scale brain networks that underlie the performance of specific tasks. 


\section{References}

Asari T, Konishi S, Jimura K, Miyashita Y (2005) Multiple components of lateral posterior parietal activation associated with cognitive set shifting. Neuroimage 26: 694-702.

Barber AD, Carter CS (2005) Cognitive control involved in overcoming prepotent response tendencies and switching between tasks. Cereb Cortex 15: 899-912.

Boynton GM, Engel SA, Glover GH, Heeger DJ (1996) Linear systems analysis of functional magnetic resonance imaging in human V1. J Neurosci 16: 4207-4221.

Brass M, von Cramon DY (2004) Decomposing components of task preparation with functional magnetic resonance imaging. J Cogn Neurosci 16: 609-620.

Braver TS, Reynolds JR, Donaldson DI (2003) Neural mechanisms of transient and sustained cognitive control during task switching. Neuron 39: 713-726.

Buchel C, Friston KJ (1997) Modulation of connectivity in visual pathways by attention: cortical interactions evaluated with structural equation modelling and fMRI. Cereb Cortex 7: 768778.

Bunge SA (2004) How we use rules to select actions: a review of evidence from cognitive neuroscience. Cogn Affect Behav Neurosci 4: 564-579.

Bunge SA, Hazeltine E, Scanlon MD, Rosen AC, Gabrieli JD (2002) Dissociable contributions of prefrontal and parietal cortices to response selection. Neuroimage 17: 1562-1571.

Carter CS, Braver TS, Barch DM, Botvinick MM, Noll D, Cohen JD (1998) Anterior cingulate cortex, error detection, and the online monitoring of performance. Science 280: 747-749.

Cavina-Pratesi C, Valyear KF, Culham JC, Kohler S, Obhi SS, Marzi CA, Goodale MA (2006) Dissociating arbitrary stimulus-response mapping from movement planning during preparatory period: evidence from event-related functional magnetic resonance imaging. $\mathrm{J}$ Neurosci 26: 2704-2713.

Colby CL, Goldberg ME (1999) Space and attention in parietal cortex. Annu Rev Neurosci 22: 319349.

Cools R, Clark L, Robbins TW (2004) Differential responses in human striatum and prefrontal cortex to changes in object and rule relevance. J Neurosci 24: 1129-1135.

Crone EA, Wendelken C, Donohue SE, Bunge SA (2006) Neural evidence for dissociable components of task-switching. Cereb Cortex 16: 475-486.

Desimone R, Duncan J (1995) Neural mechanisms of selective visual attention. Annu Rev Neurosci 18: $193-222$.

Dove A, Pollmann S, Schubert T, Wiggins CJ, von Cramon DY (2000) Prefrontal cortex activation in task switching: an event-related fMRI study. Brain Res Cogn Brain Res 9: 103-109.

Epstein R, Kanwisher N (1998) A cortical representation of the local visual environment. Nature 392: 598-601.

Fischl B, Sereno MI, Tootell RB, Dale AM (1999) High-resolution intersubject averaging and a coordinate system for the cortical surface. Hum Brain Mapp 8: 272-284.

Friston KJ, Harrison L, Penny W (2003) Dynamic causal modelling. Neuroimage 19: 1273-1302.

Genovese CR, Lazar NA, Nichols T (2002) Thresholding of statistical maps in functional neuroimaging using the false discovery rate. Neuroimage 15: 870-878.

Goebel R, Roebroeck A, Kim DS, Formisano E (2003) Investigating directed cortical interactions in time-resolved fMRI data using vector autoregressive modeling and Granger causality mapping. Magn Reson Imaging 21: 1251-1261. 
Granger CWJ (1969) Investigating causal relations by econometric models and cross-spectral methods. Econometrica 37: 424-438.

Granger CWJ (1980) Testing for causality: A Personal Viewpoint. Journal of Economic Dynamics and Control 2: 329-352.

Kanwisher N, McDermott J, Chun MM (1997) The fusiform face area: a module in human extrastriate cortex specialized for face perception. J Neurosci 17: 4302-4311.

Koechlin E, Ody C, Kouneiher F (2003) The architecture of cognitive control in the human prefrontal cortex. Science 302: 1181-1185.

Konishi S, Hayashi T, Uchida I, Kikyo H, Takahashi E, Miyashita Y (2002) Hemispheric asymmetry in human lateral prefrontal cortex during cognitive set shifting. Proc Natl Acad Sci U S A 99: 7803-7808.

Konishi S, Nakajima K, Uchida I, Kameyama M, Nakahara K, Sekihara K, Miyashita Y (1998) Transient activation of inferior prefrontal cortex during cognitive set shifting. Nat Neurosci 1: $80-84$

Kriegeskorte N, Goebel R (2001) An efficient algorithm for topologically correct segmentation of the cortical sheet in anatomical mr volumes. Neuroimage 14: 329-346.

McIntosh AR, Gonzalez-Lima F (1994) Structural Equation Modeling and Its Application to Network Analysis in Functional Brain Imaging. Human Brain Mapping 2: 2-22.

Miller EK, Cohen JD (2001) An integrative theory of prefrontal cortex function. Annu Rev Neurosci 24: 167-202.

Monchi O, Petrides M, Petre V, Worsley K, Dagher A (2001) Wisconsin Card Sorting revisited: distinct neural circuits participating in different stages of the task identified by eventrelated functional magnetic resonance imaging. J Neurosci 21: 7733-7741.

Monsell S (2003) Task switching. Trends Cogn Sci 7: 134-140.

Roebroeck A, Formisano E, Goebel R (2005) Mapping directed influence over the brain using Granger causality and fMRI. Neuroimage 25: 230-242.

Rushworth MF, Hadland KA, Paus T, Sipila PK (2002) Role of the human medial frontal cortex in task switching: a combined fMRI and TMS study. J Neurophysiol 87: 2577-2592.

Shafritz KM, Kartheiser P, Belger A (2005) Dissociation of neural systems mediating shifts in behavioral response and cognitive set. Neuroimage 25: 600-606.

Smith AB, Taylor E, Brammer M, Rubia K (2004) Neural correlates of switching set as measured in fast, event-related functional magnetic resonance imaging. Hum Brain Mapp 21: 247-256.

Talairach J, Tournoux P (1988) Co-planar stereotaxic atlas of the human brain: 3-dimensional proportional system, an approach to cerebral imaging. Stuttgart: Thieme.

Thoenissen D, Zilles K, Toni I (2002) Differential involvement of parietal and precentral regions in movement preparation and motor intention. J Neurosci 22: 9024-9034.

Wager TD, Sylvester CY, Lacey SC, Nee DE, Franklin M, Jonides J (2005) Common and unique components of response inhibition revealed by fMRI. Neuroimage 27: 323-340.

Wise SP, Murray EA (2000) Arbitrary associations between antecedents and actions. Trends Neurosci 23: 271-276.

Wylie GR, Javitt DC, Foxe JJ (2006) Jumping the Gun: Is Effective Preparation Contingent upon Anticipatory Activation in Task-relevant Neural Circuitry? Cereb Cortex 16: 394-404.

Yeung N, Nystrom LE, Aronson JA, Cohen JD (2006) Between-task competition and cognitive control in task switching. J Neurosci 26: 1429-1438. 


\title{
High resolution diffusion tensor imaging and tractography of the human optic chiasm at 9.4T
}

\begin{abstract}
The optic chiasm with its complex fiber micro-structure is a challenge for diffusion tensor models and tractography methods. At the same time, because the macroscopic connectivity of the optic chiasm is well known it is an ideal candidate for validation of Diffusion Tensor Imaging tractography approaches in resolving inter-regional

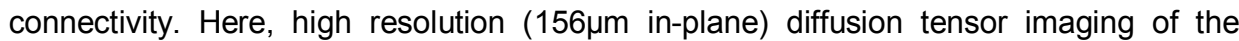
human optic chiasm was performed ex vivo at ultra-high field (9.4T). Estimated diffusion tensors at this high resolution were able to capture complex fiber configurations such as sharp curves, and convergence and divergence of tracts, but were unable to resolve directions at sites of crossing fibers. Despite the complex microstructure of the fiber paths through the optic chiasm, all known connections could be tracked by a line propagation algorithm, although fibers crossing from the optic nerve to contralateral tract were heavily underrepresented, whereas ipsilateral nerve-to-tract connections, as well as tract-to-tract connections were overrepresented and erroneous nerve-to-nerve connections were tracked. The effects of spatial resolution and the varying degrees of partial volume averaging of complex fiber architecture on the performance of these methods could be investigated. Errors made by the tractography algorithm at high resolution were shown to increase at lower resolutions closer to those used in vivo. This study shows that post-mortem investigation of fixed tissue samples with DTI at high field strengths is important in the validation of MR-based diffusion models and tractography algorithms.
\end{abstract}

Based on: Roebroeck A, Chiry O, Formisano E, Galuske R, Goebel R. High resolution diffusion tensor imaging and tractography of the human optic chiasm at 9.4T. In preparation. 


\section{Introduction}

Diffusion tensor imaging (DTI) is a magnetic resonance imaging technique that can quantify the directional dependence of water diffusion in biological tissue. In brain whitematter tissue, water diffusion is greater along the direction of axon-bundles than perpendicular to it. By quantifying the degree and direction of this diffusion anisotropy, DTI can be used to probe the micro-structure of white-matter fiber-bundles noninvasively in vivo. Fiber tracking or tractography refers to the derivation of area-to-area axonal connectivity from the properties of local diffusion profiles. In its simplest form, tractography amounts to following the local primary axis of diffusion to proceed from one area to another along the fiber-structures anatomically connecting the two. Many variants of this line propagation techniques have been proposed (Basser et al., 2000; Conturo et al., 1999; Jones et al., 1999; Lazar et al., 2003; Lori et al., 2002; Mori et al., 1999; Poupon et al., 2000; Westin et al., 2002; Xue et al., 1999), and many of the large white matter structures such as the cortical association tracts can be reliably traced in individual subjects using these methods (Catani et al., 2002; Mori et al., 2002; Wakana

et al., 2004). However, DTI tractography is known to have limitations primarily resulting from partial-volume averaging of complex fiber-architecture (such as sharp curves and crossings) into single voxels (Alexander et al., 2001; Tuch et al., 2002; Tuch et al., 2003). In this study high resolution (156 $\mu \mathrm{m}$ in-plane) diffusion tensor imaging of the human optic chiasm was performed ex vivo at ultra-high field (9.4T). Water diffusion anisotropy has been shown to be preserved in formaline-fixed tissue (Guilfoyle et al., 2003; Sun et al., 2005; Sun et al., 2003). This makes the post-mortem investigation of fixed tissue samples with DTI at high field strengths an attractive candidate for the validation of the diffusion tensor model and tractography algorithms and the effects of spatial resolution on their performance. The optic chiasm, with its complex microstructure of fibers, which kiss, cross, converge and curve, is a challenge for the capabilities of the diffusion tensor model and tractography methods. Since the macroscopic connectivity through the optic chiasm is well known it is an ideal candidate for validation of DTI tractography approaches in resolving inter-regional connectivity.

\section{Diffusion tensor imaging}

Within each voxel, the local diffusion profile is captured in a 3-dimensional rank-2 tensor: the diffusion tensor (Basser et al., 1994) that can be geometrically represented as an ellipsoid. The diffusion tensor (DT) models the diffusion profile in a voxel as a 3-D gaussian with three orthogonal eigenvectors or axes of diffusion (the primary or major eigenvector $\mathbf{e}_{1}$, the secondary or median eigenvector $\mathbf{e}_{2}$, and the tertiary or minor eigenvector $\mathbf{e}_{3}$ ) and their corresponding eigenvalues or diffusion constants $\lambda_{1}, \lambda_{2}$, and $\lambda_{3}$. DTI is enabled by diffusion-weighted MR sequences that are sensitized (weighted) at each acquisition for diffusion in a particular direction. The diffusion tensor models the signal $S$ for a diffusion-weighting direction $\mathbf{g}=\left(g_{x}, g_{y}, g_{z}\right)$ with respect to a non diffusion weighted image $S_{0}$ as:

$$
S / S_{0}=e^{b \mathbf{g}^{\mathrm{T}} \mathbf{D g}}
$$


Where $b$ is the b-value, quantifying the strength of diffusion weighting. The six parameters of the DT can be estimated at each voxel if at least six measurements weighted for diffusion along six non-collinear directions are acquired, along with an unweighted acquisition. Useful scalar quantities can be derived from the eigenvalues $\lambda_{1} \lambda_{2}$, and $\lambda_{3}$ of the estimated diffusion tensors that correspond to its primary, secondary and tertiary axes of diffusion, respectively. Such scalar quantities classify the tensor shape and are independent of the direction of the diffusion axes (i.e. they will be invariant under rotations of the tensor). The mean diffusivity is given by:

$$
M D=\frac{\operatorname{Trace}(D)}{3}=\frac{\lambda_{1}+\lambda_{2}+\lambda_{3}}{3}
$$

The mean diffusivity coefficient is proportional to the volume of the ellipsoid and quantifies the absolute amount of diffusion in a voxel. It will be large where unrestricted diffusion takes place, such as in free diffusing fluids, and smaller where diffusion is restricted, such as in biological tissue. However, the real interest in DTI lies with anisotropy, indicative of the restriction of diffusion along specific directions such as occurs in brain white matter. Anisotropy measures essentially quantify the amount of variance present in the three eigenvalues of the diffusion tensor. One of the most used anisotropy measures is the Fractional Anisotropy (Pierpaoli and Basser, 1996), given by:

$$
F A=\frac{\sqrt{3\left(\lambda_{1}-\bar{\lambda}\right)^{2}+3\left(\lambda_{2}-\bar{\lambda}\right)^{2}+3\left(\lambda_{3}-\bar{\lambda}\right)^{2}}}{\sqrt{2\left(\lambda_{1}^{2}+\lambda_{2}^{2}+\lambda_{3}^{2}\right)}}, \quad \bar{\lambda}=\frac{\lambda_{1}^{2}+\lambda_{2}^{2}+\lambda_{3}^{2}}{3}
$$

Fractional anisotropy is high where tensors have a highly linear or prolate shape (similar to a cigar), with the primary eigenvalue much larger than the other two, indicating the presence of a single highly coherent fiber bundle. With more oblate (planar, pancakelike) or spherical shapes, FA will decrease. Although FA is highly suitable to quantify the absolute amount of anisotropy (effectively the deviation from a spherical shape), it is not able to distinguish well between linear and planar tensor shapes. Geometric measures of diffusion have been proposed that are better suited to discern linear from planar and spherical tensor shapes (Westin et al., 2002):

$$
C_{l}=\frac{\lambda_{1}-\lambda_{2}}{\lambda_{1}}, C_{p}=\frac{\lambda_{2}-\lambda_{3}}{\lambda_{1}}, C_{s}=\frac{\lambda_{3}}{\lambda_{1}}, C_{l}+C_{p}+C_{s}=1
$$

The linear measure $C_{l}$ is high for linear or prolate tensor shapes, where $\lambda_{1} \gg \lambda_{2} \approx \lambda_{3}$. The planar measure $C_{p}$ is high for planar or oblate tensor shapes, where $\lambda_{1} \approx \lambda_{2}>>\lambda_{3}$. The spherical measure $C_{s}$ is high for spherical tensor shapes, where $\lambda_{1} \approx \lambda_{2} \approx \lambda_{3}$. A quantity similar in behavior to FA is $1-C_{s}$, quantifying the 
deviation from a spherical shape, without distinguishing between linear and planar shapes. A quantity which is better able to identify non-linear tensor shapes, is $1-C_{l}$, quantifying the deviation from a linear shape.

The diffusion tensor model has its limitation in representing the local diffusion profile within a voxel. Since typical voxel sizes for in-vivo human DTI are in the range of $2 \mathrm{~mm}$ (i.e. $8 \mathrm{~mm}^{3}$ ), some white-matter voxels can easily contain fiber bundles with different directions. The chief limitations of the diffusion tensor is in representing two fiber-bundles with different directions that cross within a voxel, which gives rise to an averaged diffusion profile with two maxima. The diffusion tensor model is a 3-D Gaussian profile constrained to a single maximum. When two fiber-bundles cross at (nearly) ninety degrees, the tensor will take an oblate (pancake-like) shape in the plane of crossing. When bundles cross at less than ninety degrees the tensor will have a prolate shape with its primary axes in between those of the directions of the crossing bundles. In either case, the estimated tensor model misrepresents the actual diffusion profile. Adding to the problem, the DT will also be unable to distinguish a crossing $(X)$ from a kissing $(><)$ configuration, since both give rise to the same actual diffusion profile. Another situation that cannot be accurately represented by the DT is when a single tract curves sharply within a single voxel. This would lead to a continuous range of directions present in a single voxel, a non-gaussian diffusion profile which the DT is unable to represent since it assumes the presence of a single coherent direction. An estimated diffusion tensor model for such a voxel will quickly degrade towards an oblate or spherical shape which only represents the average direction of the curve at a low modeled anisotropy.

Fiber tracking

Constructing a continuous tract-line $r(s)=(x(s), y(s), z(s))$ from a tensor field $\mathbf{D}(x, y, z)$, can be formulated as the solution of an ordinary differential equation (Basser et al., 2000):

$$
\frac{d \mathbf{r}(s)}{d s}=f(\mathbf{D}(\mathbf{r}(s)))
$$

Where $r(s)$ is parameterized by position s along the tract, $\boldsymbol{D}(\boldsymbol{r}(s))$ denotes the tensor at position $r(s)$ for a given $s$, and $f$ denotes a function giving the tangent to $r(s)$ from the local tensor at $s$. In practice a discrete tract-line $r(i)=\left(x_{i}, y_{i}, z_{i}\right)$, with $i=0, \ldots, \mathrm{N}$, will have to be constructed from a discrete estimated tensor volume $D(i, j, k)$. Line propagation or streamline approaches to fiber tracking trace a tract-line through a tensor volume by following the local estimated diffusion directions from a seedpoint. The seedpoint $\left(x_{0}, y_{0}\right.$, $z_{0}$ ) forms the boundary condition for the numerical process of solving a discretized differential equation. In its simplest form a line propagation algorithm amounts to approximating a solution by Euler's method:

$$
\mathbf{r}(i+1)=\mathbf{r}(i)+\alpha * \mathbf{v}(i), \quad \mathbf{v}(i)=f(\mathbf{D}(\mathbf{r}(i)), \mathbf{v}(i-1))
$$


Starting from the seedpoint a step of stepsize $\alpha$ is taken in a direction $\mathbf{v}(i)$ determined by the local tensor $\mathbf{D}$ and the previous tracking direction $\mathbf{v}(i-1)$. The simplest form of direction determination would be to set $\mathbf{v}(I)=\mathbf{e}_{1}(\mathbf{r}(I))$, i.e. to use the primary eigenvector of the local tensor as the new tracking direction. The Euler method as an ODE solver is known to be unstable and inaccurate when stepsizes are not extremely small. Better methods exist that use information at multiple points near the step-location to yield better stability and accuracy, such as the fourth order Runge-Kutta method (Press et al., 2002). Taking the primary eigenvector of the local tensor as the new tracking direction can work well in regions where a single coherent tract is present. However, this strategy can lead to errors when more complex configurations, such as crossings, are encountered. As discussed above, the primary eigenvector often does not represent a veridical tract direction in the case of a crossing. An approach to direction determination that uses the full local tensor information can perform better in this case. It has been proposed to use the local tensor $D$ as a projection operator to deflect the previous tracking direction towards the directions compatible with the local tensor(Lazar et al., 2003; Westin et al., 2002), by defining $f$ as:

$$
\mathbf{v}(i)=f(\mathbf{D}(\mathbf{r}(i)), \mathbf{v}(i-1))=\mathbf{D}(\mathbf{r}(i)) * \mathbf{v}(i-1)
$$

The new tracking direction is formed as the matrix-vector product of the local tensor and the old tracking direction. In cases of fiber-crossing, the inertia of the old direction will lead this operation to trace through the planar tensor at the crossing site, when using only the primary eigenvector might fail. It should be noted that this inertial bias might also lead to a wrong interpretation of kissing sites or high curvature bends as crossings where a tract continues a relatively straight path. To avoid noise contributions from eigenvectors with small eigenvalues, only the larger eigenvectors can be made to contribute to the projection operation. The full tensor can be written in terms of its eigenvector and eigenvalues:

$$
\mathbf{D}=\lambda_{1} \mathbf{e}_{1} \mathbf{e}_{1}^{T}+\lambda_{2} \mathbf{e}_{2} \mathbf{e}_{2}^{T}+\lambda_{3} \mathbf{e}_{3} \mathbf{e}_{3}^{T}
$$

This sum can be truncated to include only the terms that are considered to be large enough. A possible truncation strategy is that of Westin et al. (Westin et al., 2002), who propose to select the contributing eigenvectors from the relative magnitudes of the $C_{l}$, $C_{p}$, and $C_{s}$ coefficients. When $C_{l}$ is largest, only $\mathbf{e}_{1}$ is used, when $C_{p}$ is largest $\mathbf{e}_{1}$ and $\mathbf{e}_{2}$ are used, and the full tensor is used when $C_{s}$ is largest.

Some inherent limitations exist in deriving with certainty the existence or absence of anatomical connections between brain regions from tractography results. The problem of directional polarity and the limitations of the DT model in representing highly curving or crossing fibers have already been mentioned. Line propagation algorithms will often stop at crossings or high curvature regions, either because the estimated anisotropy does not exceed a fixed threshold, or because of the often inbuilt bias against high curvature tracts in the form of the angle threshold. Additionally, situations of convergence (multiple bundles merge into one) and/or divergence (a bundle splits into two) also yield considerable trouble for DT tractography approaches. At a voxel where convergence 
takes place, information is lost about which fibers merged into the main tract and which were present already. Similarly, at the site of divergence it is unclear which axon-bundles leave the main tract and which continue along it. If converging and diverging bundles maintain a coherent location within the main tract as they follow it along, an increase in spatial resolution may resolve some of the ambiguity. The idea is that if the main tract is sampled densely enough, the diverging or converging parts might be kept separate as the tract continues its course. Problems with high curvature tracts can also be mitigated by an increase in spatial resolution. If voxel size can be reduced beyond the radius of tract curvature, the locally estimated tensor models will become sharper defined. Potentially, increased spatial resolution can resolve some kissing vs. crossing situations in a similar way. If the curvature of the tracts at the kissing point is low enough with respect to the voxel size and the 'kiss' is not too close, each might be followed through without erroneously crossing over.

\section{The anatomy of the human optic pathways and optic chiasm}

The optic nerves, arising from the ganglion cells of the retina, project in largest part to the lateral geniculate nucleus (LGN) in the thalamus. From the LGN, the optic radiation projects to the primary visual cortex in the calcarine fissure of the occipital lobe (see figure 1). A retinotopic organization is kept throughout the projections from the eye to the occipital cortex. The optic nerves pass dorsally into the brain, ventral to the orbitofrontal cortex, to meet at the midline in the optic chiasm, an oblate structure where the optic fibers partially decussate and merge into the optic tracts. Optic fibers carrying input from the temporal half of the retina (including the temporal half of the fovea), representing the contralateral half of the visual field pass into the ipsilateral optic tract in the optic chiasm. Fibers arising from the nasal hemiretinae, representing the ipsilateral visual hemifield, decussate in the optic chiasm to enter the contralateral optic tract. Decussating fibers do not cross the chiasm in an exclusively diagonally oriented path. Rather, they curve sharply (even looping backwards) to cross the chiasm in a medio-lateral direction, then curve sharply again to align with the temporal fibers of contralateral optic nerve and enter into the contralateral optic tract (Standring, 2005). Such highly curved paths are a problem for most fiber tracking algorithms that implicitly assume fibers to be guided by shortest path and minimum curvature constraints. 


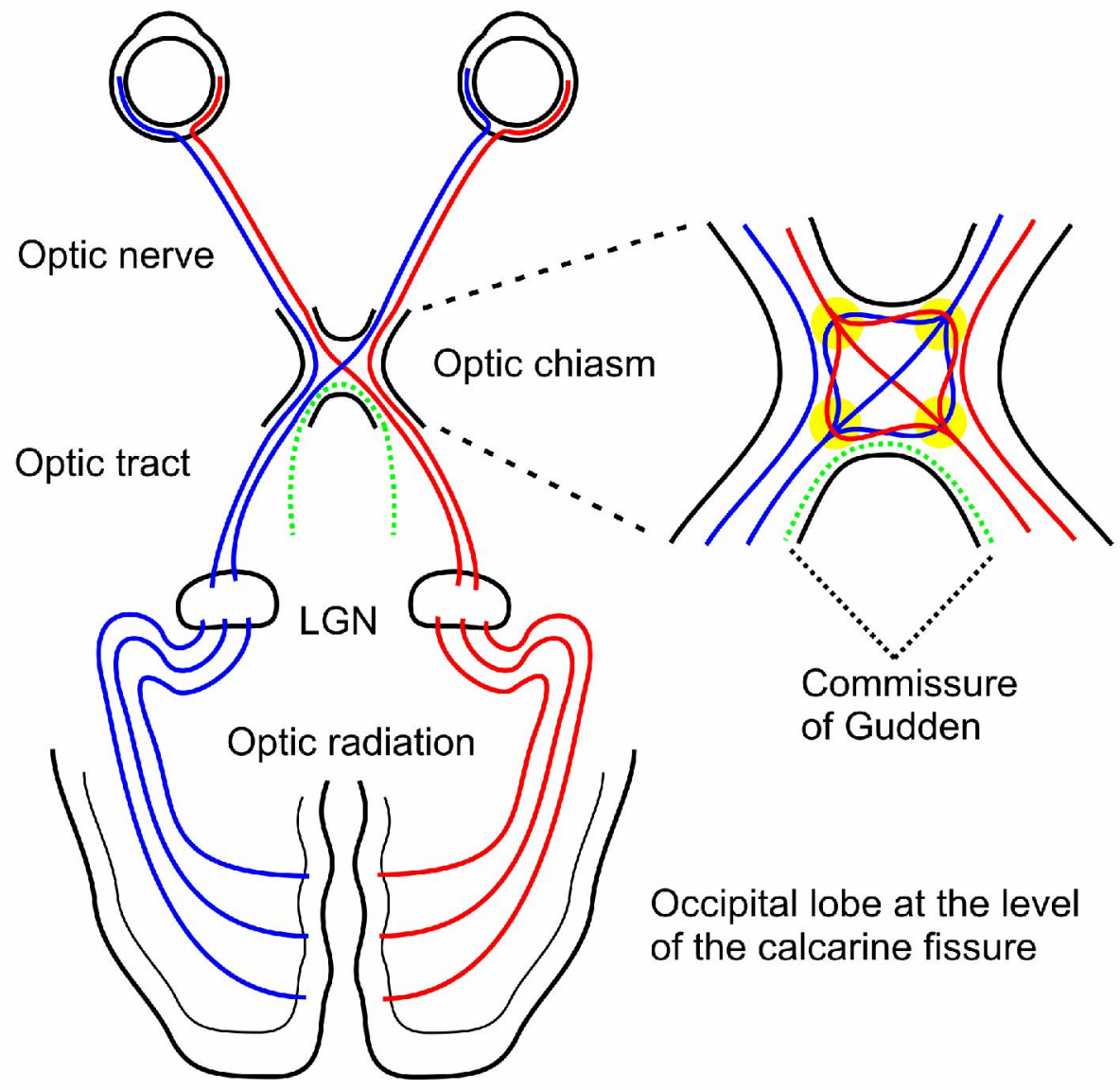

Figure 1: The anatomy of the human optic pathways and optic chiasm. Red Lines are nerves representing the left visual hemi-field, blue lines are nerves representing the right visual hemi-field. Yellow circles mark regions of high curvature, crossings, divergence and convergence, problematic for the diffusion tensor model and line propagation algorithms. LGN: Lateral Geniculate Nucleus.

The majority of all optic nerve fibers cross in the chiasm and about two thirds of the central chiasm is occupied by fibers arising from the foveal region of the retinae, passing mostly through the dorsal part of the central chiasm. The fibers arising from the extrafoveal part of the nasal hemi-retinae decussate more dorsally in the optic chiasm. The ventral-most portion of the central chiasm is crossed by fibers representing the lateral monocular part of the visual hemifields (Standring, 2005). The posterior and medial part of the optic chiasm is thought to contain fibers that are not derived from the optic nerves. These fibers form the ventral supra-optic commissure or commissure of 
Gudden, possibly connecting the medial geniculate nuclei of the two sides. Gudden's commisure is one of the three supra-optic commisures, which also include the dorsal supra-optic commissure (commissure of Meynert), and the anterior supra-optic commissure (commissure of Ganser). The connections of the supra-optic commissures in humans are uncertain, and only the dorsal and ventral commissures are recognized in most anatomical nomenclature.

\section{Methods}

\section{Tissue preparation}

We examined brains of four subjects (two female, 23 and 33 years old at death; two male, 26 and 34 years old at death) free of known neurological disorders. After a postmortem delay of 8 to 15 hours, the optical chiasm along with proximal aspects of the optic nerves and optic tracts (and in two cases parts of the hypothalamus) were prepared and fixated for 48 hours in a solution containing $2.6 \%$ paraformaldehyde (PA), $0.8 \%$ iodacetic acid, $0.8 \%$ sodiumperiodate, and $0.1 \mathrm{M} \mathrm{D-L-lysine} \mathrm{in} 0.1 \mathrm{M}$ phosphate buffer at $\mathrm{pH}$ 7.4. Subsequently, the tissue was washed in phosphate buffer and stored in $2 \%$ PA in phosphate buffer at $\mathrm{pH} 7.4$ in the dark at $37^{\circ} \mathrm{C}$. The time between fixation and MR scanning of the tissue ranged from 2 to 6 weeks.

\section{MR Imaging}

Experiments were performed with a $9.4 \mathrm{~T} / 31 \mathrm{~cm}$ horizontal MRI scanner (Magnex Scientific, UK) equipped with $30 \mathrm{G} / \mathrm{cm}$ gradients $(11 \mathrm{~cm} \mathrm{ID,} 300 \mu \mathrm{s}$ rise time, Magnex Scientific) and driven by a Unity INOVA console (Varian, Walnut Creek, CA). The RF coil was a home-built quadrature surface coil, composed of two $3.3 \mathrm{~cm}$ diameter partially overlapping coils. Diffusion weighted MR-images were acquired using a multi-shot pseudo-3D double spin-echo echo-planar imaging (SE-EPI) sequence with two phase encoding directions and four segments per pseudo-slice. The following parameters were used: $\mathrm{TR} / \mathrm{TE}=2000 \mathrm{~ms} / 35 \mathrm{~ms},|\mathrm{~g}|=17$ gauss $/ \mathrm{cm}, \delta=6 \mathrm{~ms}, \Delta=15 \mathrm{~ms}\left(\mathrm{~b}=1584 \mathrm{~s} / \mathrm{mm}^{2}\right.$ for each direction). Six gradient combinations according to the direction-scheme $(X, Y, 0)$, $(X, 0, Z),(0, Y, Z),(X,-Y, 0),(0, Y,-Z),(-X, 0, Z)$ and one image with minimal gradient strength on three directions was acquired as the un-weighted image. The field of view (FOV) was $2 \times 2 \times 2 \mathrm{~cm}^{3}$, the matrix size was $128 \times 128 \times 64$ (giving a nominal resolution of $156 \times 156 \mu \mathrm{m}$ in-plane with a slice thickness of $312 \mu \mathrm{m}$ ). A total of 64 repetitions for each direction were acquired and averaged. No spatial transformations were applied to the imaging volumes. One dataset had to be discarded due to inferior data quality. Estimation of diffusion tensors and tractography was performed on the remaining three optic chiasm datasets (OC1, OC2, and OC3). 
All processing and visualization was performed in custom written C++ software, using the OpenGL graphics library (www.opengl.org) for 3-D visualization. Taking the natural logarithm on both sides of (1) and writing out the Matrix-vector multiplications in components gives:

$$
\ln (S)=\ln \left(S_{0}\right)-b g_{x}^{2} D_{x x}-b g_{y}^{2} D_{y y}-b g_{z}^{2} D_{z z}-2 b g_{x} g_{y} D_{x y}-2 b g_{x} g_{z} D_{x z}-2 b g_{y} g_{z} D_{y z}
$$

This specifies the six unique components $D_{i i}$ of the diffusion tensor in terms of the measurement parameters $\left(b\right.$ and $\mathbf{g}=\left(g_{x}, g_{y}, g_{z}\right)$ ) and the measured signals ( $S$ for the measurement weighted for diffusion in direction $\mathbf{g}$, and $S_{0}$ for the un-weighted measurement). The set of acquired diffusion gradient directions defines a linear system of equations, solvable for the tensor components $D_{i i}$ (Basser et al., 1994). This system of equations was solved in the least-squares sense by a singular value decomposition (Press et al., 2002) to estimate the diffusion tensor model voxel-wise. Each tensor was then diagonalized by Jacobi rotations (Press et al., 2002), to obtain the three eigenvectors and corresponding eigenvalues. Eigenvectors were sorted with respect to the magnitude of the corresponding eigenvalues to get the primary, secondary, and tertiary axes of diffusion $\mathbf{e}_{1}, \mathbf{e}_{2}$, and $\mathbf{e}_{3}$, and their corresponding diffusion constants $\lambda_{1} \lambda_{2}$, and $\lambda_{3}$, where $\lambda_{1}>\lambda_{2}>\lambda_{3}$. Estimated tensors are visualized as cylinders in the direction of the primary axis of diffusion $\mathbf{e}_{1}$ with length proportional to $\lambda_{1}$, and as 'boxoids', rectangular 3-Dimensional boxes with axes proportional to $\lambda_{1} \mathbf{e}_{1}, \lambda_{2} \mathbf{e}_{2}$, and $\lambda_{3} \mathbf{e}_{3}$. Both cylinders and boxoids are color coded for direction of the primary axis of diffusion via the standard directional coding scheme with left to right directions colored red, anterior to posterior directions colored green, and inferior to superior directions colored blue (Pajevic and Pierpaoli, 1999). Maps of the $C_{l}$ coefficient where used to highlight problematic regions of crossing, kissing, convergence and high curvature, with the expectancy that these regions will show a reduction in the estimated $C_{\text {/ }}$ coefficient. Fiber tracking was perfomed by a line propagation algorithm using the truncated tensor projection operator described above for local direction determination. Fourth order Runge-Kutta (Press et al., 2002) method was used to advance the tract-line at every step, with a stepsize of $50 \mu \mathrm{m}$. The local tensor $\mathbf{D}$ at an arbitrary position $\mathbf{r}(s)$ in the discrete volume $\mathbf{D}(i, j, k)$ was computed by tri-linear interpolation of the individual tensorelements. The resulting interpolated tensor was then re-diagonalized by Jacobi rotations. Tracing was stopped either when the local FA was below 0.15 , or when the angle between previous and current tracking direction was larger than 120 degrees. The very liberal angle threshold was used because high curvature bends were to be expected from the known anatomy of the optic chiasm. Volumes of interest (VOIs) where drawn as contiguous pathes of voxels on coronal slices through the left and right optic nerves and tracts of each chiasm. Seedpoints for the tractography were placed in a $3 \times 3 \times 3$ grid within each seed-voxel in a VOI. Line propagation tractography was initiated for each seedpoint, both in the positive and negative direction of the local primary eigenvector. Tracked fibers from the resulting set for each VOI were then post-selected for inclusion in each of the other three VOIs, to obtain fiber-sets with given VOls as initiation and 
termination sites. Effectively this amounts to and 'AND' operation for inclusion in both VOls (Wakana et al., 2004).

\section{Spatial down-sampling}

To investigate the effects of spatial resolution on the characterization of diffusion and tractography results, down-sampled versions of OC3 were created. The down-sampled versions were produced by averaging contiguous blocks of voxels in the original dataset into larger voxels. Three down-sampled versions of the OC3 dataset were created, averaging blocks of $1 \times 2 \times 2,2 \times 4 \times 4$, and $4 \times 8 \times 8$ voxels, respectively, where the first dimension is the slice dimension. This resulted in datasets with isotropic voxelresolutions of $312.5 \mu \mathrm{m}, 625 \mu \mathrm{m}$, and $1250 \mu \mathrm{m}$, respectively. VOls were drawn, line propagation tracking was initiated, and tracked streamlines were post-selected in each of these datasets as in the original dataset. The seedpoint grid for tractography in each voxel in the downsampled datasets was increased in density to $3 \times 4 \times 4,3 \times 5 \times 5$, and $4 \times 8 \times 8$, respectively, to obtain a similar seedpoint density to the original trackings. Resampling to the original resolution of selected parts of the down-sampled data, to illustrate partial-volume averages was performed by tri-linear interpolation of the individual tensor-elements with re-diagonalization by Jacobi rotations.

Figure 2 (next page): A, B, \& C: Near-axial slice planes through OC1 at different levels. Color coding in the slices represents the linear shape measure from $C_{1}=0$ (red) to $C_{1}=0.8$ (yellow). Overlaid on the slices are the local axes of largest diffusion, rendered as cylinders and color coded for direction (see inset). D: A 3-D rendering of the zones of low $\mathrm{C}_{\mathrm{l}}$, indicating presence of non-prolate tensor shapes due to curvature, crossing, convergence and/or divergence of tracts. 

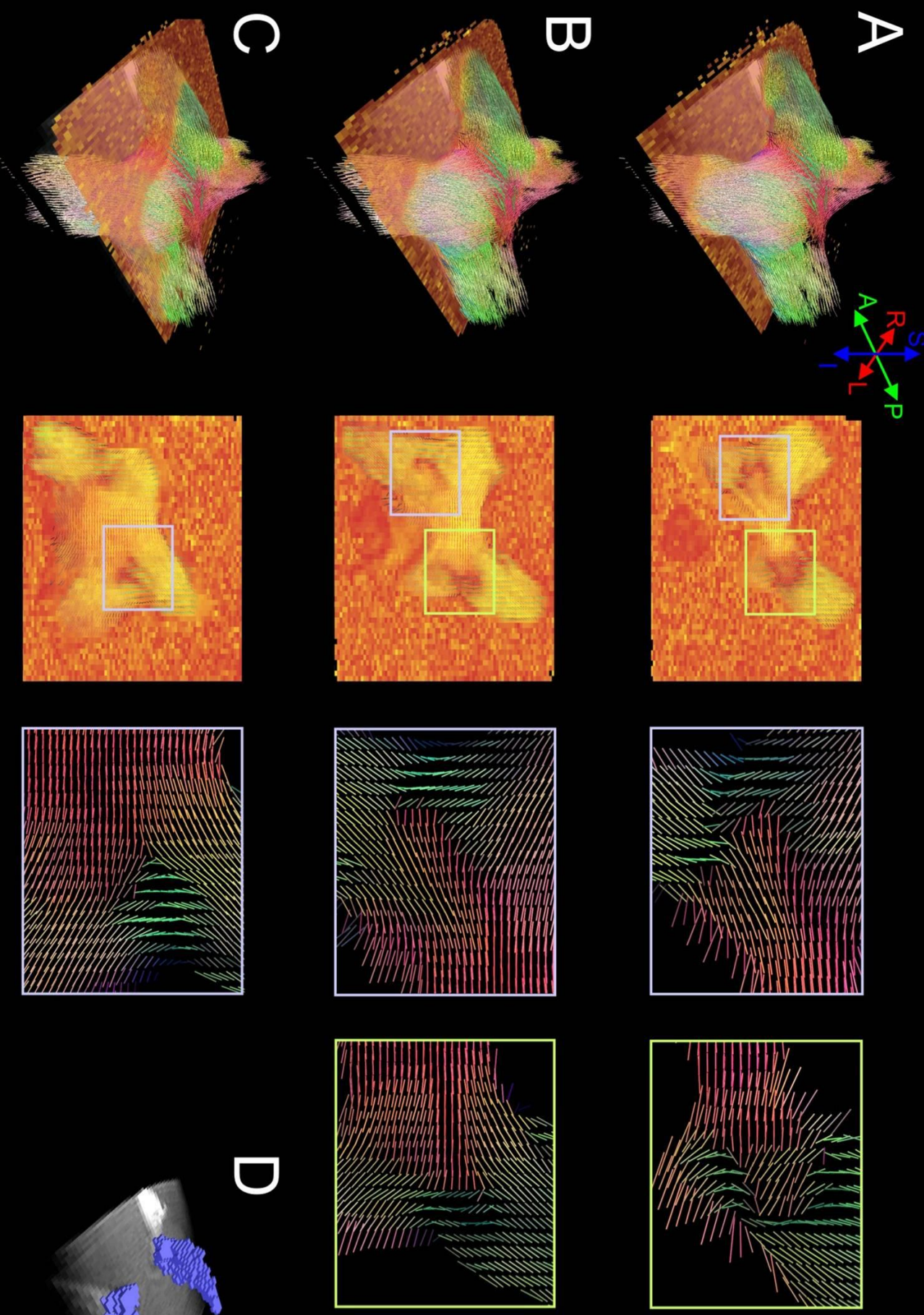


\section{Results}

Figures 2, 3 and 4 show the computed $C_{l}$ coeffient in near-axial slices for all three optic chiasm datasets: OC1, OC2, and OC3, respectively. As argued above, regions of low $C_{l}$ should be characterized by non-prolate estimated tensor shapes, indicative of curvature, crossing, and/or convergence. Each of the chiasms indeed shows clear zones of highly reduced $C_{l}$, at the lateral-most aspects of the central chiasm where it intersects with the medial aspects of the optic nerves and tracts. The enlarged detail-renderings show the estimated primary directions of diffusion to change rapidly over a very short distance in these zones, and the boxoid renderings indicate predominantly planar tensor shapes there. Visual inspection of primary eigenvector directions suggests evidence of sharp curves (e.g. Figure 2B, fourth panel; 4A, third panel), crossings (Figure 3C, third panel; Figure $4 \mathrm{~B}$, third panel) and convergence and divergence (e.g. Figure $2 \mathrm{C}$, third panel), although the interpretation is sometimes uncertain. Figures $2 D, 3 D$ and $4 D$ show a 3-D rendering of the zones of low $C_{l}$ for each of the chiasms. Interestingly, the pattern is not one of four discrete spots of high curvature and crossing (or 'pillars' in 3-D), as suggested by the yellow highlights in Figure 1, but two zones that proceed bilaterally from the anterior and ventral aspect to the posterior and dorsal aspect of the lateral central chiasm, forming diagonal bands.

Figure 3 (next page): A, B, \& C: Near-axial slice planes through OC2 at different levels. Color coding in the slices represents the linear shape measure from $C_{1}=0$ (red) to $C_{1}=0.8$ (yellow). Overlaid on the slices are the local axes of largest diffusion, rendered as cylinders, and boxoid renderings, representing all three axes of the local diffusion tensor, both color coded for direction of the primary axes of diffusion (see inset). D: A 3-D rendering of the zones of low $C_{l}$, indicating presence of nonprolate tensor shapes due to curvature, crossing, convergence and/or divergence of tracts. 

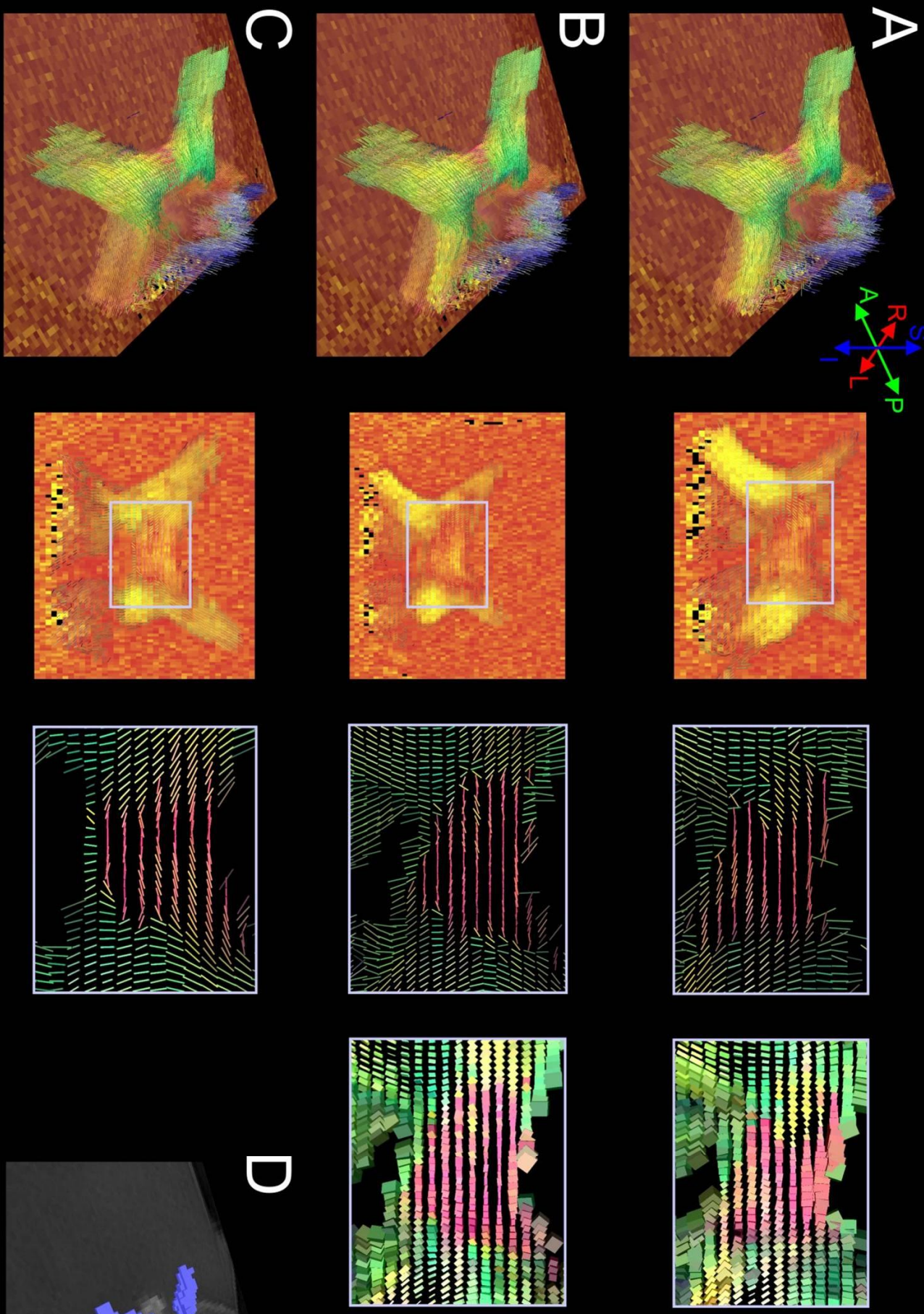
The estimated tensors for the crossing fibers in the central chiasm (see Figure 3C, third panel; Figure 4B, third \& fourth panel, but also Figures 6-8) are of particular interest. Fibers from the medial aspect of the optic nerves each pass over to the other side of midline there, and cross each other in the process. The estimated primary directions of diffusion suggest that this crossing is mostly one of a very low angle, caused by the nerves curving into a near left-right direction before passing the midline. However, the pure left-right orientation of primary eigenvector at the midline is likely to be misleading. As discussed above, the tensor model is not able to accurately represent the nongaussian multi-peaked diffusion profile of a fiber-crossing. Most likely the average direction of the two crossing fibers is what is represented at the crossing sites in the central chiasms.

Figure 4 (next page): A, B, \& C: Near-axial slice planes through OC3 at different levels. Color coding in the slices represents the linear shape measure from $C_{1}=0$ (red) to $C_{1}=0.8$ (yellow). Overlaid on the slices are the local axes of largest diffusion, rendered as cylinders, and boxoid renderings, representing all three axes of the local diffusion tensor, both color coded for direction of the primary axes of diffusion (see inset). D: A 3-D rendering of the zones of low $\mathrm{C}_{1}$, indicating presence of nonprolate tensor shapes due to curvature, crossing, convergence and/or divergence of tracts. 

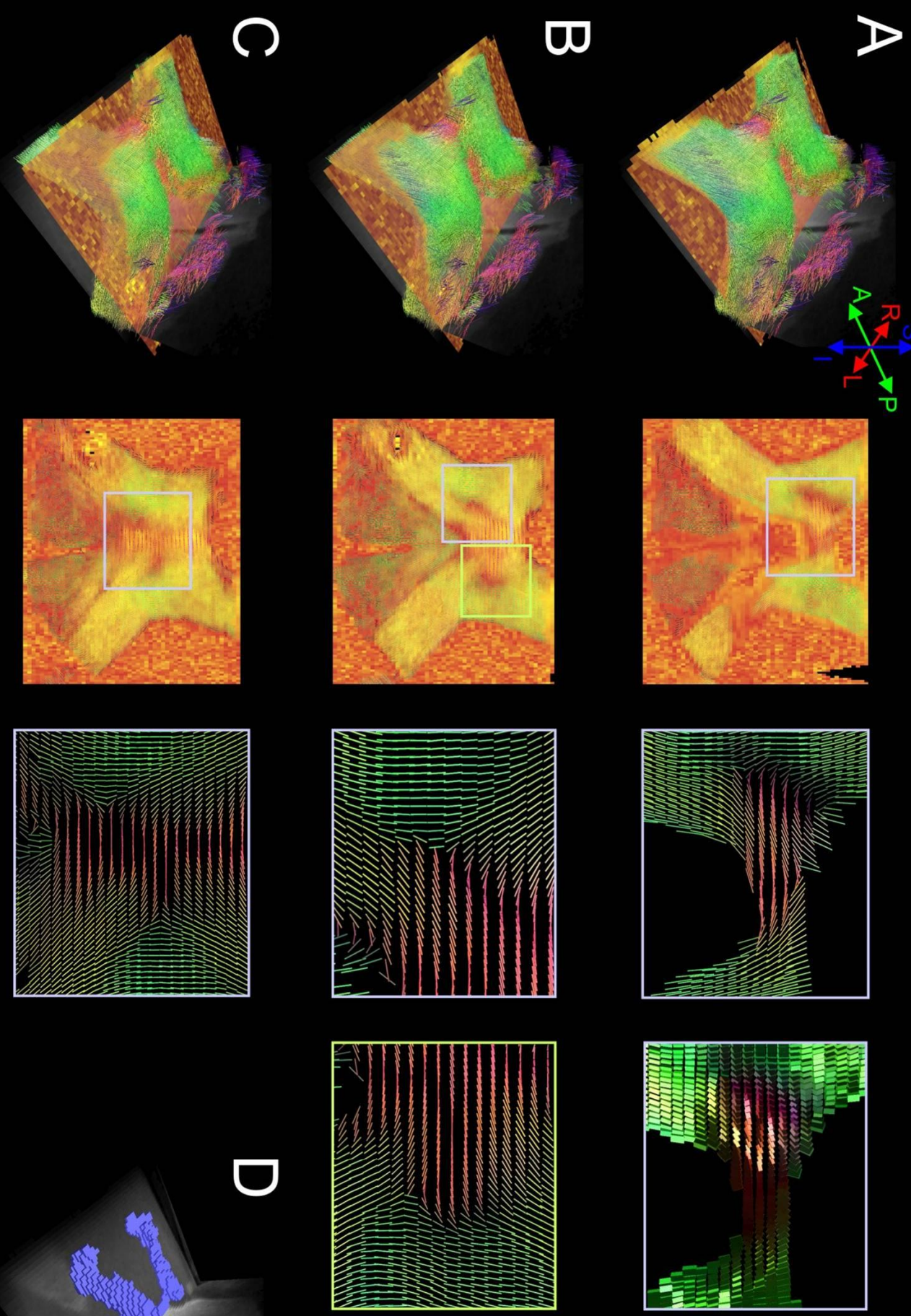
Selected streamlines resulting from the fiber trackings are shown in Figure 5. Absolute numbers and relative percentages of tracked fibers with a given seeding $\mathrm{VOI}$ and termination area are listed in Table 1. From the known anatomy of the chiasm, the majority of fibers tracked from the seed VOls in the left and right optic nerves are expected to pass through the contralateral optic tract (about 75\%). The remaining minority of streamlines should pass through the ipsilateral tract. There are no axonal connections between the eyes through the optic chiasm, so fibers should not be tracked between the VOIs in the optic nerves. Nearly the same expectations hold (mutatis mutandis) for fibers tracked from the VOls in the optic tracts. The majority should pass through the contralateral optic nerve, and a minority through the ipsilateral nerve. Connections between the two optic tracts are known to exist (see Figure 1), although these are expected to be even less numerous.

\begin{tabular}{|c|c|c|c|c|c|}
\hline \multicolumn{6}{|l|}{ OC1 } \\
\hline & & Left Nerve & Right Nerve & Left Tract & Right Tract \\
\hline Left Nerve & $(6752)$ & - & $2553(50 \%)$ & $1268(25 \%)$ & $1239(25 \%)$ \\
\hline Right Nerve & (3159) & $1222(58 \%)$ & - & $6 \quad(0.3 \%)$ & $893 \quad(42 \%)$ \\
\hline Left Tract & (2864) & $488 \quad(27 \%)$ & $0 \quad(0 \%)$ & - & $1322(73 \%)$ \\
\hline Right Tract & (7252) & $1384(32 \%)$ & 812 (19\%) & $2121(49 \%)$ & - \\
\hline
\end{tabular}

OC2

Left Nerve (3198)

\begin{tabular}{lllllll} 
Left Nerve & \multicolumn{2}{l}{ Right Nerve } & \multicolumn{2}{l}{ Left Tract } & \multicolumn{2}{l}{ Right Tract } \\
\hline- & 142 & $(9 \%)$ & \multicolumn{2}{l}{$1412(87 \%)$} & 64 & $(4 \%)$ \\
$452(37 \%)$ & - & & 0 & $(0 \%)$ & 767 & $(63 \%)$ \\
$1387(100 \%)$ & 0 & $(0 \%)$ & - & & 0 & $(0 \%)$ \\
$235(14 \%)$ & $1504(86 \%)$ & 0 & $(0 \%)$ & - &
\end{tabular}

OC3

Right Nerve (2613)

Left Tract (3133)

Right Tract (3332)

$235(14 \%) \quad 1504(86 \%) \quad 0 \quad(0 \%) \quad-$

\begin{tabular}{llllll} 
& & Left Nerve & Right Nerve & Left Tract & \multicolumn{1}{l}{ Right Tract } \\
\cline { 3 - 6 } Left Nerve & $(7141)$ & - & $1300(36 \%)$ & $2114(59 \%)$ & 182 \\
Right Nerve & $(6752)$ & $1373(37 \%)$ & - & $166(5 \%)$ & $2135(58 \%)$ \\
Left Tract & $(7814)$ & $4738(75 \%)$ & $287(5 \%)$ & - & $1322(20 \%)$ \\
Right Tract & $(8690)$ & $147(2 \%)$ & $5029(81 \%)$ & $1054(17 \%)$ & - \\
\hline
\end{tabular}

Table 1: Absolute numbers and percentages (in brackets) of streamlines tracked from a given seed location (row) that passes through a given target location (column) for all three OCs. Percentages are relative to all streamlines that reached a location (i.e. relative to the sum of streamlines in a row). The absolute number of lines tracked from the seed location (longer than $2 \mathrm{~mm}$ ) are listed behind it in brackets. 


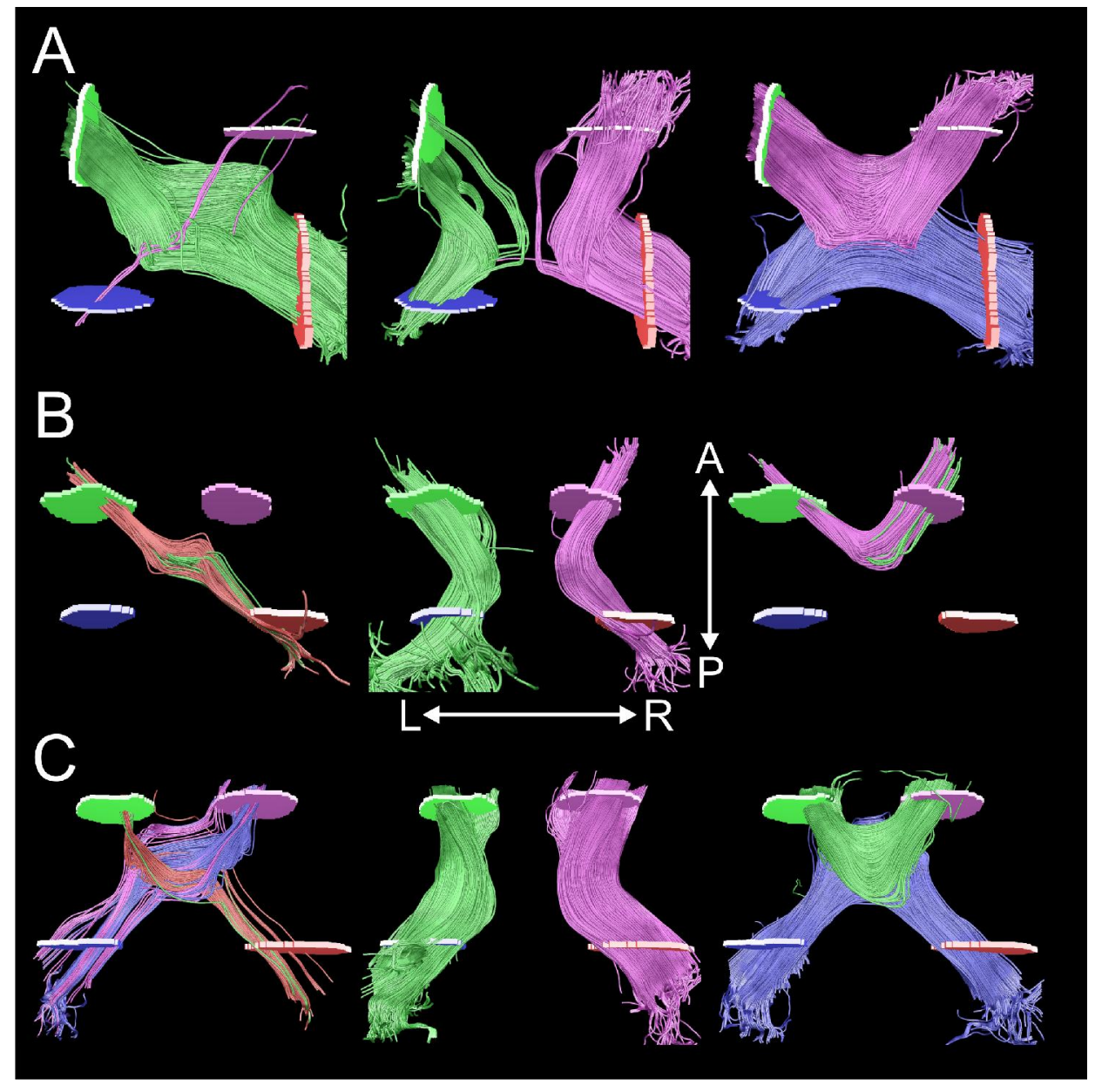

85

Figure 5: Selected fiber tracking results for OC1 in A (top row), OC2 in B (middle row), and OC3 in $C$ (bottom row). Seed regions are shown for the left optic nerve (in green), the right optic nerve (in purple), the left optic tract (in blue), and the right optic tract (in red). All displayed tracking results are color according to the seed region from which they were initiated. Post-selection for passage through a second VOI (effectively an AND operation) was performed to group tractography results with the same start and end regions. Left column shows stream-lines crossing both the horizontal and vertical midline, middle column shows stream-lines connecting optic nerve and optic tract on the same side, right column shows stream-lines connecting either optic nerves or optic tracts on both sides. 
Figure 5 and Table 1 show that almost all connections between VOls are tracked (including the non-veridical connections between optic nerves) though in varying relative proportions. On the whole symmetry exists in the amount of fibers tracked in either direction between two VOls (i.e. a similar amount of fibers are tracked from $A$ to $B$ and from $B$ to $A$ ). Fibers crossing from optic nerve to contralateral optic tract (or vice-versa) that should be the most numerous, are found the least. Whereas their proportion of all fibers tracked from a VOI in the optic nerve or tract should be around $75 \%$, it is only found to be in the range of $0-10 \%$ for OC2 and OC3. For OC1, it is about $30 \%$, but only for connections between left nerve and right tract (see Figure 5, left column). Connections between optic nerve and ipsilateral optic tract, expected in a proportions of about $25 \%$, are overrepresented with proportions of tracked fibers between $19 \%$ and $42 \%$ for OC $1,63 \%$ and $100 \%$ for OC2, and $58 \%$ and $81 \%$ for OC3 (see Figure 5, middle column). Connections between the optic nerves that should not exist, are tracked in considerate numbers with proportions of $9 \%$ to $58 \%$ of all fibers tracked from the optic nerve VOls (see Figure 5, right column). Finally, the proportion of fibers that run through both the optic tracts, connecting the optic tract VOls also seem to be overrepresented in OC1 at $49 \%$ to $73 \%$ and OC3 at $17 \%$ to $20 \%$, but are completely absent in OC2. Overall, fibers crossing from optic nerve to contralateral optic tract, that should be the most numerous, are underrepresented, whereas fibers connecting VOls on the same side of the horizontal or vertical midline are heavily overrepresented.

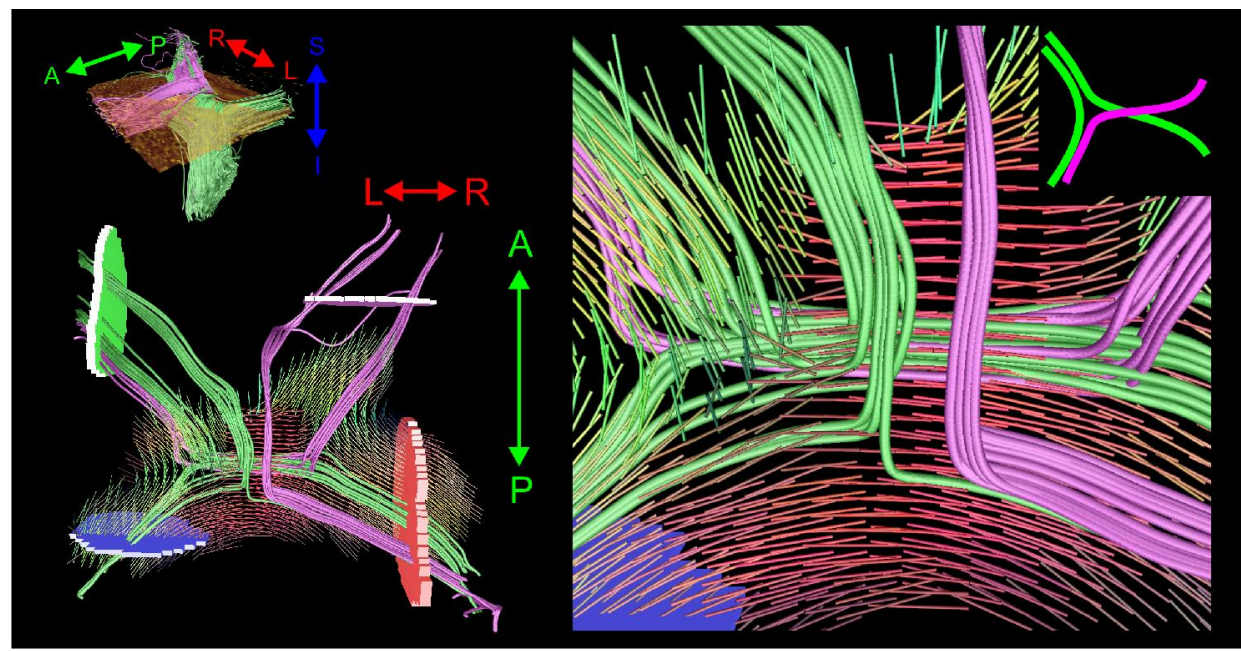

Figure 6: A detail-rendering (from above) of selected tractography results for $\mathrm{OC} 1$ along with a direction color-coded rendering of local primary diffusion directions in a near-axial slice. Coloring of seed regions and tractography results as before. Inset at the top-right shows a schematic of the expected true course of the local fibers. Note that only parts of the rendered tracts run through the selected slice. 
Detail renderings of selected fibers of the above classes where they pass the critical high curvature regions, overlaid on primary eigenvector renderings, are very informative in clarifying the pattern of connectivity described above. Figure 6 shows selected trackings of both the left and the right optic nerve VOI for OC1. The selected streamlines of the left optic nerve VOI pass through either the left or right optic tract VOls. Although these connections are likely to reflect existing fibers, the path traced by these tracked streamlines is unlikely to be veridical. The tracings terminating in the contralateral optic tract were initiated in the temporal part of the optic nerve where fibers are situated that generally pass to the ipsilateral optic tract. The tracing terminating in the ipsilateral tract were initiated in the nasal part of the left optic nerve from where fibers generally cross to the contralateral optic tract. These seemingly non-veridical tracings seem to arise from the problematic region enlarged in Figure 6, where (most likely) fibers passing from the left optic nerve to the left and right optic tracts diverge, and fibers from the right optic nerve en-route to the left optic tract converge with left optic nerve fibers with the same target. Furthermore, slightly left (medial) of this 'three-fold kissing' the left and right decussating nerve tracts cross each other (see inset at the top-right of Figure 6). A similar critical branching point should exist on the other side of the midline. The selected streamlines originating from the right optic nerve VOI pass through either the left optic nerve VOI or the right optic tract VOI, neither of which is likely to be a veridical tract. The 'eye-to-eye' connection in particular seems to be the result of a wrong turn made at both the left and right critical branching points.

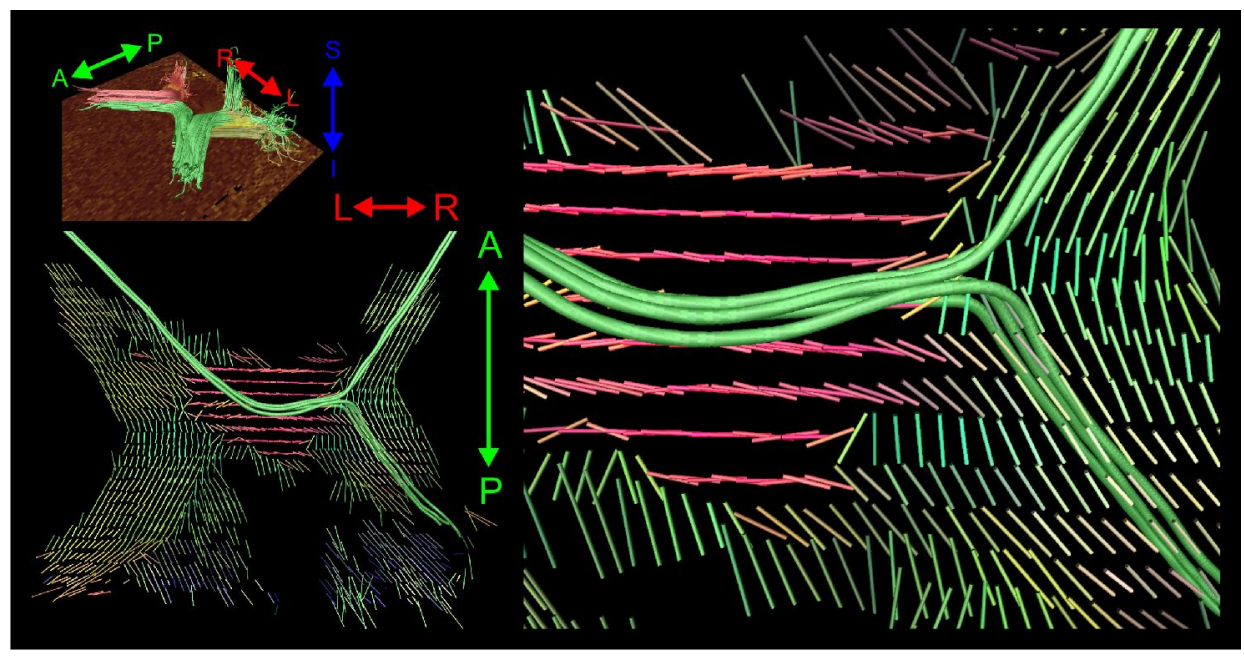

Figure 7: A detail-rendering (from above) of selected tractography results for OC2 along with a direction color-coded rendering of local primary diffusion directions in a near-axial slice. Coloring of seed regions and tractography results as before. Note that only parts of the rendered tracts run through the selected slice. 
Figure 7 shows a detail of selected trackings for OC2 initiated from the medial part of the left optic nerve VOI. Two sets of streamlines are depicted that initially follow a very similar path posteriorly through the optic nerve and then curving medially into the central chiasm. However, at the branch point on the contralateral side of the central chiasm the tracked bundle splits into one group of tracts that turns posteriorly into the right optic tract, and a second group that turns anteriorly into the right optic nerve. It can be seen that the turn at the branching point is determined by the relative spatial position and the precise angle at which it is entered, owing to the purely local information used by the tracking algorithm and the slight inertia exercised by the previous tracking direction.

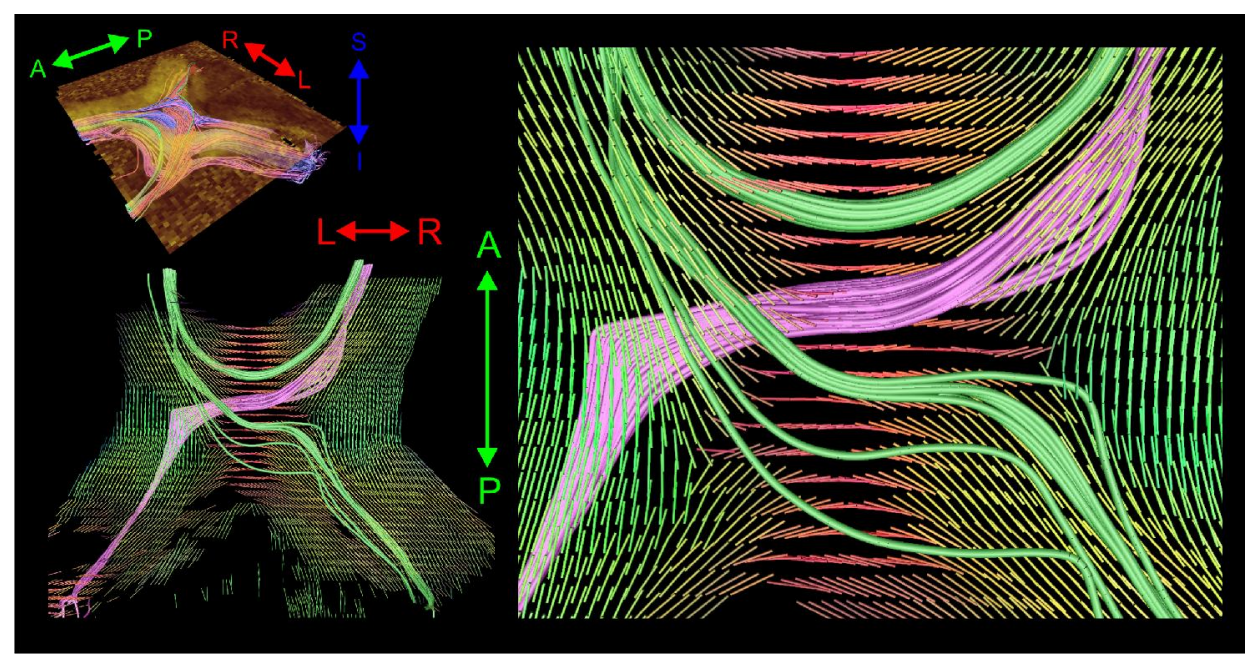

Figure 8: A detail-rendering (from above) of selected tractography results for OC3 along with a direction color-coded rendering of local primary diffusion directions in a near-axial slice. Coloring of seed regions and tractography results as before. Note that only parts of the rendered tracts run through the selected slice.

Figure 8 shows a detail rendering of streamlines originating in the left and right optic tract VOIs of OC3. It shows seemingly veridical trackings between the optic nerves and contralateral tracts, and incorrect tracked fibers connecting the left and right optic nerve. The latter are seen to arise from the left-to-right orientation of the primary eigenvectors at the midline of the central chiasm where left and right optic nerve projections cross, as discussed above. Instead of continuing their course posteriorly across the midline, the left optic nerve streamlines are deflected to a path straight across the midline and then erroneously follow the right optic nerve projections retrogradely toward the right eye. The tracked fibers between the optic nerves and contralateral tracts curve similarly towards a right-left orientation near the midline but, importantly maintain a slightly diagonal anteriorposterior course, curving towards the anterior-posterior direction again on the other side of the midline. Note also that these two streamline group cross each other (slightly offset in the inferior-superior dimension) at a reasonable large angle and do not pass through the critical branching points. 
$1 \times 2 \times 2(312.5 \mu \mathrm{m})$

Left Nerve

(4053)

Right Nerve

\begin{tabular}{llll} 
Left Nerve & Right Nerve & Left Tract & Right Tract \\
\hline- & $596(31 \%)$ & $1253(65 \%)$ & $75(4 \%)$ \\
$700(34 \%)$ & - & $85(4 \%)$ & $1299(62 \%)$
\end{tabular}

$2 \times 4 \times 4(625 \mu \mathrm{m})$

Left Nerve

Right Nerve

\begin{tabular}{llll} 
Left Nerve & Right Nerve & Left Tract & Right Tract \\
\hline- & $361(28 \%)$ & $918(70 \%)$ & $33(2 \%)$ \\
$466(32 \%)$ & - & $63(4 \%)$ & $941(64 \%)$
\end{tabular}

$4 \times 8 \times 8(1250 \mu m)$

Left Nerve

Left Nerve

\begin{tabular}{lll} 
Right Nerve & Left Tract & Right Tract \\
\hline $143(18 \%)$ & $656(82 \%)$ & $3(0.3 \%)$ \\
- & $12(1 \%)$ & $945(83 \%)$ \\
\hline
\end{tabular}

Table 2: Absolute numbers and percentages (in brackets) of streamlines tracked from a given seed location (row) that passes through a given target location (column) for the three downsampled versions of OC3. Percentages are relative to all streamlines that reached a location (i.e. relative to the sum of streamlines in a row). The absolute number of lines tracked from the seed location (longer than $2 \mathrm{~mm}$ ) are listed behind it in brackets.

The effects of spatial resolution on the results was investigated by performing the same analysis on down-sampled versions of OC3. OC3 was selected because it showed the most consistent percentage of tracked fibers crossing over the midline from optic nerve to optic tract. Absolute numbers and relative percentages of tracked fibers with the left or right optic nerve $\mathrm{VOI}$ as the seeding region for the down-sampled datasets are listed in Table 2. Observing the proportion of tracked fibers for OC3 with a given termination area with progressively coarser resolution in tables 1 and 2, two observations can be made. First, there is a clear tendency for the under-representation of fibers crossing from optic nerve to contralateral VOI to get more severe with decreasing resolution. The percentage of optic-nerve streamlines crossing to the contralateral optic tract VOI in the original data is about $5 \%$. It decreases to $4 \%, 3 \%$, and $1 \%$ in the down-sampled datasets. Second, an increase with coarser resolution in the over-representation of ipsilateral nerve-to-tract connections seems evident. Where the percentage of opticnerve streamlines passing to the ipsilateral optic tract VOI in the original data is about $60 \%$, it increases to $65 \%, 70 \%$, and $80 \%$ in the down-sampled datasets. This increase in streamlines that remain on one side of the midline is gained not only from decreased tracings from nerve to contralateral tract, but also from a decreasing proportion of nerveto-nerve connections. All streamlines resulting from the fiber trackings from the optic nerve VOls, passing through the contralateral optic tract VOIs are shown in Figure 9. Besides the decreasing number of the crossing nerve-to-tract connections with 


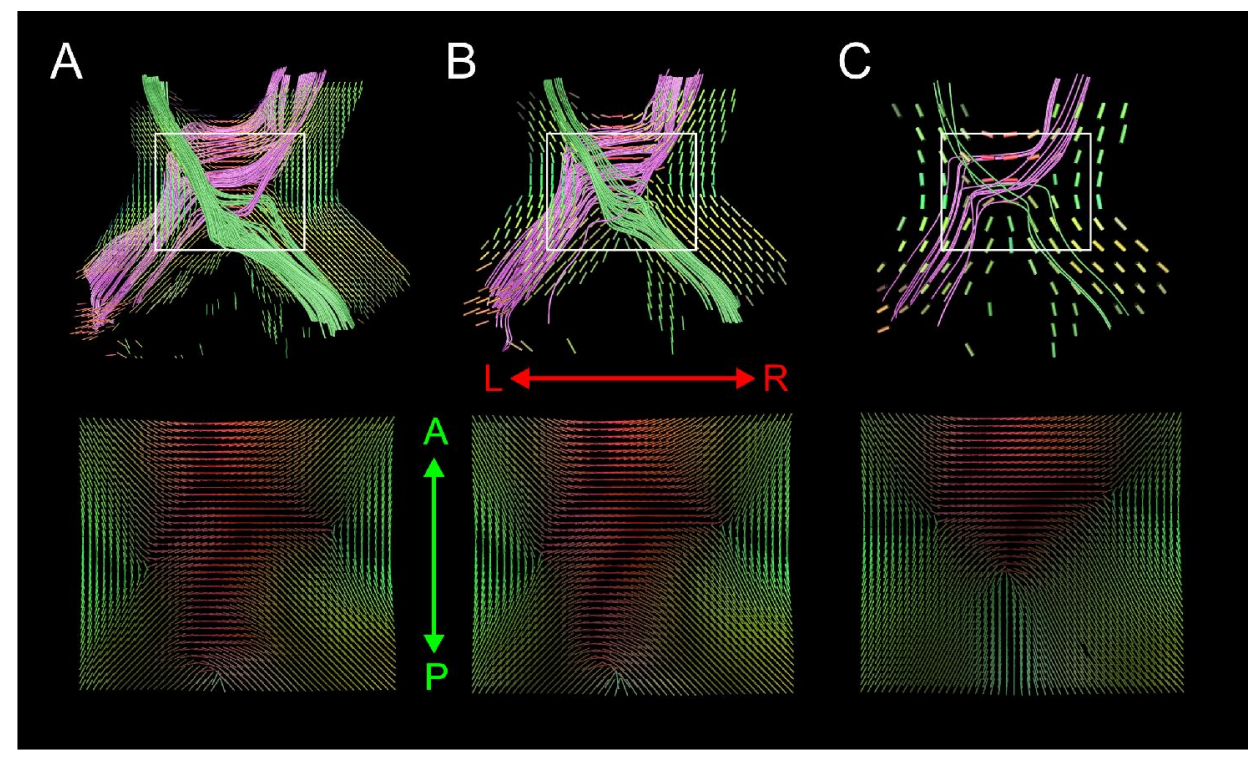

Figure 9: Top-row: results for fiber tracking from the optic nerve VOIs, passing through the contralateral optic tract VOls for each of the down-sampled versions of the OC3 dataset. Bottom-row: A resampling (by interpolation) of a selected part of each dataset to the original resolution of $156 \mu \mathrm{m}$, to illustrate the loss of spatial detail at a progressively coarser resolution.

decreasing resolution, also evident from the decreasing percentages in Table 2, The exact path followed by those connections also seems to alter. The crossing connections in totality seem to pass through a successively narrower portion of the central chiasm. Furthermore, the tracts that remain at the lowest resolutions predominantly have a straight left-to-right path through the central chiasm. Fibers curving back towards the contralateral optic nerve, before bending sharply posteriorly towards the contralateral optic tract are soon smoothed to a seemingly more direct (but not more veridical, see Figure 1) left-to-right path throught the chiasm. Interpolation of the down-sampled datasets to the original resolution for easy visual comparison shows partial volume averaging in the coarser resolution datasets to be the cause of these results. The subtle anterior-posterior slope of the fibers crossing through the central chiasm is progressively averaged to an almost pure left-to-right orientation of the eigenvectors over more and more of the area surrounding the midline in the lower resolution datasets. Furthermore, partial volume averaging of the central chiasm with parts of the incoming and outgoing optic nerves and tracts causes the narrowing of the representation of the central chiasm at lower spatial resolution. 


\section{Discussion}

High resolution diffusion tensor imaging of the human optic chiasm was performed ex vivo at ultra-high field to validate of the diffusion tensor model and streamline tractography algorithms. The complex micro-structure of the optic chiasm where complex fiber configurations are present, such as kissing, crossing, convergence and sharp curves, was well characterized at the acquired resolution. Specifically, the regions within the optic chiasm where such problematic configurations occur could be localized with high spatial accuracy. Two zones within the central chiasm, in particular, contain most of the complex fiber configurations. The first is located at the lateral-most aspects of the central chiasm where it intersects with the medial aspects of the optic nerves and tracts, and is highlighted by a sharp reduction in the $C_{l}$ coefficient, indicating non-prolate estimated tensor shapes there. These zones proceed bilaterally on each side of the central chiasm from the anterior and ventral aspect to the posterior and dorsal aspect of the lateral central chiasm, forming diagonal bands (see Figures 2, 3, and 4). Primary eigenvector directions suggest that in these zones, fibers passing from the optic nerve to the ipsilateral and contralateral optic tracts diverge, and fibers from the optic nerve on the other side crossing oppositely converge with the fibers with the same target that stayed on one side of the midline, forming a critical branching point in the form of a 'three-fold kissing' (see inset in Figure 6). The second region of complex fiber architecture is the expected crossing of tracts from the two optic nerves decussating to their contralateral optic tracts in the central optic chiasm. Most decussating fibers seem to curve, often sharply at the critical branching points, to follow a more medio-lateral direction through the central chiasm, crossing fibers from the opposite optic nerve at a shallow angle (see, again, inset in Figure 6). The diffusion tensor model is unable to accurately represent this crossing, resulting from its gaussian approximation of the diffusion profile. Most likely the average direction of the two crossing fibers is what is represented at the crossing sites in the central chiasms.

Streamline tractography by a line propagation algorithm was used to investigate to which degree the known macroscopic connectivity through the optic chiasm could be reproduced. Fibers crossing from optic nerve to contralateral optic tract (or vice-versa) should be the most numerous, but were found the least. Whereas their proportion of all fibers tracked from a VOI in the optic nerve or tract should be around $75 \%$, it was only found to be in the range of $0-10 \%$ for OC2 and OC3. Overall, fibers crossing from optic nerve to contralateral optic tract, that should be the most numerous, are underrepresented, whereas fibers connecting VOIs on the same side of the horizontal or vertical midline are heavily overrepresented. The overrepresentation of tracts connecting optic nerve with the ipsilateral optic tracts and connections between the two nerves or between the two tracts are mainly the result of problems of the tracking algorithm at the two regions of complex fiber architecture. For the tracked fibers that cross through the chiasm, the turn made at the critical branching regions is determined by the relative spatial position and the precise angle at which it is entered. This, in turn, is determined by the way these streamlines pass through the crossing regions in the central chiasm. An example of the resulting inaccuracies is shown in Figure 8, where tracked fibers erroneously connect the two optic nerves. Instead of continuing their course posteriorly across the midline, the left optic nerve streamlines are deflected to a path straight across 
the midline and then erroneously follow the right optic nerve projections retrogradely toward the right eye. Despite the suboptimal performance of DTI-tractography by line propagation algorithms on these high resolution datasets, down-sampling showed importance of high spatial resolution acquisitions. The under-representation of fibers crossing from optic nerve to contralateral VOI, and the over-representation of ipsilateral nerve-to-tract connections got more severe with decreasing resolution. Moreover, the actual paths traced out in the down-sampled datasets for the remaining crossing fiber were seen to be less veridical. These distorted paths seem to be caused by the distorted tensor fields resulting from partial volume averaging in the lower resolution datasets.

Over all, this study shows the post-mortem investigation of fixed tissue samples with DTI at high field strengths to be an attractive candidate for the validation of the diffusion tensor model and tractography algorithms and the effects of spatial resolution on their performance. Moreover, even without tractography, the mapping of characteristics of local diffusion in these datasets can potentially increase our knowledge of the detailed 3$\mathrm{D}$ anatomy of neural structures, such as the human optic chiasm. The main limitation of the current study is the lack of a ground truth for the exact true paths followed by axonal fiber bundles through the chiasm. Although much of the macroscopic connection through the chiasm and even the general shapes of the paths through the chiasm are known (see Figure 1), decisions on the correctness of discrete tracked streamlines in individual chiasms needs more information. A tracked path could seem compatible with prior knowledge and the data at hand, yet be non-veridical. Combination of high-resolution imaging with more classical ways of determining anatomical connectivity, such as tracer injections, seems the most suitable way of providing a gold standard for the validation tractography results. Since actively transported tracers, such as retro-virusses, will be unsuitable for post-mortem tracer studies, passively diffusing tracers will have to be used. In particular, injection of passively diffusing dyes, at discrete tissue sites with different axonal projection will be of great value for the validation of tractography results.

Some of the obvious errors made by the DTI-based line propagation algorithm clearly arise from the inadequacy of the diffusion tensor model to represent complex fiber architecture, such as fiber crossings, and from the constraint on the tractography algorithm to use only local information. Complex fiber configurations, such as crossings, when captured in a single voxel, will give rise to non-gaussian diffusion profiles due to partial volume averaging (Alexander et al., 2002; Frank, 2001, 2002; Tuch et al., 2002). The diffusion tensor is unable to represent such profiles with more than a single defined maximum, as illustrated, for example, in the case of the low-angle crossing in the central optic chiasm. Recently, alternative models, capable of representing non-gaussian diffusion profiles in voxels with complex fiber architectures have been proposed (Assaf et al., 2004; Jansons and Alexander, 2003; Liu et al., 2004; Ozarslan and Mareci, 2003; Tournier et al., 2004; Tuch, 2004; Tuch et al., 2003; Wedeen et al., 2005). These estimation of these models require high angular resolution diffusion imaging (HARDI) acquisitions, where the angular resolution of the diffusion direction sampling is greatly increased, from six directions, needed for estimation of the diffusion tensor, to tens or even hundreds of directions. It will be interesting to investigate whether these nongaussian diffusion models are better capable of representing the diffusion profiles in regions of complex architecture in the optic chiasm. Furthermore, it will be informative to 
evaluate tractography results based on these models, to verify whether a more accurate local representation of diffusion leads to more accurate tracked global connections.

The constraint of line-propagation algorithms to only use local information at the current point of tracking has recently led to two further forms of developments in tractography algorithms. Recent developments in tractography algorithms have aimed at removing two constraints of the line propagation algorithm: its use of only local information at the current tracking point, and its lack of providing any kind of reliability measure for a resulting streamline. First, some developments have taken place in tractography algorithms that minimize a more global fit of a streamline to the discrete estimated tensor field through which it passes, for instance by estimating geodesics in the tensor field considered as a Riemannian manifold (Lenglet et al., 2004), or by using the Metropolis algorithm, a simulated annealing approach that iteratively maximizes the fit of the entire tract to the data (Tuch, 2002). This could make the resulting streamlines less prone to errors at critical points such as crossings or regions of high curvature. Second, many recent investigation have investigated so-called probabilistic tractography algorithms that provide for a given seedpoint not a single most likely terminal point of connection along with a joining tract, but rather a 3-D map of connection probability. The idea is to provide a probabilistic index of connectivity (PICo) that quantifies the likelihood of an anatomical connection linking two points given the estimated field of local diffusion profiles (Parker et al., 2003). Such probabilistic tractography algorithms generally involve either a monte carlo sampling scheme where many repetitions of a diffusing particle following a noise-perturbed path are summarized in a map of relative 'particle-visits' (Koch et al., 2002; Parker and Alexander, 2003; Parker et al., 2003), or some form of a front-evolution algorithm that will assign arrival-times to all points in the volume, interpretable as the inverse of connection-probability (Campbell et al., 2005; Jackowski et al., 2005; Parker et al., 2002).

Investigation of the performance of these alternative algorithms on high resolution datasets such as those used here will be of considerable interest. Line propagation algorithms use only local information at each step through the data, which can lead to rapid accumulation of errors, particularly when critical regions (crossings, high curvature) have to be passed. Potentially, algorithms that maximize the global fit of a trajectory are less prone to errors at critical regions when a wrong path through a critical region will decrease the fit to the surrounding data. It should be noted, however, that the constraints of surrounding data do not always resolve the correct passage through a critical region, as is the case, for instance in a kissing vs. crossing situation. The application of probabilistic algorithms to the current datasets could be of interest to investigate whether the connection likelihoods they provide would reflect the relative proportions of streamline terminations for a given seed VOI reported here. The evolution of the path of a randomly perturbed particle or a propagating front is generally constrained by the same local information at the point of propagation (shapes of estimated tensors) and the same constraints (anisotropy thresholds, angle thresholds) as the standard deterministic line propagation algorithms are. Therefore, switching from a deterministic to a stochastic tractography algorithm per se will not necessarily yield an improvement in the accuracy of the tracking results.

This study has shown that the post-mortem investigation of fixed tissue samples with DTI at high field strengths is an attractive candidate for the validation of MR-based 
diffusion models and tractography algorithms. Furthermore, the effects of spatial resolution and the varying degrees of partial volume averaging of complex fiber architecture on the performance of these methods could be investigated. Diffusion MR based tractography has the potential to provide quantitative measures of axonal connectivity in the living human brain. Future investigations of the performance of MRbased diffusion models and tractography algorithms on fixed tissue samples, in combination with passively diffusing tracers, is of great importance in the validation of this endeavour. 


\section{References}

Alexander AL, Hasan KM, Lazar M, Tsuruda JS, Parker DL (2001) Analysis of partial volume effects in diffusion-tensor MRI. Magn Reson Med 45: 770-780.

Alexander DC, Barker GJ, Arridge SR (2002) Detection and modeling of non-Gaussian apparent diffusion coefficient profiles in human brain data. Magn Reson Med 48: 331-340.

Assaf Y, Freidlin RZ, Rohde GK, Basser PJ (2004) New modeling and experimental framework to characterize hindered and restricted water diffusion in brain white matter. Magn Reson Med 52: 965-978.

Basser PJ, Mattiello J, LeBihan D (1994) Estimation of the effective self-diffusion tensor from the NMR spin echo. J Magn Reson B 103: 247-254.

Basser PJ, Pajevic S, Pierpaoli C, Duda J, Aldroubi A (2000) In vivo fiber tractography using DTMRI data. Magn Reson Med 44: 625-632.

Campbell JS, Siddiqi K, Rymar VV, Sadikot AF, Pike GB (2005) Flow-based fiber tracking with diffusion tensor and q-ball data: Validation and comparison to principal diffusion direction techniques. Neuroimage.

Catani M, Howard RJ, Pajevic S, Jones DK (2002) Virtual in vivo interactive dissection of white matter fasciculi in the human brain. Neuroimage 17: 77-94.

Conturo TE, Lori NF, Cull TS, Akbudak E, Snyder AZ, Shimony JS, McKinstry RC, Burton H, Raichle ME (1999) Tracking neuronal fiber pathways in the living human brain. Proc Natl Acad Sci U S A 96: 10422-10427.

Frank LR (2001) Anisotropy in high angular resolution diffusion-weighted MRI. Magn Reson Med 45: 935-939.

Frank LR (2002) Characterization of anisotropy in high angular resolution diffusion-weighted MRI. Magn Reson Med 47: 1083-1099.

Guilfoyle DN, Helpern JA, Lim KO (2003) Diffusion tensor imaging in fixed brain tissue at 7.0 T. NMR Biomed 16: 77-81.

Hagmann P, Thiran JP, Jonasson L, Vandergheynst P, Clarke S, Maeder P, Meuli R (2003) DTI mapping of human brain connectivity: statistical fibre tracking and virtual dissection. Neuroimage 19: 545-554.

Jackowski M, Kao CY, Qu M, Constable RT, Staib LH (2005) White matter tractography by anisotropic wavefront evolution and diffusion tensor imaging. Med Image Anal 9: 427-440.

Jansons KM, Alexander DC (2003) Persistent Angular Structure: new insights from diffusion MRI data. Dummy version. Inf Process Med Imaging 18: 672-683.

Jones DK, Simmons A, Williams SC, Horsfield MA (1999) Non-invasive assessment of axonal fiber connectivity in the human brain via diffusion tensor MRI. Magn Reson Med 42: 37-41.

Koch MA, Norris DG, Hund-Georgiadis M (2002) An investigation of functional and anatomical connectivity using magnetic resonance imaging. Neuroimage 16: 241-250.

Lazar M, Weinstein DM, Tsuruda JS, Hasan KM, Arfanakis K, Meyerand ME, Badie B, Rowley HA, Haughton V, Field A, Alexander AL (2003) White matter tractography using diffusion tensor deflection. Hum Brain Mapp 18: 306-321.

Lenglet C, Deriche R, Faugeras O (2004) Inferring white matter geometry from diffusion tensor MRI: Application to connectivity mapping. In: (Pajdla T, Matas J, eds.) 8th European Conference on Computer Vision. Prague, Czech Republic: Springer--Verlag. 
Liu C, Bammer R, Acar B, Moseley ME (2004) Characterizing non-Gaussian diffusion by using generalized diffusion tensors. Magn Reson Med 51: 924-937.

Lori NF, Akbudak E, Shimony JS, Cull TS, Snyder AZ, Guillory RK, Conturo TE (2002) Diffusion tensor fiber tracking of human brain connectivity: aquisition methods, reliability analysis and biological results. NMR Biomed 15: 494-515.

Mori S, Crain BJ, Chacko VP, van Zijl PC (1999) Three-dimensional tracking of axonal projections in the brain by magnetic resonance imaging. Ann Neurol 45: 265-269.

Mori S, Kaufmann WE, Davatzikos C, Stieltjes B, Amodei L, Fredericksen K, Pearlson GD, Melhem ER, Solaiyappan M, Raymond GV, Moser HW, van Zijl PC (2002) Imaging cortical association tracts in the human brain using diffusion-tensor-based axonal tracking. Magn Reson Med 47: 215-223.

Ozarslan E, Mareci TH (2003) Generalized diffusion tensor imaging and analytical relationships between diffusion tensor imaging and high angular resolution diffusion imaging. Magn Reson Med 50: 955-965.

Pajevic S, Pierpaoli C (1999) Color schemes to represent the orientation of anisotropic tissues from diffusion tensor data: application to white matter fiber tract mapping in the human brain. Magn Reson Med 42: 526-540.

Parker GJ, Alexander DC (2003) Probabilistic Monte Carlo based mapping of cerebral connections utilising whole-brain crossing fibre information. Inf Process Med Imaging 18: 684-695.

Parker GJ, Haroon HA, Wheeler-Kingshott CA (2003) A framework for a streamline-based probabilistic index of connectivity (PICo) using a structural interpretation of MRI diffusion measurements. J Magn Reson Imaging 18: 242-254.

Parker GJ, Stephan KE, Barker GJ, Rowe JB, MacManus DG, Wheeler-Kingshott CA, Ciccarelli O, Passingham RE, Spinks RL, Lemon RN, Turner R (2002) Initial demonstration of in vivo tracing of axonal projections in the macaque brain and comparison with the human brain using diffusion tensor imaging and fast marching tractography. Neuroimage 15: 797-809.

Pierpaoli C, Basser PJ (1996) Toward a quantitative assessment of diffusion anisotropy. Magn Reson Med 36: 893-906.

Poupon C, Clark CA, Frouin V, Regis J, Bloch I, Le Bihan D, Mangin J (2000) Regularization of diffusion-based direction maps for the tracking of brain white matter fascicles. Neuroimage 12: 184-195.

Press W, Teukosky S, Vetterling W, Flannery B (2002) Numerical Recipes in C++: the art of scientific computing. Cambridge: Cambridge University Press.

Standring S, ed. 2005. Gray's Anatomy: The Anatomical Basis of Clinical Practise, 39 ed. Edinburgh, London: Elsevier Churchill Livingstone.

Sun SW, Neil JJ, Liang HF, He YY, Schmidt RE, Hsu CY, Song SK (2005) Formalin fixation alters water diffusion coefficient magnitude but not anisotropy in infarcted brain. Magn Reson Med 53: 1447-1451.

Sun SW, Neil JJ, Song SK (2003) Relative indices of water diffusion anisotropy are equivalent in live and formalin-fixed mouse brains. Magn Reson Med 50: 743-748.

Tournier JD, Calamante F, Gadian DG, Connelly A (2004) Direct estimation of the fiber orientation density function from diffusion-weighted MRI data using spherical deconvolution. Neuroimage 23: 1176-1185.

Tuch DS (2002) Diffusion MRI of Complex Tissue Structure. In: Division of Health Sciences and Technology. Boston: MASSACHUSETTS INSTITUTE OF TECHNOLOGY.

Tuch DS (2004) Q-ball imaging. Magn Reson Med 52: 1358-1372. 
Tuch DS, Reese TG, Wiegell MR, Makris N, Belliveau JW, Wedeen VJ (2002) High angular resolution diffusion imaging reveals intravoxel white matter fiber heterogeneity. Magn Reson Med 48: 577-582.

Tuch DS, Reese TG, Wiegell MR, Wedeen VJ (2003) Diffusion MRI of complex neural architecture. Neuron 40: 885-895.

Wakana S, Jiang H, Nagae-Poetscher LM, van Zijl PC, Mori S (2004) Fiber tract-based atlas of human white matter anatomy. Radiology 230: 77-87.

Wedeen VJ, Hagmann P, Tseng WY, Reese TG, Weisskoff RM (2005) Mapping complex tissue architecture with diffusion spectrum magnetic resonance imaging. Magn Reson Med 54: 1377-1386.

Westin CF, Maier SE, Mamata H, Nabavi A, Jolesz FA, Kikinis R (2002) Processing and visualization for diffusion tensor MRI. Med Image Anal 6: 93-108.

Xue R, van Zijl PC, Crain BJ, Solaiyappan M, Mori S (1999) In vivo three-dimensional reconstruction of rat brain axonal projections by diffusion tensor imaging. Magn Reson Med 42: 11231127. 
General Discussion 
This thesis has investigated the study of functional integration in the brain, by application of Granger causality to fMRI signals to investigate effective connectivity, and the use of diffusion tensor imaging and fiber tracking to investigate anatomical connectivity. Both techniques were considered as complements to the current fMRI effective connectivity methods that presuppose a structural model of regions involved in a cognitive task or process and the anatomical connections between them. fMRI Granger causality mapping was proposed as a data-driven way to explore regions in the brain that interact with a selected reference region as measured from their functional activity. Diffusion tensor imaging and fiber tracking were investigated as an approach to investigate anatomical connections between cortical and subcortical regions in vivo. In this chapter, both methods are evaluated in this context, possibilities for future improvements to the techniques are discussed, and a more general outlook on possible ways to integrate these methods into functional integration studies is provided.

\section{Granger causality mapping}

Granger causality mapping (GCM) was proposed in this thesis as an approach to explore directed influences between neuronal populations in fMRI data. The method does not rely on a priori specification of a 'structural' or 'anatomical' model that contains preselected regions and connections between them. This distinguishes it from other effective connectivity approaches that aim at testing or contrasting specific hypotheses about neuronal interactions within an assumed anatomical model. Such pre-specified models are very useful, when specific hypotheses about neuronal interactions in a wellknown network are to be tested. However, inference on a hypothesis concerning part of the specified network is very sensitive to misspecification of the model. The omission of areas or structures that mediate influences or form an additional source of influence can easily lead to spurious interactions. Furthermore, in early stages of investigation specific hypotheses about the exact network underlying performance of a cognitive task might not be readily available.

Instead, GCM relies on the concept of Granger causality to define the existence and direction of influence between two stochastic time-series purely on the basis of temporal precedence in their interdependency. As an exploratory method, Granger causality mapping can form an important complement to these hypothesis driven methods in helping to formulate directed graph models of regions and their interactions. Statistical inference for Granger causality maps was extended to the random effects level such that the consistency of explored regional interactions over a group of subjects could be assessed. In chapter 2, this technique was shown to be useful in identifying the interactions of premotor and parietal cortex in switching visuomotor association tasks, which were largely unknown to date. Both in chapter 1 and 2, some of the important considerations in using and interpreting GCMs were discussed. These considerations are summarized in four main issues: 1) The effect of intervening hemodynamics in fMRI, 2) The effect of temporal sampling, 3) The question of spurious causality, and 4) The case of bidirectional interactions.

Because relative temporal precedence in the blood oxygenation level dependent (BOLD) signals of two regions is used in GCM to evaluate existence and directions of causal influence, note must be taken of the possible disturbing effect on GCM inferences 
of differences in the local hemodynamics over the brain. Of primary concern here is the possibility of a systematic difference in the lag of the hemodynamic response between different brain structures. Such a systematic difference could yield spurious influences. Therefore, it was argued in chapter 1 that Granger causal influences in fMRI data should show modulation by experimental parameters manipulating cognitive context to assign inferred influences to neuronal interactions, not to differences in hemodynamics. The reasoning is that structural differences in hemodynamics persist over different conditions or contexts, so that any observed systematic variation with condition or context, should be due to changes in the neuronal population interactions. Thus, in the presence of such experimentally modulated influence, one can more reliably conclude that the measured influences reflect true neuronal interactions. In chapter 2, statistical inference for Granger causality maps was extended to the random effects level such that the consistency of explored regional interactions over a group of 15 subjects could be assessed. Consistent influences between regions were shown on the group level, and, moreover, significant modulation of these influences by experimental factors was shown at the random-effects level. This shows that GCM can be a very powerful addition to current effective connectivity techniques, but only if applied in consideration of the confounding hemodynamics.

The simulations in chapter 1 showed the effect of temporal sampling on the power of the GCM method to resolve temporally delayed interactions to be considerable. In principle, the influence between neuronal populations were shown to be detectable in the $\mathrm{fMRI}$ signal, even if the timescale and delay of the influence is smaller than the interval at which the data is sampled. However, the sensitivity to such interactions decreased rapidly with increasing sampling interval. Decreasing the sampling interval for a simulated influence of given strength and delay, generally increased statistical detection power. In the simulated system, a simple abstraction of realistic neuronal population interactions and the effect of hemodynamics, most power was gained by decreasing the sampling interval from $1 \mathrm{sec}$. to $0.5 \mathrm{sec}$. This raises the question of what the optimal TR (i.e. sampling interval) is for an fMRI investigation employing GCM. The short answer to that question, taking account only of the detection power available by GCM, would be to make it as short as possible. However, in general there are additional considerations. The simulations showed that the temporal resolution needed to reach a given detection power is dependent on the strength and delay of the interactions of interest. Thus, the minimal temporal resolution needed is dependent on the task, the regions, and the interactions of interest. Moreover, temporal resolution, spatial resolution, spatial coverage and signal-to-noise ratio (SNR) are adjustable in the MR sequences for fMRI, but only at a relative mutual cost. Temporal resolution can be increased at the cost of a decrease in spatial resolution and/or spatial coverage. For instance, to obtain relatively high temporal resolution, one could limit the acquisition to only a few slices, which can be recorded in a few hundreds of milliseconds. This has the potential to increase the detection power of GCM for relatively fast neuronal interactions. However, it would also limit the field of view on activity to only a small part of the brain. Thus, the potential for GCM to map regions interacting with a selected reference region will be greatly reduced to those in the chosen volume of coverage. A prior investigation with a full field of view would have to be performed to determine the brain regions involved in the task and whether they could be covered in a limited field of view at higher temporal resolution. An 
interesting possibility for future investigation would be to apply GCM to a task with relatively simple, possibly known, regional interactions, scanned multiple times with different degrees of spatial coverage and temporal resolution. This could potentially provide insight to the power of GCM to resolve interactions at different points in the trade-off between temporal and spatial resolution in fMRI.

It was also shown in the simulations in chapter 1 that the stochastic information loss in the low-pass filtering of the hemodynamics and the coarse temporal sampling of the MR scanner can result in bias in the inferences of individual Granger causality terms. It was argued that, to obtain unbiased statistics for GCMs, inference must be performed on difference maps (dGCMs) of the form Ref2Vox - Vox2Ref, which assign either a influence from reference region to a map-voxel (positive value), or an influence from a map-voxel to the reference region (negative value), but not both at the same time. This means that true bidirectional interactions between regions of equal strength and timedelay are lost. Only the dominant direction of influence is likely to be made visible. Thus GCM does not seem to be well suited for investigations of fast bi-directional feedback loops. This is likely to be a fundamental limit of the technique, owing to its fundament in BOLD fMRI. Despite the possible increases in resolution possible by restricting field of view, lowering spatial resolution, and innovations in measurement methods, the indirect access to neuronal activity through hemodynamic mechanisms limits the fundamental temporal resolution achievable. Although this fundamental lower limit might extend as far as a few hundreds of milliseconds (Formisano and Goebel, 2003), the degree of temporal smoothing introduced into the signal by the hemodynamics will likely make inference on the fastest and most symmetric bi-directional interactions impossible. As discussed below, EEG or MEG measurements, with their higher temporal resolution, could in some cases be of considerable added value in investigations of directed influence.

Because the GCM technique maps influences between regions considering them as a single interacting pair, no sound conclusions can be drawn about the 'directness' of these interactions, i.e. whether or not an influence could be mediated by a third region or structure. Assessing influences in the context of a larger network of involved regions requires estimation of a multivariate model that contains all important regions, e.g. an $\mathrm{N}$ dimensional autoregressive model. This would enable the computation of conditional GCMs based on conditional influence measures (Geweke, 1984), which partial out the influences of possibly confounding regions. The extension of GCM to this conditional form is a possible future extension that could enable more reliable inferences on mediating regions or sources of common input. A further extension could be that to nonlinear dynamic models, capable of capturing non-linear interactions between regions. An important case of a non-linear interaction is the modulation of the interaction between two regions by a third region. For instance, modulatory interactions have shown in an attention to motion task where the influence exerted by early visual areas on motion sensitive V5 area was found to be modulated by an area in the posterior parietal cortex, as detected by non-linear effective connectivity models (Buchel and Friston, 1997; Friston and Buchel, 2000; Harrison et al., 2003). However, the amount of parameters in a full multivariate Vector Autoregressive model with non-linear terms can become very large, requiring many data-points for its estimation. Furthermore, the possible distorting 
influence of the information loss in the hemodynamics and temporal sampling on more complicated non-linear and conditional influence terms will also need close investigation.

An interesting future direction for the investigation of effective connectivity in the brain with Granger causality, is the acquisition of both fMRI and EEG or MEG data for a task under investigation. The application of Granger causality to electrophysiological methods has shown to be successful as an approach to the investigation of large-scale neuronal interactions in the brain (Bernasconi and Konig, 1999; Brovelli et al., 2004; Cassidy and Brown, 2002; Chavez et al., 2003; Freiwald et al., 1999; Hesse et al., 2003; Kaminski et al., 2001). In fact, the high temporal resolution of electrophysiological methods like EEG and MEG seems to make it an ideal candidate for the application of Granger causality, since it is based on the temporal structure in the statistical dependency of signals. However, the spatial resolution of these methods is considerably lower than that of fMRI, and they are unable to record activity from deep brain sources. This motivated the development of GCM that uses both the high spatial resolution and the temporal information available in BOLD fMRI time-courses to map Granger causal influence over the whole brain at high spatial resolution. For fMRI, as discussed above, the fundamentally limited temporal resolution and temporal uncertainty caused by the intervening hemodynamic processes are the cause of likely limits in the application and interpretation of Granger causality in fMRI time-courses.

The complementary strengths and weaknesses of the hemodynamic/metabolic methods and the electrophysiological methods are expressed in their complementary possibilities for functional integration investigations. For instance, the canonical case of two neuronal populations in close proximity to each other in the brain (a few millimeters) interacting at a considerable delay could be detected by fMRI GCM, whereas the power of EEG/MEG to spatially resolve the signals of these populations will be limited. On the other hand, a second canonical case where two neuronal population located at some distance (a few centimeters) interact bi-directionally in a rapid fashion would potentially be detectable by EEG/MEG, whereas fMRI based effective connectivity methods will be much more limited in their power to resolve influences in this case. Conversely, EEG/MEG investigations of effective connectivity will likely fail to detect interactions similar to the first case, whereas fMRI based connectivity investigations will probably miss interactions of the second kind. Interactions of either kind are very likely to occur in the brain. Thus, in the strictest sense, each method individually is likely to provide a biased view on neural system interactions dictated by its fundamental temporal or spatial limits. The obvious path towards a solution would be to combine both methods in investigations of functional integration in the brain.

However, the mere recognition of a possible direction of improvement does not instantly move a scientific field along. Although the availability of both $\mathrm{fMRI}$ and EEG/MEG data for a task or cognitive process under investigation could in principle allow the investigation of directed cortical interactions with both high spatial and high temporal resolution, there are important practical and technical problems to overcome before this is feasible. First, besides their differences in spatial and temporal resolution of acquisition, fMRI and EEG/MEG are known to reflect subtly different aspect of neuronal activity. EEG/MEG mostly reflects the summed dipole moment generated by synaptic input at apical dendrites of large populations of pyramidal neurons of a specific coherent orientation when they signal in synchrony. The BOLD fMRI signal reflects a subtle 
combination of changes in capillary blood flow, blood volume and hemoglobin oxygenation, probably resulting more from synaptic activity and the generation of graded post-synaptic potentials than from axonal action potentials (Logothetis et al., 2001). Second, the experimental design and paradigms used with fMRI and EEG/MEG differ in subtle but important ways. Even in event-related experimental designs, used with both methods, the inter trial interval is kept long (allowing only few trials) in fMRI studies to keep event-related responses in the BOLD signal temporally separate (even in modern 'fast' event-related designs), whereas it is kept as short as possible (permitting many trials) in EEG/MEG to allow averaging to increase the signal to noise ratio. Thus, performing the exact same task while recording separately with both methods will take very subtle and flexible experimental design. More technical issues are to be overcome if an even more direct combination of both modalities is the goal, either by simultaneous acquisition of both EEG and FMRI in the MR scanner, or by co-localization of both fMRI and EEG signals through fMRI-constrained dipole modeling. Although simultaneous acquisition of both EEG and $\mathrm{PMRI}$ could provide greater conformity and congruence in the recorded signals, the quality of the individual signals decreases from the effect of the magnetic gradients on the EEG sensors, and, in turn, the disturbing effects of the magnetically non-neutral sensors on the MR signal. The localization of EEG or MEG activity to the cortical surface by regularized solutions to the inverse problem, possibly constrained by fMRI activation for the same task, potentially provides access to both signals at the same anatomical location. However, the precision and reliability of localization of EEG/MEG activity is still a topic of active research. Furthermore, in the context of functional integration studies, the interaction of two highly complex modeling processes, the spatial localization model and the temporal effective connectivity model, will require investigation and validation. Finally, one should be aware that certain types of interactions, specifically fast bidirectional interactions between neuronal structures in close spatial proximity, will remain difficult to detect even with a combination of the two acquisition methods.

\section{Diffusion tensor imaging and fiber tracking}

In this thesis high resolution diffusion tensor imaging of the human optic chiasm was performed ex vivo at ultra-high field to validate of the diffusion tensor model and streamline tractography algorithms. The complex micro-structure of the optic chiasm where complex fiber configurations are present, such as kissing, crossing, convergence and sharp curves, was well characterized at the acquired resolution. Stream-line tractography by a line propagation algorithm was used to investigate to which degree the known macroscopic connectivity through the optic chiasm could be reproduced. Overall, fibers crossing from optic nerve to contralateral optic tract, that should be the most numerous, were underrepresented, whereas fibers connecting VOls on the same side of the horizontal or vertical midline were heavily overrepresented. The overrepresentation of tracts connecting optic nerve with the ipsilateral optic tracts and connections between the two nerves or between the two tracts are mainly the result of problems of the tracking algorithm at the two regions of complex fiber architecture. The under-representation of fibers crossing from optic nerve to contralateral VOI, and the over-representation of ipsilateral nerve-to-tract connections got more severe with decreasing resolution. 
Moreover, the actual paths traced out in the down-sampled data-sets for the remaining crossing fiber were seen to be less veridical. These distorted paths seem to be caused by the distorted tensor fields resulting from partial volume averaging in the lower resolution datasets. Combination of high-resolution imaging with more classical ways of determining anatomical connectivity, such as tracer injections, seems the most suitable way of providing a gold standard for the validation tractography results. Because actively transported tracers will be unsuitable for post-mortem tracer studies, passively diffusing tracers will have to be used. In particular, injection of passively diffusing dyes, at discrete tissue sites with different axonal projections will be of great value for the validation of tractography results.

In this thesis DTI tractography was considered as a potential source of information on anatomical connectivity in the human brain to be used to inform and constrain models of effective connectivity. Ideally, after the set of regions and structures involved in a task of interest are identified (e.g. with the help of the GCM method), DTI tractography would provide the true anatomical model for the connections between these regions. However, in its current state diffusion MR based tractography does not seem to be able to live up to that ideal. Although promising first results have been obtained using DTI-based tractography, many investigators have come to the conclusion that its results cannot currently deliver the ground-truth true connectivity in the living human brain with large certainty. The validation work in this thesis is another body of support to that provisional conclusion, showing that both the amount of false positive results and false negative results of DTI tractography can be considerable. However, there is also much room and vision for improvement, that could conceivably lead to much more reliable results in the future. Four loosely separate strands of recent developments are likely to contribute to the improved accuracy of diffusion MR based tractography: 1) improved MR acquisition techniques and spatial resolution, 2) high angular resolution measurements and nongaussian diffusion models, 3) improved tractography algorithms, and 4) tractographybased parcellation of cortical and subcortical structures.

Many of the problems of representing complex fiber architectures, such as fiber crossings or high curvature, in a voxel-wise diffusion model result from partial volume averaging in relatively large voxels. The larger voxels are with respect to the whitematter anatomy of interest, the more separate population of differently oriented fibers will be averaged into single voxels, and the more difficult the accurate representation of the fiber architecture becomes, as also the work in this thesis has shown. Therefore, one obvious way of attacking these problems is to increase the acquired resolution of diffusion-weighted sequences while maintaining an adequate signal-to-noise ratio. Although by far the most straightforward way of increasing spatial resolution in MRI is to increase imaging times, the leverage to be gained from this in human brain imaging is limited by the short time a subject can be expected to lie inside an MR-scanner. The rapid developments in MR scanner hardware and acquisition techniques in recent years are capable of achieving spatial resolution increases. The move to increasingly higher static fields for human brain MRI is one such development. The base signal available for MR imaging is directly proportional both to the strength of the static magnetic field employed and to the acquired voxel size. The increased baseline signal-to-noise ratio (SNR) at higher fields allows an increase in spatial resolution. The DTI tractography study in this thesis was performed at $9.4 \mathrm{~T}$ with long imaging times giving a resolution of 
$156 \mu \mathrm{m}$ in-plane, which is far beyond the resolutions achievable with standard clinical scanners at $1.5 \mathrm{~T}$ or $3 \mathrm{~T}$ used in most cognitive neuroscience research, even if similar imaging times would be used. The availability of MR scanners capable of human brain imaging at $7 \mathrm{~T}$, currently under development at multiple sites, could potentially increase the acquired resolution of diffusion weighted data considerably beyond the current voxel dimensions not much smaller than $2 \times 2 \times 2 \mathrm{~mm}^{3}$ for clinically usable sequences at $1.5 \mathrm{~T}$ or 3T. New acquisition techniques, such as parallel imaging also have the potential to increase signal-to-noise ratio and spatial resolution, while reducing distortions (Jaermann et al., 2004).

Some of the obvious errors made by the DTI-based line propagation algorithm clearly arise from the inadequacy of the diffusion tensor model to represent complex fiber architecture, such as fiber crossings. Recently, alternative models, capable of representing non-gaussian diffusion profiles in voxels with complex fiber architectures have been proposed (Alexander et al., 2002; Assaf et al., 2004; Frank, 2002; Jansons and Alexander, 2003; Liu et al., 2004; Ozarslan and Mareci, 2003; Tuch et al., 2002; Tuch et al., 2003; Wedeen et al., 2005). The estimation of these models require high angular resolution diffusion imaging (HARDI) acquisitions, where the angular resolution of the diffusion direction sampling is greatly increased, from six directions, needed for estimation of the diffusion tensor, to tens or even hundreds of directions. The increased resolution of the angular sampling of the local diffusion within a voxel allows estimation of more complex diffusion models with more parameters, better able to characterize complex multi-peaked diffusion profiles. The more detailed characterization of diffusion within each voxel can be expected to improve the results of fiber tracking, particularly in problematic situations, such as fiber crossings. However, the increased directional sampling also increases the amount of diffusion-weighted measurements needed. Thus, the required imaging time rapidly increases linearly with the amount of sampled directions. Given that imaging time for a live human subject is limited, the increase in angular resolution will come at the cost of decreased SNR for each sampled direction and/or a decrease in spatial resolution. Furthermore, the absolute amount of diffusion weighting, quantified in the b-value, to be used is somewhat of an uncertain parameter. More diffusion weighting (higher b-values) will give more contrast between subtle differences in diffusion for different directions, potentially leading to 'sharper' estimated diffusion profiles for complex fiber architecture (Alexander et al., 2001; Frank, 2002). However, more diffusion weighting requires either longer or stronger diffusion gradients. Given that the gradient strength for human imaging must be kept limited to avoid peripheral nerve stimulation effects, high b-value diffusion weighting requires long diffusion gradients that increase echo times and as a consequence the SNR of the measurements. Investigation in the near future will be required to assess the relative merits of the many different non-gaussian diffusion models recently proposed and how they interact with the trade-offs in the acquisition process between spatial resolution, angular resolution, and signal-to-noise ratio.

Recent developments in tractography algorithms have aimed at removing two constraints of the line propagation algorithm: its use of only local information at the current tracking point, and its lack of providing any kind of reliability measure for a resulting streamline. First, some developments have taken place in tractography algorithms that minimize a more global fit of a stream-line to the discrete estimated 
tensor field through which it passes, for instance by estimating geodesics in the tensor field considered as a Riemannian manifold (Lenglet et al., 2004), or by using the Metropolis algorithm, a simulated annealing approach that iteratively maximizes the fit of the entire tract to the data (Tuch, 2002). Potentially, these algorithms are less prone to errors at critical regions when a wrong path through a critical region will decrease the fit to the surrounding data. Second, many recent studies (Behrens, Woolrich et al., 2003; Campbell et al., 2005; Hagmann et al., 2003; Jackowski et al., 2005; Koch et al., 2002; Parker and Alexander, 2003; Parker et al., 2003; Parker et al., 2002) have investigated so-called probabilistic tractography algorithms that provide for a given seedpoint not a single most likely terminal point of connection along with a joining tract (as the 'deterministic' line propagation algorithms do), but rather a 3-D map of connection probability. For each region in the brain, the resulting map will give a probabilistic index of connectivity (PICo) for the likelihood of the existence of an anatomical connection to the seed region, given the data. Such a characterization of connectivity is much more useful, in the light of the potential inaccuracies of general fiber tracking, than the misleading 'yes-or-no' answer provided by a deterministic line propagation algorithm. However, it should be noted that the use of a stochastic tractography algorithm per se does not necessarily result in more accurate fiber tracking. The evolution of the path of a randomly perturbed particle or a propagating front, used by these algorithms, is generally constrained by the same local information at the point of propagation (shapes of estimated tensors) and the same constraints (anisotropy thresholds, angle thresholds) as the standard deterministic line propagation algorithms are. Thus the same problems can arise, such as erroneous jumping into a different tract at sites of convergence or divergence, wrong interpretation of kissings or crossings of tracts, or failure to follow through a tract of high curvature. Consequently, the interpretation of probabilistic connectivity maps is potentially hindered by similar problems as the interpretation of deterministic stream-lines. False positives, regions with a high computed PICo that are not truly anatomically connected, and false negatives, areas with a low PICo that have a direct afferent or efferent axonal connection with the seed-region, are still likely to occur. A promising line of investigation will be that of combining the increased accuracy resulting from non-gaussian local diffusion models and globally fitted stream-lines within a probabilistic tractography algorithm, such that better accuracy of tractography is combined with a measure of reliability. However, regardless of the eventual accuracy attainable, it should be realized that certain fundamental indeterminacies are present in the diffusion profiles. The directional polarity of a tract (anterograde or retrograde) can not be determined from the diffusion-weighted signal. Thus, distinctions between afferent and efferent pathways can not be made on the basis on diffusion MR based tractography alone. Furthermore, certain complex configurations will always remain hard to resolve, since alternative possibilities give rise to (virtually) the same diffusion profiles, such as a kissing vs. crossing configuration. Validation of local diffusion models and tractography algorithms, for instance by application to post-mortem tissue samples combined with molecular tracer techniques, will be essential to assess the degree of accuracy that is eventually attainable.

Despite the inaccuracies of fiber tracking based on diffusion MR, the information present in the tractography based stochastic connection profile is considerable. Some recent investigations have shown that it can be useful to shift focus from single area-to- 
area connections to the distributed connection pattern of a seed region to the rest of the brain. Anatomically and functionally distinct cortical regions or subcortical structures have different connection patterns to the rest of the brain. Conversely, if information is available on the connection pattern of an unknown neural structure, that information could be used to classify that structure as being anatomically and functionally distinct from other structures with a different connection profile. This idea was exploited in recent investigations partitioning and classifying the thalamus into its different nuclei (Behrens, Johansen-Berg et al., 2003), and the medial prefrontal motor cortex into the supplementary and pre-supplementary motor area (Johansen-Berg et al., 2004), based on stochastic tractography information. All voxels in a region of interest (the thalamus or medial prefrontal cortex) in the MR data volume are used as a seed point for a stochastic tractography algorithm, providing a probabilistic connectivity map for each voxel. Voxels with a similar connection profile are then classified as belonging to the same structure, for instance by a clustering algorithm. Even though each of the connectivity maps is likely to contain errors and inaccuracies, the global information in the similarity structure of these maps in enough to reliably classify neural structures in the MR dataset. Just like in the case of the single stream-line discussed above, taking a more global view on the information in diffusion MR data-sets seems to lead to more accurate results. The connectivity based parcellation approach could potentially be very useful for effective connectivity studies, despite the fact that it does not provide information about the exact connectivity profile of each classified region. First, a reliable classification of cortical and subcortical areas beyond brodmann areas or generic brain template coordinates is extremely useful for the interpretation of functional activation in general. Particularly when this classification is performed individually in each subject, idiosyncrasies and similarities in the exact functional location of activation can be interpreted and used. Second, although no definitive information about the anatomical connections of the classified areas is given, the stochastic connectivity maps provide strong suggestions for the possible connections with other structures. Especially when this tentative information is combined with information available in anatomical atlases, tracer studies, and, particularly, human brain atlases based on post-mortem histology (Burgel et al., 2006; Eickhoff et al., 2006).

\section{Outlook}

The investigation of functional integration in the brain is a crucial part of modern cognitive neuroscience. The brain is a highly complex structure subdivided at different levels into many semi-independent modules. Although parallel and distributed processing is a key strategy in the neural processes that underlie perception, cognition, and behavior, many large scale modules in the brain are specialized to perform distinct functions or computations. The full complexity of cognitive processing arises in the interactions of these semi-modular structures. The complementary investigation of both functional specialization and functional integration will remain an important paradigm in cognitive neuroscience studies.

Most studies of functional integration to date have applied methods for the detection of functional connectivity or effective connectivity to either EEG/MEG or FMRI data. It 
was argued above that combined fMRI and EEG/MEG studies of a cognitive task or process of interest could extend the possibilities for investigation of large scale neural system interactions. This combination need not be explicit, in the sense that EEG data is recorded simultaneously with $f M R I$ in the MR-scanner or that EEG/MEG data is computationally localized to the cortex and analyzed in the same reference space as the fMRI data, although these developments might provide benefits. Even without explicit combination of the data the availability of both fMRI and EEG/MEG data for the same task has the potential to give a window onto both temporally fast, bidirectional interactions and interactions between structures in close spatial proximity, combining the strengths of both methods. Important initial investigations could aim at acquisition of both EEG or MEG data and fMRI data for a simple, relatively well understood task. As a first step, Granger causality could be applied to both types of datasets to qualitatively compare the results. One could validate whether relatively slow interactions between spatially separate cortical regions are reflected in the Granger causal influences found both in the $\mathrm{FMRI}$ and EEG or MEG dataset. The possibility of frequency decompositions of the EEG or MEG signals and of the influence measures based on those signals, allow the investigation of the frequency bands in the electrophysiological signals that contribute most to the Granger causal influences in the fMRI signal. Furthermore, there is much useful information in the complementary cases where influence is found in only one of the two modalities. Is influence uniquely found in the electrophysiological data indeed of a fast or bidirectional nature? Is Granger causality found only in the fMRI datasets between regions in close spatial proximity or influence involving subcortical structures? Finally, the fMRI data could be acquired at a number of different TRs, to investigate the effect of the acquired temporal resolution and the trade-off between spatial and temporal resolution on fMRI Granger causality and its comparison to EEG/MEG Granger causality.

This thesis has investigated the potential of DTI based fiber tracking to provide information about individual anatomical connectivity to complement and constrain effective connectivity models. In its current state DTI based tractography cannot reliably track arbitrary individual fiber bundles with high certainty, as shown in the validation study in chapter 3 . However, as argued above, there is much room for improvement in tractography results, and a more global view on tracking results could provide very useful information for studies of functional integration. Future studies can aim at combining connectivity based parcellation of brain structures with effective connectivity measures. An interesting study would be one in which parcellation of a specific cortical region (e.g. the medial prefrontal cortex), is combined with fMRI GCM for task known to involve that cortical region. A first qualitative comparison of the results of the two methods would be highly informative, for instance in verifying whether sub-regions highlighted in the GCMs are specific to the regions demarcated by the parcellation. Furthermore, for a given subregion one could investigate the overlap between its sources and targets of Granger causal influence on the one hand, and its anatomical connections suggested by the tractography on the other. Such combined exploratory studies of effective connectivity and anatomical connectivity can be of great use in early stages of the investigation of the functional networks underlying a task of interest. Eventually such studies can provide enough information to formulate explicit hypotheses in the context of an inferred prespecified structural model that could be testing with existing effective connectivity 
models, such as covariance structural equation modeling (McIntosh and Gonzalez-Lima, 1994) or dynamic causal modeling (Friston et al., 2003). Of course, ideally these hypotheses should be tested on data independent of that on which the initial exploration was done, to maintain rigorous statistical inference and avoid capitalization on chance.

Finally, it should be noted that even the specification of a fully anatomically correct network model for a given task, together with equations specifying the causal flow of activity through it, does not embody our final and ultimate understanding of the modeled task or process. Effective connectivity models to date, GCM included, have largely modeled each of the functional regions in a network as 'black boxes' characterized by a few (often one) state variables describing aspects of their activity at a given time. Such models are of great importance in a paradigm of 'top-down' modeling of neural processing and cognitive function. In this top-down modeling paradigm, characteristic of effective connectivity studies, the functional role played by a region and the interaction between regions or its modulation, is inferred from recorded activity data. Theoretical inference moves from the data down to the modules that generated it. This paradigm has provided many important insights into the functional working of the brain. However, by its very nature it is unable to provide more than circumstantial information about what happens inside each of the interacting functional modules. The black boxes remain opaque to our understanding of their internal workings. In the future, the top-down effective connectivity paradigm should complemented by a 'bottom-up' paradigm that explicitly models the function of individual regions, presumably in the form of a neural network model (Horwitz, 2004; Horwitz and Tagamets, 1999; Horwitz et al., 2005). Such large scale neuronal network models have been constructed for e.g. a visual delayed match-to-sample task (Tagamets and Horwitz, 1998), visual attention mechanisms (Corchs and Deco, 2002), and the auditory continuity illusion (Husain et al., 2005). Large scale neural network models contain explicit hypotheses about the functions of the individual modules and how these functions are implemented within the modules as neuronal computations. The crucial aspect of such models is that they were constructed to be able to perform the task under investigation. The propagation of activity through such models supports the performance of its function and, if the model is correct, should parallel the causal flow of activity observed in the brain of a subject performing the task. Thus, a large scale neural network model can generate 'bottom-up' hypotheses about the expected temporal flow of activity and interaction between brain regions than can be tested on actual data, and be used to favor one theoretical model over the other. In other words, theoretical inference moves from the model to the data in this paradigm. The active use and integration of these two complementary modeling approaches can further extend the possibilities to investigate functional integration in the brain and further our understanding of the neuronal substrates of perception, cognition, and action. 


\section{References}

Alexander AL, Hasan KM, Lazar M, Tsuruda JS, Parker DL (2001) Analysis of partial volume effects in diffusion-tensor MRI. Magn Reson Med 45: 770-780.

Alexander DC, Barker GJ, Arridge SR (2002) Detection and modeling of non-Gaussian apparent diffusion coefficient profiles in human brain data. Magn Reson Med 48: 331-340.

Assaf Y, Freidlin RZ, Rohde GK, Basser PJ (2004) New modeling and experimental framework to characterize hindered and restricted water diffusion in brain white matter. Magn Reson Med 52: 965-978.

Behrens TE, Johansen-Berg H, Woolrich MW, Smith SM, Wheeler-Kingshott CA, Boulby PA, Barker GJ, Sillery EL, Sheehan K, Ciccarelli O, Thompson AJ, Brady JM, Matthews PM (2003) Non-invasive mapping of connections between human thalamus and cortex using diffusion imaging. Nat Neurosci 6: 750-757.

Behrens TE, Woolrich MW, Jenkinson M, Johansen-Berg H, Nunes RG, Clare S, Matthews PM, Brady JM, Smith SM (2003) Characterization and propagation of uncertainty in diffusionweighted MR imaging. Magn Reson Med 50: 1077-1088.

Bernasconi C, Konig P (1999) On the directionality of cortical interactions studied by structural analysis of electrophysiological recordings. Biol Cybern 81: 199-210.

Brovelli A, Ding M, Ledberg A, Chen Y, Nakamura R, Bressler SL (2004) Beta oscillations in a largescale sensorimotor cortical network: Directional influences revealed by Granger causality. Proc Natl Acad Sci U S A 101: 9849-9854.

Buchel C, Friston KJ (1997) Modulation of connectivity in visual pathways by attention: cortical interactions evaluated with structural equation modelling and fMRI. Cereb Cortex 7: 768778.

Burgel U, Amunts K, Hoemke L, Mohlberg H, Gilsbach JM, Zilles K (2006) White matter fiber tracts of the human brain: Three-dimensional mapping at microscopic resolution, topography and intersubject variability. Neuroimage 29: 1092-1105.

Campbell JS, Siddiqi K, Rymar VV, Sadikot AF, Pike GB (2005) Flow-based fiber tracking with diffusion tensor and q-ball data: Validation and comparison to principal diffusion direction techniques. Neuroimage.

Cassidy MJ, Brown P (2002) Hidden Markov based autoregressive analysis of stationary and nonstationary electrophysiological signals for functional coupling studies. J Neurosci Methods 116: 35-53.

Chavez M, Martinerie J, Le Van Quyen M (2003) Statistical assessment of nonlinear causality: application to epileptic EEG signals. J Neurosci Methods 124: 113-128.

Corchs S, Deco G (2002) Large-scale neural model for visual attention: integration of experimental single-cell and fMRI data. Cereb Cortex 12: 339-348.

Eickhoff SB, Heim S, Zilles K, Amunts K (2006) Testing anatomically specified hypotheses in functional imaging using cytoarchitectonic maps. Neuroimage.

Formisano E, Goebel R (2003) Tracking cognitive processes with functional MRI mental chronometry. Curr Opin Neurobiol 13: 174-181.

Frank LR (2002) Characterization of anisotropy in high angular resolution diffusion-weighted MRI. Magn Reson Med 47: 1083-1099. 
Freiwald WA, Valdes P, Bosch J, Biscay R, Jimenez JC, Rodriguez LM, Rodriguez V, Kreiter AK, Singer W (1999) Testing non-linearity and directedness of interactions between neural groups in the macaque inferotemporal cortex. J Neurosci Methods 94: 105-119.

Friston KJ, Buchel C (2000) Attentional modulation of effective connectivity from V2 to V5/MT in humans. Proc Natl Acad Sci U S A 97: 7591-7596.

Friston KJ, Harrison L, Penny W (2003) Dynamic causal modelling. Neuroimage 19: 1273-1302.

Geweke JF (1984) Measures of Conditional Linear Dependence and Feedback Between Time Series. Journal of the American Statistical Association 79: 907-915.

Hagmann P, Thiran JP, Jonasson L, Vandergheynst P, Clarke S, Maeder P, Meuli R (2003) DTI mapping of human brain connectivity: statistical fibre tracking and virtual dissection. Neuroimage 19: 545-554.

Harrison L, Penny WD, Friston K (2003) Multivariate autoregressive modeling of fMRI time series. Neuroimage 19: 1477-1491.

Hesse W, Moller E, Arnold M, Schack B (2003) The use of time-variant EEG Granger causality for inspecting directed interdependencies of neural assemblies. J Neurosci Methods 124: $27-$ 44.

Horwitz B (2004) Relating fMRI and PET signals to neural activity by means of large-scale neural models. Neuroinformatics 2: 251-266.

Horwitz B, Tagamets MA (1999) Predicting human functional maps with neural net modeling. Hum Brain Mapp 8: 137-142.

Horwitz B, Warner B, Fitzer J, Tagamets MA, Husain FT, Long TW (2005) Investigating the neural basis for functional and effective connectivity. Application to fMRI. Philos Trans R Soc Lond B Biol Sci 360: 1093-1108.

Husain FT, Lozito TP, Ulloa A, Horwitz B (2005) Investigating the neural basis of the auditory continuity illusion. J Cogn Neurosci 17: 1275-1292.

Jackowski M, Kao CY, Qiu M, Constable RT, Staib LH (2005) White matter tractography by anisotropic wavefront evolution and diffusion tensor imaging. Med Image Anal 9: 427-440.

Jaermann T, Crelier G, Pruessmann KP, Golay X, Netsch T, van Muiswinkel AM, Mori S, van Zijl PC, Valavanis A, Kollias S, Boesiger P (2004) SENSE-DTI at 3 T. Magn Reson Med 51: 230-236.

Jansons KM, Alexander DC (2003) Persistent Angular Structure: new insights from diffusion MRI data. Dummy version. Inf Process Med Imaging 18: 672-683.

Johansen-Berg H, Behrens TE, Robson MD, Drobnjak I, Rushworth MF, Brady JM, Smith SM, Higham DJ, Matthews PM (2004) Changes in connectivity profiles define functionally distinct regions in human medial frontal cortex. Proc Natl Acad Sci U S A 101: 1333513340.

Kaminski M, Ding M, Truccolo WA, Bressler SL (2001) Evaluating causal relations in neural systems: granger causality, directed transfer function and statistical assessment of significance. Biol Cybern 85: 145-157.

Koch MA, Norris DG, Hund-Georgiadis M (2002) An investigation of functional and anatomical connectivity using magnetic resonance imaging. Neuroimage 16: 241-250.

Lenglet C, Deriche R, Faugeras O (2004) Inferring white matter geometry from diffusion tensor MRI: Application to connectivity mapping. In: (Pajdla $\mathrm{T}$, Matas $\mathrm{J}$, eds.) 8th European Conference on Computer Vision. Prague, Czech Republic: Springer--Verlag.

Liu C, Bammer R, Acar B, Moseley ME (2004) Characterizing non-Gaussian diffusion by using generalized diffusion tensors. Magn Reson Med 51: 924-937. 
Logothetis NK, Pauls J, Augath M, Trinath T, Oeltermann A (2001) Neurophysiological investigation of the basis of the fMRI signal. Nature 412: 150-157.

McIntosh AR, Gonzalez-Lima F (1994) Structural Equation Modeling and Its Application to Network Analysis in Functional Brain Imaging. Human Brain Mapping 2: 2-22.

Ozarslan E, Mareci TH (2003) Generalized diffusion tensor imaging and analytical relationships between diffusion tensor imaging and high angular resolution diffusion imaging. Magn Reson Med 50: 955-965.

Parker GJ, Alexander DC (2003) Probabilistic Monte Carlo based mapping of cerebral connections utilising whole-brain crossing fibre information. Inf Process Med Imaging 18: 684-695.

Parker GJ, Haroon HA, Wheeler-Kingshott CA (2003) A framework for a streamline-based probabilistic index of connectivity (PICo) using a structural interpretation of MRI diffusion measurements. J Magn Reson Imaging 18: 242-254.

Parker GJ, Stephan KE, Barker GJ, Rowe JB, MacManus DG, Wheeler-Kingshott CA, Ciccarelli O, Passingham RE, Spinks RL, Lemon RN, Turner R (2002) Initial demonstration of in vivo tracing of axonal projections in the macaque brain and comparison with the human brain using diffusion tensor imaging and fast marching tractography. Neuroimage 15: 797-809.

Tagamets MA, Horwitz B (1998) Integrating electrophysiological and anatomical experimental data to create a large-scale model that simulates a delayed match-to-sample human brain imaging study. Cereb Cortex 8: 310-320.

Tuch DS (2002) Diffusion MRI of Complex Tissue Structure. In: Division of Health Sciences and Technology. Boston: MASSACHUSETTS INSTITUTE OF TECHNOLOGY.

Tuch DS, Reese TG, Wiegell MR, Makris N, Belliveau JW, Wedeen VJ (2002) High angular resolution diffusion imaging reveals intravoxel white matter fiber heterogeneity. Magn Reson Med 48: 577-582.

Tuch DS, Reese TG, Wiegell MR, Wedeen VJ (2003) Diffusion MRI of complex neural architecture. Neuron 40: 885-895.

Wedeen VJ, Hagmann P, Tseng WY, Reese TG, Weisskoff RM (2005) Mapping complex tissue architecture with diffusion spectrum magnetic resonance imaging. Magn Reson Med 54: 1377-1386. 
114 
Summary

115 
In the past few decades, the endeavor of the field of cognitive neuroscience has been to unravel the intricate neuronal processes in the brain that underlie perception, cognition and behavior. Numerous investigations using neuroimaging methods have led to the localization of neural activity tied to perceptual, cognitive, language and motor processes. This functional localization has been achieved both in time (primarily through the use of electrophysiological methods: Electro-Encephalography, EEG, and MagnetoEncephalography, MEG) and in space (primarily through the use of hemodynamic/metabolic methods: Positron Emission Tomography, PET, and functional Magnetic Resonance Imaging, fMRI). However, each neuronal population in the brain performs its tasks in continuous interaction with other neural structures. An important part of what a neural structure does is determined not only by its own local processing, but also by how it interacts with other systems. This is the functional integration view on brain function. The interaction of spatially separated neuronal systems requires connectivity. In the context of large-scale interaction between separate brain systems, three kinds of connectivity can be operationally distinguished: anatomical connectivity (a direct axonal connection between two structures), functional connectivity (a correlation between measures of activity of two structures), and effective connectivity (the influence that one neuronal structure exerts over another). Although conceptually distinct, there are important practical relations between the three types of connectivity. For instance, direct effective connectivity between two systems can not exist without anatomical connectivity. Furthermore, the existence of effective connectivity (a directed influence) from one structure to another usually also implies observable functional connectivity (correlation) between the two.

This thesis investigates functional integration in the brain by studying two techniques, one for measuring effective connectivity: Granger causality mapping, and one to identify anatomical connectivity: diffusion tensor imaging. Both techniques are considered as complements to other fMRI effective connectivity methods, already in active use within the field. These existing methods presuppose a structural model of regions involved in a cognitive task and the relevant anatomical connections that exist between these regions. fMRI Granger causality mapping is proposed as a data-driven way to explore regions in the brain that interact with a selected reference region as measured from their functional activity. Diffusion tensor imaging is investigated as a basis to identify anatomical connections between cortical and subcortical regions.

In chapter 1, Granger causality mapping (GCM) is proposed as an approach to explore effective connectivity between neuronal populations in fMRI data. GCM does not rely on a priori specification of a model that contains pre-selected regions and connections between these regions. This distinguishes it from other fMRI effective connectivity approaches that aim at testing or contrasting specific hypotheses about neuronal interactions. Instead, GCM relies on the concept of Granger causality to define the existence and direction of influence from information in the data. Temporal order information is exploited to compute Granger causality maps that identify voxels that are sources or targets of directed influence for a selected region-of-interest. The method is investigated by simulations, and by application to fMRI data of a complex visuomotor task. The exploratory approach of mapping influences between a region of interest and the rest of the brain, forms a useful complement to existing models of effective connectivity. 
The ability to flexibly change behavior according to context is an important human cognitive capacity. For instance, when driving a car, different actions are required when approaching a red or green traffic light. This capacity has been investigated in cognitive psychology and neuroimaging investigations with the 'task switching' paradigm. In this paradigm, a decision or response task is performed by the subject according to certain rules which change regularly. In chapter 2 , the Granger causality mapping technique is applied in the investigation of brain region interactions in task switching. To this end, the GCM technique is generalized to compute Granger causality maps for a group of subjects (random effects (RFX) inference). The group GCM technique is used to identify the unknown interactions between brain regions in an fMRI study of switching visuomotor association tasks. RFX GCM analysis showed a prefrontal network, containing dorsal premotor cortex (PMd) and the pre-supplementary motor areas (SMA/preSMA), to be involved in the selection of responses. A strong input to the left dorsal premotor cortex (PMd) was seen from the pre-supplementary motor areas (SMA/preSMA) at the selection of responses when conflict between competing responses has to be resolved. A fronto-parietal network, containing the superior parietal lobule (SPL), ventro-lateral prefrontal cortex (VLPFC), and the inferior parietal lobule (IPL), was involved in controlling sets of stimulus-response associations. The left SPL receives input from left VLPFC, underlying high-level control by superordinate level representations of taskssets, and from the IPL, reflecting stimulus-response reconfiguration processes at switches.

In chapter 3, high resolution diffusion tensor imaging of the human optic chiasm is performed ex vivo at ultra-high field. The optic chiasm is a challenge for diffusion tensor models and fiber tracking methods, because of its complex fiber micro-structure. At the same time, because the general large-scale connectivity of the optic chiasm is well known it is an ideal candidate for validation of Diffusion Tensor Imaging fiber tracking approaches in resolving inter-regional anatomical connectivity. Estimated diffusion tensors at this high resolution are shown to be able to capture complex fiber configurations such as sharp curves, and convergence and divergence of tracts, but are unable to resolve directions at sites of crossing fibers. Despite the complex microstructure of the fiber paths through the optic chiasm, all known connections are tracked by a line propagation fiber tracking algorithm. However, fibers crossing from the optic nerve to contralateral tract are heavily underrepresented, whereas ipsilateral nerveto-tract connections, as well as tract-to-tract connections are overrepresented. Moreover, erroneous nerve-to-nerve connections occur. The effects of spatial resolution, and the partial volume averaging of complex fiber architecture, on the performance of these methods is investigated. Errors made by the fiber tracking algorithm at high resolution are shown to increase at lower resolutions closer to those used in vivo. This study shows that post-mortem investigation of fixed tissue samples with DTI at high field strengths is important in the validation of diffusion models based on magnetic resonance imaging and fiber tracking algorithms.

In the general discussion, Granger causality mapping and diffusion tensor imaging are discussed in the context of effective connectivity studies. The concrete merits and problems of each technique are discussed, and recommendations for possible improvements and future extensions are made. Four considerations in the application and interpretation of GCM are discussed: 1) The effect of intervening hemodynamics in 
fMRI and how to avoid its confounding influence on Granger causality inferences, 2) the effect of the sparse temporal sampling of fMRI with respect to the activity of neuronal processes, 3) the problem of false causality that arises when certain structures relevant to a task are not considered, and 4) the problematic case of bidirectional interactions between neural populations for fMRI based Granger causality. Combined fMRI and EEG/MEG studies of a cognitive task or process of interest could extend the possibilities for investigation of large scale neural system interactions, and could improve the handling of these issues.

In its current state DTI based fiber tracking cannot reliably track arbitrary individual fiber bundles with high certainty, as shown in the validation study in chapter 3 . However, there is much opportunity for improvement in fiber tracking techniques, and a more global view on tracking results could provide very useful information for studies of functional integration in the brain. Future studies can aim at combining connectivity based classification of brain structures with effective connectivity measures. The active use and integration of $\mathrm{FMRI}$ and EEG/MEG based effective connectivity measures and DTI based fiber tracking can vastly extend the possibilities to investigate functional integration in the brain, and further our understanding of the neuronal substrates of perception, cognition, and action. 
Samenvatting 
In de laatste tientallen jaren is het doorlopende doel van de Cognitieve Neurowetenschappen geweest om de ingewikkelde neurale processen in het brein te ontrafelen die ten grondslag liggen aan waarneming, denkprocessen, en gedrag. Vele onderzoeken, die gebruik maken van neuroimaging methoden, hebben geleid tot de lokalisatie van neurale activiteit verbonden aan perceptie, cognitie, taal, en beweging. Deze functionele lokalisatie is bereikt zowel in de tijd (vooral door het gebruik van electro-physiologische methoden: Electro-Encephalography, EEG, en MagnetoEncephalography, MEG), als in ruimte (vooral door het gebruik van hemodynamisch/metabolische methoden: Positron Emission Tomography, PET, en functional Magnetic Resonance Imaging, fMRI). Echter, de lokalisatie van gespecialiseerde neurale structuren is, alhoewel zeer belangrijk, niet het hele verhaal. Elke neurale populatie in het brein voert zijn taken uit in continue interactie met andere neurale structuren. Een belangrijk deel van wat een neurale structuur doet wordt niet alleen bepaald door de lokale verwerkingsprocessen die erin plaatsvinden, maar ook door hoe deze structuur samenwerkt met andere systemen in het brein. Dit is het functionele integratie perspectief op de werking van het brein. Deze interactie van neurale systemen in verschillende delen van het brein vereist connectiviteit tussen deze systemen. In deze context kunnen we een operationeel onderscheid maken tussen drie soorten van connectiviteit: anatomische connectiviteit (een directe axonale verbinding tussen twee structuren), functionele connectiviteit (een correlatie tussen maten van activiteit van twee structuren), en effectieve connectiviteit (de invloed die de ene structuur heeft op de andere). Alhoewel ze conceptueel van elkaar verschillen, zijn er belangrijke praktische relaties tussen de drie soorten connectiviteit. Bijvoorbeeld, directe effectieve connectiviteit (invloed van de een naar de ander) tussen twee systemen kan niet bestaan zonder anatomische connectiviteit. En effectieve connectiviteit impliceert meestal ook een waarneembare functionele connectiviteit (correlatie). De drie soorten connectiviteit zijn de operationele concepten waarmee functionele integratie in het brein in de praktijk onderzocht wordt.

Dit proefschrift onderzoekt functionele integratie in het brein, door de studie van twee op MRI gebaseerde technieken. De eerste techniek, voor het meten van effectieve connectiviteit is Granger causality mapping. Granger causality mapping wordt voorgesteld als een door fMRI data gestuurde manier om corticale gebieden te verkennen die interacteren met een geselecteerd referentie gebied. De tweede techniek, voor de identificatie van anatomische connecties is diffusion tensor imaging. Diffusion tensor imaging en fiber tracking worden onderzocht als een benadering tot het identificeren van anatomische connecties tussen corticale en subcorticale gebieden in het levende brein. Beide technieken vullen bestaande methoden voor effectieve connectiviteit in fMRI data aan. De bestaande methoden vereisen aannamen in de vorm van een model van de corticale gebieden die betrokken zijn in een cognitieve taak en alle relevante anatomische verbindingen tussen die gebieden.

In hoofdstuk 1 wordt Granger causality mapping (GCM) voorgesteld als een benadering tot het verkennen van effectieve connectiviteit tussen neurale populaties met fMRI. GCM is niet afhankelijk van een vooraf gespecificeerd model dat een selectie van corticale gebieden bevat, samen met de aangenomen verbindingen tussen deze gebieden. Hierin verschilt GCM van andere benaderingen tot het meten van effectieve connectiviteit in fMRI, die zich richten op het testen of contrasteren van specifieke 
hypothesen over neurale interacties. GCM baseert zich op het concept van Granger causaliteit om het bestaan en de richting van causale invloed te detecteren uit fMRI data. Granger causaliteit behelst het gebruik van informatie over de volgorde van signaal variaties in de tijd om zgn. Granger causality maps te berekenen. Deze Granger causality maps identificeren corticale gebieden en subcorticale strukturen die bronnen of doelen zijn van gerichte causale invloed voor een geselecteerd gebied van interesse. De methode wordt onderzocht met behulp van simulaties, en door de toepassing ervan op fMRI data, gemeten voor een visuele stimulus-respons associatie taak. De verkennende benadering tot het in kaart brengen van causale invloeden tussen een gebied van interesse en de rest van het brein vormt een zeer nuttig aanvulling tot bestaande methoden voor het meten van effectieve connectiviteit.

Het vermogen om gedrag flexibel te veranderen naar aanleiding van de omgeving en context is een belangrijke menselijke capaciteit. Bijvoorbeeld, wanneer je auto rijdt, zijn er verschillende handelingen vereist wanneer je een groen of rood stoplicht nadert. Dit vermogen is in de cognitieve psychologie en cognitieve neurowetenschappen onderzocht met zgn. task switching experimenten. In dit soort experimenten, moet de proefpersoon een beslissing maken of een response geven bepaald door taakdefiniërende regels, die regelmatig veranderen. In hoofdstuk 2, wordt de Granger causality mapping techniek toegepast om de interacties in het brein bij task switching te onderzoeken. Voor dit onderzoek wordt de GCM techniek uitgebreid om Granger causality maps te berekenen voor een groep van proefpersonen (Random effects (RFX) GCM). Groep-gebaseerde GCM identificeerde een prefrontaal netwerk, dat de dorsale premotor cortex en pre-supplementaire motor cortex bevat, en dat betrokken is in de selectie van responsen. De linker PMd ontvangt input van de SMA/preSMA bij de selectie van responsen vooral wanneer er meerdere conflicterende responsen zijn. Een frontaal-parietaal netwerk, met daarin de superior parietal lobule (SPL), ventro-laterale prefrontale cortex (vLPFC), en de inferior parietal lobule (IPL), is betrokken bij het controleren van verzamelingen stimulus-response associaties. De linker SPL ontvangt input van de linker VLPFC, die de controle behelst door hogere orde representaties van taak-regels, en van de linker IPL, die betrokken is bij reconfiguratie processen bij het omschakelen van de taak-regels. Uit deze resultaten blijkt dat GCM een geschikte methode is om toepassing-gerichte vragen te beantwoorden over onbekende interacties tussen structuren in het brein.

Hoofdstuk 3 beschrijft een post-mortem diffusion tensor imaging (DTI) studie van het menselijke optische chiasma op hoge spatiele resolutie. Het optisch chiasma is een uitdaging voor diffusie tensor modellen en fiber tracking methoden, door de complexiteit van de zenuw-projecties (banen van axonen) op zeer kleine schaal. Tegelijkertijd, zijn de algemene verbindingen door het optisch chiasma op grote schaal goed bekend. Daarom deze structuur een zeer geschikt studie object voor de validatie van DTI en fiber tracking als technieken om anatomische connectiviteit te identificeren. De uit de data geschatte diffusie tensoren bleken bij de hoge gemeten resolutie goed in staat complexe zenuwbaan configuraties te karakteriseren, zoals sterk gebogen banen en de convergentie en divergentie van verschillende projecties. De diffusie tensor modellen bleken echter niet in staat de richtingen van axon-vezels op de plek van een kruising te identificeren. Ondanks de complexe structuur van axon-banen door het optisch chiasma, werden alle bekende connecties geïdentificeerd door het fiber tracking algoritme. Het aantal axonen 
dat kruist van de optic nerve naar de contra-laterale optic tract is echter aanzienlijk onder-gerepresenteerd. Tegelijkertijd wordt het aantal axonen dat aan dezelfde kant van het brein blijft aanzienlijk over-gerepresenteerd in de resultaten. Verder laat het fiber tracking algoritme foutieve connecties tussen de twee ogen zien.

De effecten van de spatiele resolutie van de data op de prestatie van DTI en fiber tracking wordt onderzocht. De fouten die het fiber tracking algoritme maakt op hoge spatiele resolutie worden groter naarmate de spatiele resolutie afneemt naar de resolutie die wordt gebruikt bij in vivo studies. Dit onderzoek laat het belang zien van post-mortem studies van gefixeerd weefsel met DTI op hoge veldsterkten voor de validatie van DTI en fiber tracking algoritmen.

In de algemene discussie, worden Granger causality mapping en diffusion tensor imaging besproken in de context van studies die zich richten op effectieve connectiviteit. De voor- en nadelen van elke techniek worden besproken, en aanbevelingen voor mogelijke verbeteringen en uitbreidingen worden gedaan. Vier punten in de toepassing en de interpretatie van GCM worden besproken: 1) Het effect van de zgn. hemodynamische mechanismen, waarop fMRI gebaseerd is, en hoe de verstorende invloed hiervan op de Granger causality maat is te vermijden, 2) Het effect van de relatief spaarzame bemonstering van fMRI data in de tijd vergeleken met de activiteit van neurale processen, 3) Het probleem van valse causaliteit die ontstaat als bepaalde structuren die relevant zijn voor een taak niet meegenomen worden in de analyse, en 4) Het problematische geval van bi-directionele interacties tussen neurale populaties voor Granger causaliteit gebaseerd op fMRI. Gecombineerde fMRI en EEG/MEG studies van een cognitieve taak of proces zouden de mogelijkheden om interacties tussen neurale systemen in het brein kunnen uitbreiden, en de aanpak van bovenstaande problemen kunnen verbeteren.

Met de huidige stand van zaken kunnen DTI en fiber tracking geen individuele bundels van axonen identificeren met een hoge graad van zekerheid, zoals de validatie studie in hoofdstuk 3 laat zien. Desondanks zijn er veel mogelijkheden voor verbetering in fiber tracking technieken. Daarbij kan een meer globaal perspectief op de resultaten van fiber tracking algoritmen zeer bruikbare informatie opleveren voor studies van functionele integratie in het brein. Toekomstig onderzoek zou zich moeten richten op het combineren van technieken voor de identificatie van effectieve connectiviteit met de classificatie van gebieden in het brein op basis van anatomische connectiviteit verkregen uit fiber tracking studies. De integratie van technieken voor de identificatie van effectieve connectiviteit gebaseerd op fMRI en EEG/MEG en op diffusie gebaseerde fiber tracking kan nieuwe mogelijkheden openen om functionele integratie in het brein te onderzoeken. Dit zal op den duur ons begrip vergroten van het neurale substraat van perceptie, cognitie, en actie. 


\section{Dankwoord}

Dankwoorden zijn geen wetenschap. Een dankwoord is altijd goed, onfalsifieerbaar, niet in een paradigma te vatten. Een dankwoord is wel een ongelofelijk beknopte samenvatting, een tot stroop gecondenseerd surrogaat voor iets dat eigelijk met veel meer woorden gezegd zou moeten worden. Maar conventie dicteert dat een proefschrift nu eenmaal circa 120 pagina's droge wetenschap moet bevatten en misschien 2 met dingen die nog belangrijker zijn. Hier zijn die van mij.

Rainer and Elia, my jedi-masters, you have taught me much of what I know in science: the finer aspects of performing experiments, analysing data, keeping an open view, writing things down. Most of all, perhaps, you have taught me that science cannot be perfect, and that it cannot, and should not, be done alone. Rainer, your legendary enthousiasm has taught me to also look at possibilities, not only at problems. Moreover, our shared inerests in computer programming and science-fiction have made of our conversations (not to mention our movie sessions) a pleasurable mixture of science and other fun stuff. Elia, our discussions, acompanied by Italian temperament, arm-waving, and walking around the room, have shown me how to keep looking at matters critically and to be driven by data (even without ICA...). I feel honored and grateful to have received my tuition from you two. There are still some things left I have to learn from you, and perhaps I can grow wise enough to return the favor sometimes.

I feel fortunate with the room-mates I have had at the Psychology department, only two of them, but the best I could wish for. Niko, you have showed me that there is always a different way of doing things and that even in the soundest argument, there is always room for doubt. Mart, bedankt voor je vriendschap, luisterend oor, en de prettige werksfeer die we samen hebben kunnen scheppen. lemand heeft ooit gezegd dat een slimmerik orde weet te houden, maar dat alleen een genie de chaos overziet. Wij weten allebei dat dat gewoon een mooi excuus is om er een rottroep van te maken op je buro. Ik hoop dat ik je nog minstens één keer mag inmaken met een potje Starcraft, voordat je weggaat uit Maastricht.

In fact, I feel fortunate with the whole environment I have worked in over the past years. The mythical 'Visiongroup', the Neurocognitie department, the Faculty of Psychology, concentric circles of people that work hard, are passionate about their work, but also sympathetic to the people they work with. I have especially enjoyed the interaction with the people in the inner circle, the Visiongroup. The conversations, discussions, parties, dinners, and mensa lunches at $13.15 \mathrm{~h}$ (did eventually get that through, didn't I?!) have greatly stimulated fun and enjoyment both at work and outside. Thanks to all of you, among others: Michael, Milene, Alex, the whole Italian gang (Federico, Giancarlo, Francesco, Fabrizio, and visiting: Luca and Angela), Amanda, Nienke, Vincent, Judith, Joel, Bettina, Rob, Peter, Sven, Pim, Vera, Teresa (I hate these lists, you always forget someone... Well, come by and beat me up over it...). I look forward to working in this stimulating environment a little longer.

Thanks to Erik (with a ' $k$ ') for enthousiastically helping with running and modifying DTI sequences for the past years. Thanks to Eric (with a 'c') for guiding me in my final student years and taking me under his wing as a student assistant. Thanks, Ralf and Oriana for the nice collaboration and your care in my short stay in Frankfurt. Barry and Fatima, thanks for the very pleasant and productive stay at the $\mathrm{NIH}$ around thanksgiving. 
Lieve $\mathrm{Pa}$ en $\mathrm{Ma}$, zonder jullie was ik er nooit geweest, maar dat is makkelijk. Zonder jullie was ik ook niet geweest wie ik nu ben. Mam, van jou heb ik geleerd om altijd, altijd, eerlijk en recht-door-zee te zijn. En om mijn ideeen altijd te verwezelijken, dat dromen werkelijkheid kunnen worden als je het echt wil. $\mathrm{Pa}$, van jou heb ik geleerd dat dat niet zomaar gaat, dat dromen realiseren veel werk, inzet en overgave vergt. Ik ben dankbaar voor alles waarin ik op jou lijk (zelfs die snel terug trekkende haargrens...). Van jullie allebei heb ik respect leren te hebben voor de mensen om me heen. Ik hoop dat ik nog lang van jullie mag blijven leren.

Thur, broerke, achteraf verbaast het me soms dat we onze 'fighting years' overleefd hebben, wat hebben we mekaar de koppen ingeslagen. Soms is het bizar om een halve kopie van mezelf te zien rond lopen die tegelijk zo hetzelfde en zo verschillend is, maar het is vooral fantastisch, ik hoop dat je mijn hele leven mijn beste broer blijft. Lieve lau, zusje, ik heb jou altijd als een voorbeeld in mijn leven gezien. En dat is niet alleen omdat je twee jaar ouder bent. Dat is om het geweldige gevoelige hardwerkende succesvolle persoon dat je bent. Ik prijs me gelukkig tussen jullie in geboren te zijn.

Jan en Michiel, gasten (boykes kan echt niet...), wie zou ik zijn zonder jullie vriendschap? Al jaren zijn we vrienden door dik en dun, komen we samen, wat er ook veranderd is de laatste tijd, maken we muziek (soms...), en praten over belangrijke dingen en onbelangrijke dingen met een fantastische humor die zijn weerga niet kent. We zijn in het verleden wel eens getypeerd als 'rather disturbed brothers in arms'. Ik zou die term hier niet willen gebruiken, maar ik hoop dat we in het bejaardentehuis nog steeds samen muziek maken.

Henk, jongen, onze programmeer avonden zijn me dierbaar geworden. Dat spel van ons komt er wel, al duurt het nog eens vier jaar (waarschijnlijk wel...). En anders hebben we op zijn minst een heleboel gezellig geouwehoerd. Jij en Bernie, bedankt voor de goede gesprekken in de kelder in de late uurtjes, onder het genot van een sigaretje (voor jullie dan...).

Mike, Selly, Nel, Ed, Anja, Albert, Vitòr-Jolie (hoop dat ik het goed geschreven heb, uitspreken gaat in ieder geval al beter, toch?), familie Vrancken en Dreezens, fijn dat jullie mijn tweede familie konden zijn, ik had het niet willen missen.

Lieve Ellen, een paar zinnen doen nauwelijks recht aan de steun, de vriendschap, de liefde, het geluk, alles dat ik van jou heb gekregen in de afgelopen jaren. leder ander woord in dit proefschrift, en in alle programmeercode die ervoor geschreven heb, zou jouw naam moeten zijn om zelfs maar in de buurt te komen. Ik heb het geprobeerd, maar de expositie boette nogal aan duidelijkheid in, en mijn compiler klaagde over 'undeclared identifiers'. Bedankt voor alles dat je me gegeven hebt en voor alles dat ik jou heb mogen geven. Er is altijd wel iemand, maar niemand als jij. 


\title{
Curriculum Vitae
}

\begin{abstract}
Alard Roebroeck was born on June 28, 1978, in Reuver. Although he didn't like tying his shoelaces in his younger years, and preferred Velcro shoes instead, he turned out alright. In 1996 he graduated from secondary school (Gymnasium, Bisschoppelijk college Broekhin in Roemond). From 1997 on, he studied Psychology and Knowledge Engineering at Maastricht University. He received his Propaeduese in Knowledge Engineering (cum laude) in 1999. Between 2000 and 2002, he was a research assistant at the Department of Computer Science, Maastricht University, implementing image recognition software. He graduated in Psychology (cum laude) in 2002, specializing in Neural modeling and Cognitive Neuroscience. In 2002 he began his PhD project at the Neurocognitie Department of the Faculty of Psychology, Maastricht University. Since September 2006, he is an assistant professor (universitair docent) at the Faculty of Psychology in Maastricht.
\end{abstract}

